\title{
Synthesis of 1,1-Diboryl Alkenes Using the Boryl-Heck Reaction
}

\author{
Olamide O. Idowu, Jacob C. Hayes, William B. Reid, and Donald A. Watson* \\ Department of Chemistry and Biochemistry, University of Delaware, \\ Newark, Delaware 19716, United States \\ dawatson@udel.edu
}

\section{Supporting Information}

Index

1. General Experimental Details: $\quad$ S2

2. Instrumentation and Chromatography: S2

3. Preparation of catBBr Solution in Toluene: $\quad$ S2

4. General Procedure for the Diborylation of Styrenes: S3

5. General Procedure for the Diborylation of a-Olefins S8

6. Decomposition Studies of catBBr $\quad \mathrm{S13}$

7. General Procedure for the Time Study Experiment $\quad$ S14

8. Studies Towards the Borylation of Trans-Monoboryl Alkenes S14

9. References $\quad$ S15

10. Spectral Data $\quad$ S15 


\section{General Experimental Details:}

Toluene was dried on alumina according to published procedures. ${ }^{1} B$-bromocatecholborane (catBBr) was purchased from $\mathrm{TCl}$. For preparative reactions, catBBr was stored as a toluene solution in a nitrogen-filled Straus flask at rt. $^{2}$ For reactions run in a glovebox (only), catBBr was stored as a solid in a nitrogen-filled glovebox freezer at $-35^{\circ} \mathrm{C}$. N,N-Dicyclohexylmethylamine was purchased from $\mathrm{TCl}$, distilled from calcium hydride $\left(80^{\circ} \mathrm{C}, 150 \mathrm{mtorr}\right)$ and stored at it in a nitrogen-filled Straus flask. Trifluorotoluene was purchased from Sigma Aldrich in an anhydrous septum sealed bottle, transferred to a Straus flask by cannula transfer, sparged with nitrogen for 15 minutes, and stored under nitrogen. Bis(3,5-di-tert-butylphenyl)(tert-butyl)phosphine (JessePhos $)^{3}$ and [(JessePhos)Pdl $\left.{ }_{2}\right]_{2}{ }^{4}$ were prepared according to published procedures. Ethyl 4-vinylbenzoate, ${ }^{5} \quad$ 2-vinylthiophene, ${ }^{6} \quad$ 1-(toluene-4-sulfonyl)2-vinyl-1 $\mathrm{H}$-indole, ${ }^{7} \quad{ }^{6}$ dimethylphenylsilylhex-1-ene, ${ }^{8} 7$-butoxyhept-1-ene, ${ }^{8}$ triisopropylsiloxy-5-hexene, ${ }^{8}$ and pivaloxy5 -hexene ${ }^{8}$ were prepared according to published literature procedures. All other substrates and reagents were purchased in highest analytical purity from commercial suppliers and used as received. All alkene substrates were sparged with nitrogen for 15 minutes before use. Vials used in the glovebox were dried in a gravity oven at $140{ }^{\circ} \mathrm{C}$ for a minimum of $12 \mathrm{~h}$, transferred into the glovebox hot, and then stored at $\mathrm{rt}$ in the glovebox prior to use. All other glassware was flamedried under vacuum prior to use. "Double manifold" refers to a standard Schlenk-line gas manifold equipped with nitrogen and vacuum (ca. $100 \mathrm{mtorr})$. All optimization reactions $(0.25 \mathrm{mmol})$ were run in a nitrogen-filled glovebox and heated using an aluminum block on a magnetic stir plate. All yields in optimization reactions were determined using ${ }^{1} \mathrm{H}$ NMR using 1,3,5-trimethoxybenzene as an internal standard and similarly, yields determined by GC analysis were obtained by using nonane as an internal standard. Decomposition reaction and time study reactions were run in a nitrogen-filled glovebox on a $0.25 \mathrm{mmol}$ scale $(0.1 \mathrm{M})$. All other reactions were set up using standard Schlenk technique and heated with stirring in temperature-controlled oil baths. Any product yields listed in the main text that do not match those listed in the supporting information are the average of multiple isolated yields. Note: The ${ }^{13} \mathrm{C}$ NMR signal for carbons attached to boron did not appear in the collected spectra due to the quadrupole splitting of ${ }^{11} \mathrm{~B} .{ }^{9}$

\section{Instrumentation and Chromatography:}

$400 \mathrm{MHz}{ }^{1} \mathrm{H}, 101 \mathrm{MHz}{ }^{13} \mathrm{C}$ and $128 \mathrm{MHz}{ }^{11} \mathrm{~B}$ spectra were obtained on a $400 \mathrm{MHz}$ FT-NMR spectrometer equipped with a Bruker CryoPlatform. $600 \mathrm{MHz}{ }^{1} \mathrm{H}, 151 \mathrm{MHz}{ }^{13} \mathrm{C}, 193 \mathrm{MHz}{ }^{11} \mathrm{~B}, 565$ $\mathrm{MHz}{ }^{19} \mathrm{~F}$, and $119 \mathrm{MHz}{ }^{29} \mathrm{Si}$ spectra were obtained on a $600 \mathrm{MHz}$ FT-NMR spectrometer equipped with a Bruker SMART probe. All samples were analyzed in the indicated deutero-solvent and were recorded at ambient temperatures. All chemical shifts are reported in ppm. ${ }^{1} \mathrm{H}$ NMR spectra were calibrated using the residual protio-signal in deutero-solvents as a standard. ${ }^{13} \mathrm{C} \mathrm{NMR}$ spectra were calibrated using the deutero-solvent signal as a standard. IR spectra were recorded on a Nicolet Magma-IR 560 FT-IR spectrometer as thin films on KBr plates. High resolution MS data was obtained on a Thermo Q-Exactive Orbitrap using electrospray ionization (ESI). Column chromatography was performed with boric acid impregnated 40-63 $\mu \mathrm{m}$ silica gel with the eluent reported in parentheses. Boric acid silica gel was prepared according to a known procedure. ${ }^{10}$ Analytical thin-layer chromatography (TLC) was performed on pre-coated glass plates and visualized by UV or by staining with $\left(\mathrm{KMnO}_{4}\right.$ or $\left.\mathrm{I}_{2}\right)$.

\section{Preparation of catBBr Solution in Toluene:}

A $25 \mathrm{~mL}$ Schlenk flask equipped with a magnetic stir bar and a rubber septum was flame-dried and cooled to rt under vacuum and then refilled with nitrogen. The flask was briefly opened, charged with $B$-bromocatecholborane $(1.59 \mathrm{~g}, 8 \mathrm{mmol})$, and resealed with a rubber septum. The 
flask was evacuated and refilled with nitrogen three times and left under positive nitrogen pressure. Anhydrous toluene $(10 \mathrm{~mL})$ was added via syringe and the solution was stirred under a nitrogen atmosphere until all the catBBr was dissolved. The solution was transferred into a nitrogen-filled $15 \mathrm{~mL}$ Straus flask by cannula transfer and stored at rt under a nitrogen atmosphere. The solution was titrated according to a previously described procedure. ${ }^{2}$

\section{General Procedure for the Diborylation of Styrenes:}

\section{General Procedure A (Styrenes):}

An oven dried $25 \mathrm{~mL}$ Schlenk-tube equipped with a magnetic stirbar and Teflon sealed valve was attached to a double manifold and cooled under vacuum. The flask was backfilled with nitrogen, briefly opened, charged with [(JessePhos) $\left.\mathrm{Pdl}_{2}\right]_{2}(41 \mathrm{mg}, 2.5 \mathrm{~mol} \%)$, and resealed. The flask was evacuated and refilled with nitrogen three times, and the Teflon valve was replaced with a rubber septum and left under a positive nitrogen pressure. Trifluorotoluene $(5 \mathrm{~mL})$ was added via syringe and the solution was stirred at rt for 10 minutes. $N, N$-dicyclohexylmethylamine $(1.1 \mathrm{~mL}, 5.0$ equiv), a solution of $B$-bromocatecholborane in toluene $(5 \mathrm{~mL}, \sim 0.8 \mathrm{M}, 4.0$ equiv), and alkene (1 mmol, 1.0 equiv) were added sequentially via syringe. The septum was replaced with a Teflon valve, the flask was sealed, and the reaction was stirred in an oil bath at $70{ }^{\circ} \mathrm{C}$ for $24 \mathrm{~h}$. At this time, the flask was removed from the oil bath and cooled to rt. The flask was opened to air, charged with pinacol (472 mg, 4.0 equiv), ammonium pyrrolidine-dithiocarbamate (50 mg, palladium scavenger, 6 equiv to palladium), ${ }^{11}$ and diluted with diethylether $(10 \mathrm{~mL})$. The reaction was stirred at $\mathrm{rt}$ for $1 \mathrm{~h}$. At this time, the reaction was filtered through a pad of Celite and concentrated in vacuo at $40{ }^{\circ} \mathrm{C}$ to remove all solvents. The resultant crude oil was diluted with $\mathrm{Et}_{2} \mathrm{O}(20 \mathrm{~mL})$ and washed with $1 \mathrm{M}$ aqueous $\mathrm{HCl}(3 \times 20 \mathrm{~mL})$ to remove excess amine. The organic layer was dried with $\mathrm{MgSO}_{4}$, filtered through Celite, and concentrated in vacuo. The crude material was purified via column chromatography using boric acid impregnated silica gel ${ }^{10}$ in the indicated solvent system.<smiles>CC(C)(C)c1ccc(C=C(Br)c2ccccc2)cc1</smiles>

(3) According to General Procedure A, [(JessePhos)Pdl $]_{2}$ (41 mg, 2.5 $\mathrm{mol} \%)$, trifluorotoluene $(5 \mathrm{~mL}, 0.1 \mathrm{M}), N, N$-dicyclohexylmethylamine $(1.1$ $\mathrm{mL}, 5.0 \mathrm{mmol})$, catBBr $(5 \mathrm{~mL}, 4.0 \mathrm{mmol})$ and 4-tertbutyl styrene $(183 \mu \mathrm{L}$, $1.0 \mathrm{mmol}$ ) were combined under $\mathrm{N}_{2}$ and stirred at $70{ }^{\circ} \mathrm{C}$ for $24 \mathrm{~h}$. The reaction was worked up according to the General Procedure. The product was purified on boric acid impregnated silica gel $(50 \mathrm{~mL}, 1: 9$ diethyl ether : hexanes) to afford 3 as a colorless non-crystalline solid (384 mg, 93\%). Note: The average yield for this compound over two runs was $\mathbf{9 3} \%$, which is the yield reported in the main text.

${ }^{1} \mathrm{H}$ NMR $\left(600 \mathrm{MHz}, \mathrm{CDCl}_{3}\right) \delta 7.68(\mathrm{~s}, 1 \mathrm{H}), 7.42(\mathrm{~d}, J=8.0 \mathrm{~Hz}, 2 \mathrm{H}), 7.30(\mathrm{~d}, J=8.5 \mathrm{~Hz}, 2 \mathrm{H}), 1.33$ $(\mathrm{s}, 12 \mathrm{H}), 1.30(\mathrm{~s}, 9 \mathrm{H}), 1.28(\mathrm{~s}, 12 \mathrm{H})$.

${ }^{13} \mathrm{C}$ NMR $\left(151 \mathrm{MHz}, \mathrm{CDCl}_{3}\right) \delta 155.2,151.8,136.9,128.2,125.2,83.7,83.3,34.8,31.4,25.0,24.8$.

${ }^{11} \mathrm{~B} \mathrm{NMR}\left(193 \mathrm{MHz}, \mathrm{CDCl}_{3}\right) \delta 31.1$.

FTIR $\left(\mathrm{cm}^{-1}\right)$ 2976, 1604, 1334, 1142, 858.

HRMS (ESI) m/z: [M + H] + Calcd for $\mathrm{C}_{24} \mathrm{H}_{39} \mathrm{~B}_{2} \mathrm{O}_{4}$ 413.3029; Found 413.3016. Found 
<smiles>C/C(Br)=C/c1ccc(C)cc1C</smiles>

(4) According to General Procedure A, [(JessePhos)Pdl $]_{2}$ (41 mg, 2.5 $\mathrm{mol} \%)$, trifluorotoluene $(5 \mathrm{~mL}, 0.1 \mathrm{M}), N, N$-dicyclohexylmethylamine $(1.1$ $\mathrm{mL}, 5.0 \mathrm{mmol})$, catBBr $(5 \mathrm{~mL}, 4.0 \mathrm{mmol})$ and 2,4-dimethylstyrene (146 $\mu \mathrm{L}, 1.0 \mathrm{mmol}$ ) were combined under $\mathrm{N}_{2}$ and stirred at $70^{\circ} \mathrm{C}$ for $24 \mathrm{~h}$. The reaction was worked up according to the General Procedure. The product was purified on boric acid impregnated silica gel $(50 \mathrm{~mL}, 1$ : 99 ethyl acetate : hexanes) to afford 4 as a faint yellow solid (344 mg, 89\%).

${ }^{1} \mathrm{H} \mathrm{NMR}\left(600 \mathrm{MHz}, \mathrm{CDCl}_{3}\right) \delta 7.87(\mathrm{~s}, 1 \mathrm{H}), 7.39(\mathrm{~d}, J=7.7 \mathrm{~Hz}, 1 \mathrm{H}), 6.92(\mathrm{~d}, J=10.4 \mathrm{~Hz}, 2 \mathrm{H}), 2.33$ (s, 3H), $2.29(\mathrm{~s}, 3 \mathrm{H}), 1.28(\mathrm{~s}, 12 \mathrm{H}), 1.26(\mathrm{~s}, 12 \mathrm{H})$.

${ }^{13} \mathrm{C}$ NMR $\left(151 \mathrm{MHz}, \mathrm{CDCl}_{3}\right) \delta 154.0,138.2,136.4,136.3,130.8,127.7,126.3,83.5,83.1,25.0$, 24.7, 21.3, 19.8 .

${ }^{11} \mathrm{~B}$ NMR $\left(193 \mathrm{MHz}, \mathrm{CDCl}_{3}\right) \delta 30.0$.

FTIR $\left(\mathrm{cm}^{-1}\right)$ 2978, 2929, 1598, 1566, 1497, 1371, 1332, 1268, 1235, 1213, 1142, 1110, 1011, 990, 966.

m.p. $=90-94^{\circ} \mathrm{C}$.

HRMS (ESI) m/z: [M + H]+ Calcd for $\mathrm{C}_{22} \mathrm{H}_{35} \mathrm{~B}_{2} \mathrm{O}_{4}$ 385.2721; Found 385.2719.Found<smiles>COc1ccc(/C=C(\[B]c2ccccc2)c2ccccc2)cc1</smiles>

(5) According to General Procedure A, [(JessePhos)Pdl $]_{2}$ (41 mg, 2.5 mol \%), trifluorotoluene $(5 \mathrm{~mL}, 0.1 \mathrm{M}), \mathrm{N}, \mathrm{N}$-dicyclohexylmethylamine $(1.1 \mathrm{~mL}, 5.0 \mathrm{mmol})$, catBBr $(5 \mathrm{~mL}, 4.0 \mathrm{mmol})$ and alkene $(133 \mu \mathrm{L}, 1.0$ mmol) were combined under $\mathrm{N}_{2}$ and stirred at $70{ }^{\circ} \mathrm{C}$ for $24 \mathrm{~h}$. The reaction was worked up according to the General Procedure. The product was purified on boric acid impregnated silica gel $(50 \mathrm{~mL}, 1: 9$ dichloromethane : hexanes) to afford a 5 as a black solid (343 $\mathrm{mg}, 89 \%$ ).

${ }^{1} \mathrm{H}$ NMR $\left(600 \mathrm{MHz}, \mathrm{CDCl}_{3}\right) \delta 7.65(\mathrm{~s}, 1 \mathrm{H}), 7.44(\mathrm{~d}, J=8.7 \mathrm{~Hz}, 2 \mathrm{H}), 6.82(\mathrm{~d}, J=8.7 \mathrm{~Hz}, 2 \mathrm{H}), 3.80$ $(\mathrm{s}, 3 \mathrm{H}), 1.32(\mathrm{~s}, 12 \mathrm{H}), 1.27(\mathrm{~s}, 12 \mathrm{H})$.

${ }^{13} \mathrm{C}$ NMR $\left(151 \mathrm{MHz}, \mathrm{CDCl}_{3}\right) \delta$ 160.1, 154.9, 132.7, 129.9, 113.7, 83.7, 83.2, 55.4, 25.0, 24.8.

${ }^{11} \mathrm{~B}$ NMR $\left(193 \mathrm{MHz}, \mathrm{CDCl}_{3}\right) \delta 30.3$.

FTIR $\left(\mathrm{cm}^{-1}\right)$ 2977, 2932, 2837, 1604, 1511, 1336, 1141, 1032, 857.

m.p. $=80.5-82^{\circ} \mathrm{C}$.

HRMS (ESI) m/z: [M + H]+ Calcd for $\mathrm{C}_{21} \mathrm{H}_{33} \mathrm{~B}_{2} \mathrm{O}_{5}$ 387.2514; Found 387.2512.<smiles>CN(C)c1ccc(/C=C(\[Pb])c2ccccc2)cc1</smiles>

(6) A modified version of General Procedure A was used. [(JessePhos) $\left.\mathrm{Pdl}_{2}\right]_{2}(41 \mathrm{mg}, 2.5 \mathrm{~mol} \%)$, trifluorotoluene $(4 \mathrm{~mL}, 0.1 \mathrm{M})$, $N, N$-dicyclohexylmethylamine $(1.1 \mathrm{~mL}, 5.0 \mathrm{mmol})$, catBBr $(5 \mathrm{~mL}, 4.0$ mmol) were combined under $\mathrm{N}_{2}$ and stirred at $70^{\circ} \mathrm{C}$. A solution of alkene ( $147 \mathrm{mg}, 1.0 \mathrm{mmol}$ ) in trifluorotoluene $(1 \mathrm{~mL})$ was added slowly over 2 hours via syringe pump. Once the addition was complete, the septum was replaced with a Teflon valve \& stirred for $22 \mathrm{~h}$. At this time, the flask was removed from the oil bath and cooled to rt. The flask was opened 
to air, charged with pinacol (472 mg, 4.0 equiv), ammonium pyrrolidine-dithiocarbamate (50 mg, palladium scavenger, 6 equiv to palladium), and diluted with hexanes $(10 \mathrm{~mL})$. The reaction was stirred at rt for $1 \mathrm{~h}$. At that time, the reaction was filtered through a pad of Celite and concentrated in vacuo at $40{ }^{\circ} \mathrm{C}$ to remove all solvents. The crude oil was purified by column chromatography on boric acid impregnated silica gel ( $75 \mathrm{~mL}, 3: 97$ ethyl acetate : hexanes) to afford 6 as a yellow oil. (290 mg, 73\%)

${ }^{1} \mathrm{H}$ NMR $\left(600 \mathrm{MHz}, \mathrm{CDCl}_{3}\right) \delta 7.62(\mathrm{~s}, 1 \mathrm{H}), 7.41(\mathrm{~d}, J=8.8 \mathrm{~Hz}, 2 \mathrm{H}), 6.61(\mathrm{~d}, J=8.9 \mathrm{~Hz}, 2 \mathrm{H}), 2.96$ $(\mathrm{s}, 6 \mathrm{H}), 1.34(\mathrm{~s}, 12 \mathrm{H}), 1.26(\mathrm{~s}, 12 \mathrm{H})$.

${ }^{13} \mathrm{C}$ NMR $\left(151 \mathrm{MHz}, \mathrm{CDCl}_{3}\right) \delta 155.7,150.8,129.9,128.1,111.7,83.5,83.0,40.4,25.0,24.9$.

${ }^{11} \mathrm{~B}$ NMR $\left(193 \mathrm{MHz}, \mathrm{CDCl}_{3}\right) \delta 30.4$.

FTIR $\left(\mathrm{cm}^{-1}\right)$ 2978, 2930, 2807, 1592, 1522, 1390, 1335, 1222, 1187, 1143, 1010, 989.

HRMS (ESI) m/z: [M + H]+ Calcd for $\mathrm{C}_{22} \mathrm{H}_{36} \mathrm{~B}_{2} \mathrm{NO}_{4}$ 400.2830; Found 400.2825.<smiles>FC(F)(F)c1ccc(/C=C(\[Pb])c2ccccc2)cc1</smiles>

worked up according to whe General Procedure. The product was purified on boric acid impregnated silica gel ( $50 \mathrm{~mL}, 2: 98$ ethyl acetate : hexanes) to afford 7 as a white solid (394 mg, $93 \%)$.

${ }^{1} \mathrm{H}$ NMR (600 MHz, $\left.\mathrm{CDCl}_{3}\right) \delta 7.70(\mathrm{~s}, 1 \mathrm{H}), 7.56(\mathrm{q}, \mathrm{J}=8.3 \mathrm{~Hz}, 4 \mathrm{H}), 1.31(\mathrm{~s}, 12 \mathrm{H}), 1.29(\mathrm{~s}, 12 \mathrm{H})$.

${ }^{13} \mathrm{C}$ NMR $\left(151 \mathrm{MHz}, \mathrm{CDCl}_{3}\right) \delta 153.2,143.1,130.2$ (q, $\left.J=32.4 \mathrm{~Hz}\right), 128.4,125.3$ (q, $\left.J=3.7 \mathrm{~Hz}\right)$, 123.4, 84.0, 83.6, 25.0, 24.8.

${ }^{11} \mathrm{~B}$ NMR $\left(193 \mathrm{MHz}, \mathrm{CDCl}_{3}\right) \delta 30.0$.

${ }^{19} \mathrm{~F}$ NMR $\left(565 \mathrm{MHz}, \mathrm{CDCl}_{3}\right) \delta-62.6$.

FTIR $\left(\mathrm{cm}^{-1}\right)$ 2979, 2932, 1610, 1573, 1390, 1323, 1212, 1140, 1067, 856.

m.p. $=86-88^{\circ} \mathrm{C}$.

HRMS (ESI) m/z: [M + H]+ Calcd for $\mathrm{C}_{21} \mathrm{H}_{30} \mathrm{~B}_{2} \mathrm{O}_{4} \mathrm{~F}_{3}$ 425.2282; Found 425.2284.<smiles>CCOC(=O)c1ccc(C=C([B]c2ccccc2)[B]c2ccccc2)cc1</smiles>

(8) According to General Procedure A, [(JessePhos)Pdl $]_{2}$ (41 mg, 2.5 mol \%), trifluorotoluene $(5 \mathrm{~mL}, 0.1 \mathrm{M}), \mathrm{N}, \mathrm{N}$-dicyclohexylmethylamine $(1.1 \mathrm{~mL}, 5.0 \mathrm{mmol})$, catBBr $(5 \mathrm{~mL}, 4.0 \mathrm{mmol})$ and alkene $(168 \mu \mathrm{L}, 1.0$ mmol) were combined under $\mathrm{N}_{2}$ and stirred at $70{ }^{\circ} \mathrm{C}$ for $24 \mathrm{~h}$. The reaction was worked up according to the General Procedure. The product was purified on boric acid impregnated silica gel (50 mL, 2 : 98 ethyl acetate : hexanes) to afford 8 as a pale yellow solid (372 $\mathrm{mg}, 73 \%$ ).

${ }^{1} \mathrm{H}$ NMR $\left(600 \mathrm{MHz}, \mathrm{CDCl}_{3}\right) \delta 7.97(\mathrm{~d}, J=8.3 \mathrm{~Hz}, 2 \mathrm{H}), 7.71(\mathrm{~s}, 1 \mathrm{H}), 7.54(\mathrm{~d}, J=8.3 \mathrm{~Hz}, 2 \mathrm{H}), 4.36$ (q, $J=7.0 \mathrm{~Hz}, 2 \mathrm{H}), 1.39(\mathrm{t}, J=7.1 \mathrm{~Hz}, 3 \mathrm{H}), 1.31(\mathrm{~s}, 12 \mathrm{H}), 1.28(\mathrm{~s}, 12 \mathrm{H})$. 
${ }^{13} \mathrm{C}$ NMR $\left(151 \mathrm{MHz}, \mathrm{CDCl}_{3}\right) \delta 166.6,153.8,144.0,130.2,129.6,128.1,84.0,83.6,61.1,25.0$, $24.8,14.5$.

${ }^{11} \mathrm{~B}$ NMR $\left(193 \mathrm{MHz}, \mathrm{CDCl}_{3}\right) \delta 31.1$.

FTIR $\left(\mathrm{cm}^{-1}\right)$ 2978, 2931, 1721, 1605, 1565, 1334, 1272, 1140, 1107, 1011, 852.

m.p. $=121-122.5^{\circ} \mathrm{C}$.

HRMS (ESI) m/z: [M + H]+ Calcd for $\mathrm{C}_{23} \mathrm{H}_{35} \mathrm{~B}_{2} \mathrm{O}_{6}$ 429.2619; Found 429.2622.<smiles></smiles>

10

(10) According to General Procedure A, [(JessePhos)Pdl $]_{2}(41 \mathrm{mg}, 2.5 \mathrm{~mol}$ $\%)$, trifluorotoluene $(5 \mathrm{~mL}, 0.1 \mathrm{M}), N, N$-dicyclohexylmethylamine $(1.1 \mathrm{~mL}$, $5.0 \mathrm{mmol})$, catBBr $(5 \mathrm{~mL}, 4.0 \mathrm{mmol})$ and alkene $(119 \mu \mathrm{L}, 1.0 \mathrm{mmol})$ were combined under $\mathrm{N}_{2}$ and stirred at $70{ }^{\circ} \mathrm{C}$ for $24 \mathrm{~h}$. The reaction was worked up according to the General Procedure. The product was purified on boric acid impregnated silica gel $(50 \mathrm{~mL}, 2: 98$ then 5 : 95 ethyl acetate : hexanes) to afford 10 as a white solid (283 $\mathrm{mg}, 76 \%)$.

${ }^{1} \mathrm{H}$ NMR $\left(600 \mathrm{MHz}, \mathrm{CDCl}_{3}\right) \delta 7.66(\mathrm{~s}, 1 \mathrm{H}), 7.49-7.43(\mathrm{~m}, 2 \mathrm{H}), 7.01-6.95(\mathrm{~m}, 2 \mathrm{H}), 1.31(\mathrm{~s}, 12 \mathrm{H})$, $1.27(\mathrm{~s}, 12 \mathrm{H})$.

${ }^{13} \mathrm{C} \mathrm{NMR}\left(151 \mathrm{MHz}, \mathrm{CDCl}_{3}\right) \delta 163.0(\mathrm{~d}, J=248.0 \mathrm{~Hz}), 153.9,136.0(\mathrm{~d}, J=3.4 \mathrm{~Hz}), 130.1$ (d, $J=$ $7.9 \mathrm{~Hz}), 115.2(\mathrm{~d}, \mathrm{~J}=21.7 \mathrm{~Hz}), 83.8,83.4,25.0,24.8$.

${ }^{11} \mathrm{~B}$ NMR $\left(193 \mathrm{MHz}, \mathrm{CDCl}_{3}\right) \delta 30.3$.

${ }^{19} \mathrm{~F} \mathrm{NMR}\left(565 \mathrm{MHz}, \mathrm{CDCl}_{3}\right) \delta-113.1$.

FTIR $\left(\mathrm{cm}^{-1}\right)$ 2979, 2931, 1602, 1587, 1508, 1389, 1334, 1308, 1256, 1141, 1011, 858.

m.p. $=94.5-97^{\circ} \mathrm{C}$.

HRMS (ESI) m/z: [M + H] + Calcd for $\mathrm{C}_{20} \mathrm{H}_{30} \mathrm{~B}_{2} \mathrm{O}_{4} \mathrm{~F}$ 375.2314; Found 375.2314.<smiles></smiles>

11

(11) According to General Procedure A, [(JessePhos)Pdl $]_{2}$ (41 mg, 2.5 $\mathrm{mol} \%)$, trifluorotoluene $(5 \mathrm{~mL}, 0.1 \mathrm{M}), N, N$-dicyclohexylmethylamine $(1.1$ $\mathrm{mL}, 5.0 \mathrm{mmol})$, catBBr $(5 \mathrm{~mL}, 4.0 \mathrm{mmol})$ and alkene $(120 \mu \mathrm{L}, 1.0 \mathrm{mmol})$ were combined under $\mathrm{N}_{2}$ and stirred at $70{ }^{\circ} \mathrm{C}$ for $24 \mathrm{~h}$. The reaction was worked up according to the General Procedure. The product was purified on boric acid impregnated silica gel $(50 \mathrm{~mL}, 1: 100$ ethyl acetate : hexanes) to afford 11 as a white solid (341 mg, 87\%).

${ }^{1} \mathrm{H}$ NMR $\left(600 \mathrm{MHz}, \mathrm{CDCl}_{3}\right) \delta 7.64(\mathrm{~s}, 1 \mathrm{H}), 7.42(\mathrm{~d}, J=8.5 \mathrm{~Hz}, 2 \mathrm{H}), 7.27(\mathrm{~d}, J=5.6 \mathrm{~Hz}, 2 \mathrm{H}), 1.31$ (s, 12H), $1.28(\mathrm{~s}, 12 \mathrm{H})$.

${ }^{13} \mathrm{C}$ NMR $\left(151 \mathrm{MHz}, \mathrm{CDCl}_{3}\right) \delta 153.7,138.3,134.4,129.6,128.5,83.9,83.5,25.0,24.8$.

${ }^{11} \mathrm{~B}$ NMR $\left(193 \mathrm{MHz}, \mathrm{CDCl}_{3}\right) \delta 30.5$.

FTIR $\left(\mathrm{cm}^{-1}\right)$ 2978, 2931, 1605, 1490, 1333, 1253, 1140, 1012, 856. 
m.p. $=85-86^{\circ} \mathrm{C}$.

HRMS (ESI) m/z: [M + H]+ Calcd for $\mathrm{C}_{20} \mathrm{H}_{30} \mathrm{~B}_{2} \mathrm{O}_{4} \mathrm{Cl}$ 391.2018; Found 391.2021.<smiles>Brc1ccc(/C=C(\[Pb])c2ccccc2)cc1</smiles>

12

(12) According to General Procedure A, [(JessePhos)Pdl $]_{2}$ (41 mg, 2.5 mol \%), trifluorotoluene $(5 \mathrm{~mL}, 0.1 \mathrm{M}), N, N$-dicyclohexylmethylamine $(1.1$ $\mathrm{mL}, 5.0 \mathrm{mmol})$, catBBr $(5 \mathrm{~mL}, 4.0 \mathrm{mmol})$ and alkene $(131 \mu \mathrm{L}, 1.0 \mathrm{mmol})$ were combined under $\mathrm{N}_{2}$ and stirred at $70{ }^{\circ} \mathrm{C}$ for $24 \mathrm{~h}$. The reaction was worked up according to the General Procedure. The product was purified on boric acid impregnated silica gel (50 mL, $1: 100$ ethyl acetate : hexanes) to afford 12 as a white solid (201 mg, 47\%).

${ }^{1} \mathrm{H}$ NMR $\left(600 \mathrm{MHz}, \mathrm{CDCl}_{3}\right) \delta 7.62(\mathrm{~s}, 1 \mathrm{H}), 7.42(\mathrm{~d}, J=8.5 \mathrm{~Hz}, 2 \mathrm{H}), 7.35(\mathrm{~d}, J=8.5 \mathrm{~Hz}, 2 \mathrm{H}), 1.31$ (s, 12H), $1.27(\mathrm{~s}, 12 \mathrm{H})$.

${ }^{13} \mathrm{C}$ NMR $\left(151 \mathrm{MHz}, \mathrm{CDCl}_{3}\right) \delta 153.7,138.7,131.4,129.9,122.7,83.9,83.5,25.0,24.8$.

${ }^{11} \mathrm{~B}$ NMR $\left(193 \mathrm{MHz}, \mathrm{CDCl}_{3}\right) \delta 30.3$

FTIR $\left(\mathrm{cm}^{-1}\right)$ 2977, 2929, 1605, 1486, 1333, 1253, 1140, 1010, 856.

m.p. $=89-92{ }^{\circ} \mathrm{C}$.

HRMS (ESI) m/z [M + H] + Calcd for $\mathrm{C}_{20} \mathrm{H}_{30} \mathrm{~B}_{2} \mathrm{O}_{4} \mathrm{Br}$ 435.1514; Found 435.1528.

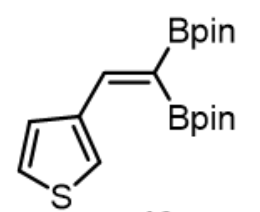

13

(13) According to General Procedure A, [(JessePhos)Pdl $]_{2}$ (41 mg, $2.5 \mathrm{~mol} \%$, trifluorotoluene $(5 \mathrm{~mL}, 0.1 \mathrm{M}), N, N$-dicyclohexylmethylamine $(1.1 \mathrm{~mL}, 5.0 \mathrm{mmol})$, catBBr $(5 \mathrm{~mL}, 4.0 \mathrm{mmol})$ and alkene $(102 \mu \mathrm{L}, 1.0 \mathrm{mmol})$ were combined under $\mathrm{N}_{2}$ and stirred at $70{ }^{\circ} \mathrm{C}$ for $24 \mathrm{~h}$. The reaction was worked up according to the General Procedure. The product was purified on boric acid impregnated silica gel $(50 \mathrm{~mL}$, $1: 100$ ethyl acetate : hexanes) to afford 13 as a white solid (257 mg, $71 \%)$. Note:

The average yield for this compound over two runs was $71 \%$, which is the yield reported in the main text.

${ }^{1} \mathrm{H}$ NMR $\left(600 \mathrm{MHz}, \mathrm{CDCl}_{3}\right) \delta 7.64(\mathrm{~s}, 1 \mathrm{H}), 7.44(\mathrm{~m}, 1 \mathrm{H}), 7.31$ (dd, J=5.0, $\left.1.3 \mathrm{~Hz}, 1 \mathrm{H}\right), 7.22$ (dd, $J=5.0,2.9 \mathrm{~Hz}, 1 \mathrm{H}), 1.33(\mathrm{~s}, 12 \mathrm{H}), 1.27(\mathrm{~s}, 12 \mathrm{H})$.

${ }^{13} \mathrm{C}$ NMR $\left(151 \mathrm{MHz}, \mathrm{CDCl}_{3}\right) \delta$ 148.3, 142.7, 127.4, 125.8, 125.5, 83.8, 83.3, 25.0, 24.9.

${ }^{11} \mathrm{~B}$ NMR $\left(193 \mathrm{MHz}, \mathrm{CDCl}_{3}\right) \delta 32.6$.

FTIR $\left(\mathrm{cm}^{-1}\right)$ 2977, 2929, 1600, 1321, 1263, 1140, 1011, 971, 854.

m.p. $=63-65^{\circ} \mathrm{C}$.

HRMS (ESI) m/z: [M + H]+ Calcd for $\mathrm{C}_{18} \mathrm{H}_{29} \mathrm{~B}_{2} \mathrm{O}_{4} \mathrm{~S}$ 363.1973; Found 363.1972. 
(14) According to General Procedure A, [(JessePhos)Pdl $]_{2}$ (41 mg, $2.5 \mathrm{~mol}$

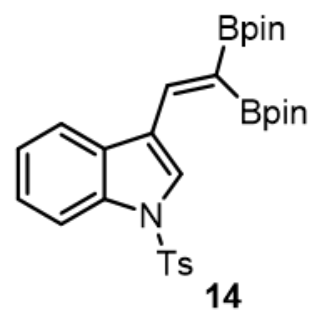

$\%)$, trifluorotoluene $(5 \mathrm{~mL}, 0.1 \mathrm{M}), N, N$-dicyclohexylmethylamine $(1.1 \mathrm{~mL}, 5.0$ $\mathrm{mmol})$, catBBr $(5 \mathrm{~mL}, 4.0 \mathrm{mmol})$ and alkene $(296 \mathrm{mg}, 1.0 \mathrm{mmol})$ were combined under $\mathrm{N}_{2}$ and stirred at $70{ }^{\circ} \mathrm{C}$ for $24 \mathrm{~h}$. The reaction was worked up according to the General Procedure. The product was purified on boric acid impregnated silica gel ( $50 \mathrm{~mL}, 5: 95$ ethyl acetate : hexanes) to afford 14 as a pale yellow solid (341 $\mathrm{mg}, 62 \%)$.

${ }^{1} \mathrm{H}$ NMR $\left(600 \mathrm{MHz}, \mathrm{CDCl}_{3}\right) \delta 8.11(\mathrm{~s}, 1 \mathrm{H}), 7.93(\mathrm{~d}, \mathrm{~J}=8.3 \mathrm{~Hz}, 1 \mathrm{H}), 7.74(\mathrm{~s}$, $1 \mathrm{H}), 7.73(\mathrm{~d}, J=8.2 \mathrm{~Hz}, 2 \mathrm{H}), 7.67(\mathrm{~d}, J=7.8 \mathrm{~Hz}, 1 \mathrm{H}), 7.32-7.26(\mathrm{~m}, 1 \mathrm{H}), 7.25-7.21(\mathrm{~m}, 1 \mathrm{H})$, $7.19(\mathrm{~d}, \mathrm{~J}=8.1 \mathrm{~Hz}, 2 \mathrm{H}), 2.32(\mathrm{~s}, 3 \mathrm{H}), 1.37(\mathrm{~s}, 13 \mathrm{H}), 1.28(\mathrm{~s}, 13 \mathrm{H})$.

${ }^{13} \mathrm{C}$ NMR $\left(151 \mathrm{MHz}, \mathrm{CDCl}_{3}\right) \delta 145.1,143.7,135.3,135.0,130.4,127.0,124.9,123.5,122.8$, 120.0, 113.6, 84.0, 83.4, 25.0, 25.0, 21.7.

${ }^{11} \mathrm{~B}$ NMR $\left(193 \mathrm{MHz}, \mathrm{CDCl}_{3}\right) \delta 31.1$.

FTIR $\left(\mathrm{cm}^{-1}\right)$ 3145, 2977, 2930, 1597, 1446, 1372, 1351, 1306, 1174, 1139, 1012, 971, 855.

m.p. $=195-196{ }^{\circ} \mathrm{C}$.

HRMS (ESI) m/z: [M + H] + Calcd for $\mathrm{C}_{29} \mathrm{H}_{38} \mathrm{~B}_{2} \mathrm{O}_{6} \mathrm{NS} 550.2605$; Found 550.2612.

\section{General Procedure for the Diborylation of $\alpha$-Olefins:}

\section{General Procedure B ( $\alpha$-Olefins):}

An oven dried $25 \mathrm{~mL}$ Schlenk tube equipped with a magnetic stirbar and Teflon-sealed valve was attached to a double manifold and cooled under vacuum. The flask was backfilled with nitrogen, briefly opened, charged with [(JessePhos) $\left.\mathrm{Pdl}_{2}\right]_{2}(82 \mathrm{mg}, 5 \mathrm{~mol} \%)$, and resealed. The flask was evacuated and refilled with nitrogen three times, and the Teflon valve was replaced with a rubber septum and left under a positive nitrogen pressure. Trifluorotoluene $(5 \mathrm{~mL})$ was added via syringe and the solution was stirred at $\mathrm{rt}$ for 10 minutes. $N, N$-dicyclohexylmethylamine $(1.1 \mathrm{~mL}, 5.0$ equiv), a solution of $B$-bromocatecholborane in toluene $(5 \mathrm{~mL}, \sim 0.8 \mathrm{M}, 4.0$ equiv), and alkene (1 mmol, 1.0 equiv) were added sequentially via syringe. The septum was replaced with a Teflon valve, the flask was sealed, and the reaction was stirred in an oil bath at $70{ }^{\circ} \mathrm{C}$ for $24 \mathrm{~h}$. At this time, the flask was removed from the oil bath and cooled to rt. The flask was opened to air, charged with pinacol (472 mg, 4.0 equiv), ammonium pyrrolidine-dithiocarbamate (50 mg, palladium scavenger, 6 equiv to palladium), ${ }^{10}$ and diluted with hexanes $(10 \mathrm{~mL})$. The reaction was stirred at $\mathrm{rt}$ for $1 \mathrm{~h}$. At this time, the reaction was filtered through a pad of Celite and concentrated in vacuo at $40{ }^{\circ} \mathrm{C}$ to remove all solvents. The resultant crude oil was diluted with hexanes $(20 \mathrm{~mL})$ and washed with $1 \mathrm{M}$ aqueous $\mathrm{HCl}(3 \times 20 \mathrm{~mL})$ to remove excess amine. The organic layer was dried with $\mathrm{MgSO}_{4}$, filtered through Celite, and concentrated in vacuo. The crude material was purified via column chromatography using boric acid impregnated silica ge ${ }^{10}$ in the indicated solvent system. Note: Purifying these compounds immediately after workup results in an inseparable, unidentified impurity at $6.40 \mathrm{ppm}$ (triplet, $0.05 \mathrm{H}$ ) in the purified product. We found that this impurity diminishes over time and is absent after $\sim 12 \mathrm{~h}$. Thus, after workup, the crude product was placed in a refrigerator overnight \& purified the next day. 


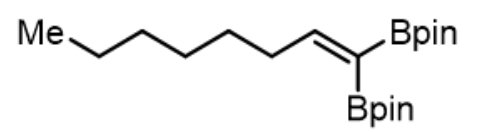

15

(15) According to General Procedure B, [(JessePhos)Pdl $]_{2}$ (82 mg, $5.0 \mathrm{~mol} \%)$, trifluorotoluene $(5 \mathrm{~mL}, \quad 0.1 \mathrm{M}), \quad \mathrm{N}, \mathrm{N}-$ dicyclohexylmethylamine $(1.1 \mathrm{~mL}, 5.0 \mathrm{mmol})$, catBBr $(5 \mathrm{~mL}, 4.0$ $\mathrm{mmol})$ and alkene $(156 \mu \mathrm{L}, 1.0 \mathrm{mmol})$ were combined under $\mathrm{N}_{2}$ and stirred at $70{ }^{\circ} \mathrm{C}$ for $24 \mathrm{~h}$. The reaction was worked up according to the General Procedure. The product was purified on boric acid impregnated silica gel (30 mL, $1: 9$ then $3: 7$ then $1: 1$ dichloromethane : hexanes) to afford 15 as a colorless oil (258 $\mathrm{mg}, 71 \%)$. Note: The average yield for this compound over two runs was $72 \%$, which is the yield reported in the main text. ${ }^{12}$

${ }^{1} \mathrm{H} \mathrm{NMR}\left(600 \mathrm{MHz}, \mathrm{CDCl}_{3}\right) \delta 6.93(\mathrm{t}, J=7.2 \mathrm{~Hz}, 1 \mathrm{H}), 2.25$ (q, $J=7.4 \mathrm{~Hz}, 2 \mathrm{H}$ ), 1.41 (ddd, $J=15.1$, 8.0, 6.4 Hz, 2H), $1.29(\mathrm{~s}, 12 \mathrm{H}), 1.28-1.24(\mathrm{~m}, 6 \mathrm{H}), 1.23(\mathrm{~s}, 12 \mathrm{H}), 0.87(\mathrm{t}, J=6.9 \mathrm{~Hz}, 3 \mathrm{H})$.

${ }^{13} \mathrm{C}$ NMR $\left(151 \mathrm{MHz}, \mathrm{CDCl}_{3}\right) \delta$ 162.7, 83.2, 82.9, 35.6, 31.8, 29.2, 29.1, 25.0, 24.9, 22.7, 14.2.

${ }^{11} \mathrm{~B}$ NMR $\left(193 \mathrm{MHz}, \mathrm{CDCl}_{3}\right) \delta 30.3$.

FTIR $\left(\mathrm{cm}^{-1}\right)$ 2977, 2927, 2856, 1609, 1467, 1343, 1144, 1009, 854.

HRMS (ESI) m/z: [M + H]+ Calcd for $\mathrm{C}_{20} \mathrm{H}_{39} \mathrm{~B}_{2} \mathrm{O}_{4}$ 365.3035; Found 365.3034.<smiles>BrC(=CCCc1ccccc1)c1ccccc1</smiles>

16

(16) According to General Procedure B, [(JessePhos)Pdl $]_{2}$ (82 mg, 5.0 $\mathrm{mol} \%)$, trifluorotoluene $(5 \mathrm{~mL}, 0.1 \mathrm{M}), N, N$-dicyclohexylmethylamine $(1.1$ $\mathrm{mL}, 5.0 \mathrm{mmol})$, catBBr $(5 \mathrm{~mL}, 4.0 \mathrm{mmol})$ and alkene $(150 \mu \mathrm{L}, 1.0 \mathrm{mmol})$ were combined under $\mathrm{N}_{2}$ and stirred at $70{ }^{\circ} \mathrm{C}$ for $24 \mathrm{~h}$. The reaction was worked up according to the General Procedure. The product was purified on boric acid impregnated silica gel (30 mL, $1: 100$ ethyl acetate : hexanes) to afford 16 as a colorless oil (261 $\mathrm{mg}, 68 \%$ ). Note: The average yield for this compound over two runs was $68 \%$, which is the yield reported in the main text.

${ }^{1} \mathrm{H}$ NMR $\left(400 \mathrm{MHz}, \mathrm{CDCl}_{3}\right) \delta 7.28-7.25(\mathrm{~m}, 2 \mathrm{H}), 7.20-7.17(\mathrm{~m}, 3 \mathrm{H}), 7.02(\mathrm{t}, \mathrm{J}=7.1 \mathrm{~Hz}, 1 \mathrm{H})$, $2.77-2.69(\mathrm{~m}, 2 \mathrm{H}), 2.63-2.52(\mathrm{~m}, 2 \mathrm{H}), 1.28(\mathrm{~s}, 12 \mathrm{H}), 1.24(\mathrm{~s}, 12 \mathrm{H})$.

${ }^{13} \mathrm{C}$ NMR $\left(101 \mathrm{MHz}, \mathrm{CDCl}_{3}\right) \delta 161.4,142.2,128.5,128.3,125.9,83.3,83.0,37.5,35.7,25.0,24.9$.

${ }^{11} \mathrm{~B}$ NMR $\left(128 \mathrm{MHz}, \mathrm{CDCl}_{3}\right) \delta 29.2$.

FTIR $\left(\mathrm{cm}^{-1}\right)$ 3026, 2977, 2929, 1604, 1496, 1344, 1300, 1142, 1009, 854.

HRMS (ESI) m/z: [M + H]+ Calcd for $\mathrm{C}_{22} \mathrm{H}_{35} \mathrm{~B}_{2} \mathrm{O}_{4}$ 385.2721; Found 385.2722.

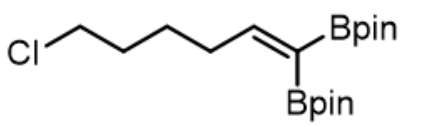

17

(17) According to General Procedure B, [(JessePhos)Pdl $]_{2}$ (82 mg, 5.0 mol \%), trifluorotoluene $(5 \mathrm{~mL}, 0.1 \mathrm{M}), N, N$-dicyclohexylmethylamine $(1.1 \mathrm{~mL}, 5.0 \mathrm{mmol})$, catBBr $(5 \mathrm{~mL}, 4.0 \mathrm{mmol})$ and alkene $(132 \mu \mathrm{L}, 1.0$ mmol) were combined under $\mathrm{N}_{2}$ and stirred at $70{ }^{\circ} \mathrm{C}$ for $24 \mathrm{~h}$. The reaction was worked up according to the General Procedure. The product was purified on boric acid impregnated silica gel $(30 \mathrm{~mL}, 1: 9$ then $3: 7$ then $1: 1$ dichloromethane : hexanes) to afford 17 as a pale yellow oil ( $272 \mathrm{mg}, 74 \%)$.

${ }^{1} \mathrm{H}$ NMR $\left(600 \mathrm{MHz}, \mathrm{CDCl}_{3}\right) \delta 6.89(\mathrm{t}, J=7.2 \mathrm{~Hz}, 1 \mathrm{H}), 3.54(\mathrm{t}, \mathrm{J}=6.7 \mathrm{~Hz}, 2 \mathrm{H}), 2.30$ (q, J = 7.3 Hz, $2 \mathrm{H}), 1.82-1.74(\mathrm{~m}, 2 \mathrm{H}), 1.58(\mathrm{dt}, \mathrm{J}=14.9,7.5 \mathrm{~Hz}, 2 \mathrm{H}), 1.29(\mathrm{~s}, 12 \mathrm{H}), 1.23(\mathrm{~s}, 12 \mathrm{H})$. 
${ }^{13} \mathrm{C}$ NMR $\left(151 \mathrm{MHz}, \mathrm{CDCl}_{3}\right) \delta 161.3,83.3,83.0,45.0,34.4,32.1,26.2,25.0,24.0$.

${ }^{11} \mathrm{~B}$ NMR $\left(193 \mathrm{MHz}, \mathrm{CDCl}_{3}\right) \delta 31.6$.

FTIR $\left(\mathrm{cm}^{-1}\right)$ 3030, 2885, 1658, 1441, 1249, 1063, 930, 839.

HRMS (ESI) m/z: [M + H]+ Calcd for $\mathrm{C}_{18} \mathrm{H}_{34} \mathrm{~B}_{2} \mathrm{O}_{4} \mathrm{Cl} 371.2332$; Found 371.2331.

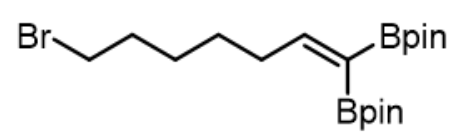

18

(18) According to General Procedure B, [(JessePhos)Pdl $]_{2}$ (82 mg, $5.0 \mathrm{~mol} \%)$, trifluorotoluene $(5 \mathrm{~mL}, 0.1 \mathrm{M}), \quad \mathrm{N}, \mathrm{N}-$ dicyclohexylmethylamine $(1.1 \mathrm{~mL}, 5.0 \mathrm{mmol})$, catBBr $(5 \mathrm{~mL}, 4.0$ $\mathrm{mmol})$ and alkene $(161 \mu \mathrm{L}, 1.0 \mathrm{mmol})$ were combined under $\mathrm{N}_{2}$ and stirred at $70{ }^{\circ} \mathrm{C}$ for $24 \mathrm{~h}$. The reaction was worked up according to the General Procedure. The product was purified on boric acid impregnated silica gel $(30 \mathrm{~mL}, 1$ : 9 then $3: 7$ then $1: 1$ dichloromethane: hexanes) to afford 18 as a colorless oil (298 $\mathrm{mg}, 69 \%$ ).

${ }^{1} \mathrm{H}$ NMR $\left(600 \mathrm{MHz}, \mathrm{CDCl}_{3}\right) \delta 6.90(\mathrm{t}, J=7.2 \mathrm{~Hz}, 1 \mathrm{H}), 3.39(\mathrm{t}, J=7.0 \mathrm{~Hz}, 2 \mathrm{H}), 2.27$ (tdt, $J=7.4$, 5.0, 2.6 Hz, 2H), $1.88-1.84(\mathrm{~m}, 2 \mathrm{H}), 1.46-1.42(\mathrm{~m}, 4 \mathrm{H}), 1.29(\mathrm{~s}, 12 \mathrm{H}), 1.23(\mathrm{~s}, 12 \mathrm{H})$.

${ }^{13} \mathrm{C}$ NMR $\left(151 \mathrm{MHz}, \mathrm{CDCl}_{3}\right) \delta 161.8,83.3,83.0,35.2,33.9,32.8,28.2,28.0,25.0,24.9$.

${ }^{11} \mathrm{~B}$ NMR $\left(193 \mathrm{MHz}, \mathrm{CDCl}_{3}\right) \delta 30.6$.

FTIR $\left(\mathrm{cm}^{-1}\right)$ 2977, 2931, 1610, 1344, 1262, 1141, 1009, 854.

HRMS (ESI) m/z: [M + H]+ Calcd for $\mathrm{C}_{19} \mathrm{H}_{36} \mathrm{~B}_{2} \mathrm{O}_{4} \mathrm{Br}$ 429.1983; Found 429.1986.

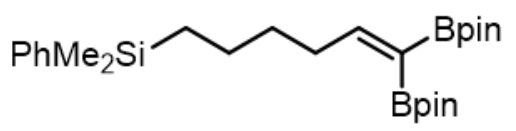

19

(19) According to General Procedure B, [(JessePhos)Pdl $\left.{ }_{2}\right]_{2}$ (82 $\mathrm{mg}, \quad 5.0 \mathrm{~mol} \%)$, trifluorotoluene $(5 \mathrm{~mL}, 0.1 \mathrm{M}), \quad \mathrm{N}, \mathrm{N}-$ dicyclohexylmethylamine $(1.1 \mathrm{~mL}, 5.0 \mathrm{mmol})$, catBBr $(5 \mathrm{~mL}, 4.0$ $\mathrm{mmol}$ ) and alkene $(218 \mathrm{mg}, 1.0 \mathrm{mmol})$ were combined under $\mathrm{N}_{2}$ and stirred at $70{ }^{\circ} \mathrm{C}$ for $24 \mathrm{~h}$. The reaction was worked up according to the General Procedure. The product was purified on boric acid impregnated silica gel $(30 \mathrm{~mL}, 1: 99$ then $5: 95$ diethyl ether : hexanes) to afford 19 as a pale yellow oil (299 $\mathrm{mg}$, $64 \%)$.

${ }^{1} \mathrm{H}$ NMR $\left(600 \mathrm{MHz}, \mathrm{CDCl}_{3}\right) \delta 7.52-7.47(\mathrm{~m}, 2 \mathrm{H}), 7.38-7.29(\mathrm{~m}, 3 \mathrm{H}), 6.91(\mathrm{t}, \mathrm{J}=7.2 \mathrm{~Hz}, 1 \mathrm{H})$, $2.25(\mathrm{q}, J=7.4 \mathrm{~Hz}, 2 \mathrm{H}), 1.46-1.42(\mathrm{~m}, 2 \mathrm{H}), 1.35-1.32(\mathrm{~m}, 2 \mathrm{H}), 1.27(\mathrm{~s}, 12 \mathrm{H}), 1.23(\mathrm{~s}, 12 \mathrm{H})$, $0.75-0.73(\mathrm{~m}, 2 \mathrm{H}), 0.24(\mathrm{~s}, 6 \mathrm{H})$.

${ }^{13} \mathrm{C}$ NMR $\left(151 \mathrm{MHz}, \mathrm{CDCl}_{3}\right) \delta 162.5,139.9,133.7,128.8,127.8,83.2,82.9,35.3,33.0,25.0,24.9$, 23.9, 15.6, -2.9.

${ }^{11} \mathrm{~B}$ NMR $\left(193 \mathrm{MHz}, \mathrm{CDCl}_{3}\right) \delta 29.6$.

${ }^{29} \mathrm{Si} \mathrm{NMR}\left(119 \mathrm{MHz}, \mathrm{CDCl}_{3}\right) \delta-3.1$.

FTIR $\left(\mathrm{cm}^{-1}\right)$ 3068, 2977, 2925, 1607, 1342, 1214, 1141, 1010, 983, 837.

HRMS (ESI) m/z: [M + H]+ Calcd for $\mathrm{C}_{26} \mathrm{H}_{45} \mathrm{~B}_{2} \mathrm{O}_{4} \mathrm{Si}$ 471.3273; Found 471.3279. 
(20) According to General Procedure B, [(JessePhos) $\left.\mathrm{Pdl}_{2}\right]_{2}$ (82 mg, 5.0<smiles>CCCCOCC=CC([B]c1ccccc1)[B]c1ccccc1</smiles>

20 $\mathrm{mol} \%)$, trifluorotoluene $(5 \mathrm{~mL}, 0.1 \mathrm{M}), \mathrm{N}, \mathrm{N}$-dicyclohexylmethylamine (1.1 $\mathrm{mL}, 5.0 \mathrm{mmol})$, catBBr $(5 \mathrm{~mL}, 4.0 \mathrm{mmol})$ and alkene $(170 \mathrm{mg}, 1.0 \mathrm{mmol})$ were combined under $\mathrm{N}_{2}$ and stirred at $70^{\circ} \mathrm{C}$ for $24 \mathrm{~h}$. The reaction was worked up according to the General Procedure. The product was purified on boric acid impregnated silica gel ( $30 \mathrm{~mL}, 1: 9$ then $3: 7$ then $1: 1$ dichloromethane : hexanes) to afford 20 as a colorless oil ( $236 \mathrm{mg}, 56 \%)$.

${ }^{1} \mathrm{H}$ NMR $\left(600 \mathrm{MHz}, \mathrm{CDCl}_{3}\right) \delta 6.92(\mathrm{t}, J=7.2 \mathrm{~Hz}, 1 \mathrm{H}), 3.42-3.33(\mathrm{~m}, 4 \mathrm{H}), 2.27$ (q, $J=7.3 \mathrm{~Hz}$, $2 \mathrm{H}), 1.58-1.52(\mathrm{~m}, 4 \mathrm{H}), 1.46-1.41(\mathrm{~m}, 2 \mathrm{H}), 1.38-1.33(\mathrm{~m}, 4 \mathrm{H}), 1.29(\mathrm{~s}, 12 \mathrm{H}), 1.23(\mathrm{~s}, 12 \mathrm{H})$, $0.91(\mathrm{t}, J=7.4 \mathrm{~Hz}, 3 \mathrm{H})$.

${ }^{13} \mathrm{C}$ NMR $\left(151 \mathrm{MHz}, \mathrm{CDCl}_{3}\right) \delta 162.4,83.2,82.9,71.0,70.8,35.5,32.0,29.8,29.1,26.1,25.0$, 24.9, 19.5, 14.1 .

${ }^{11} \mathrm{~B}$ NMR $\left(193 \mathrm{MHz}, \mathrm{CDCl}_{3}\right) \delta 31.5$.

FTIR $\left(\mathrm{cm}^{-1}\right)$ 2977, 2931, 1610, 1344, 1298, 1141, 1009, 985, 854.

HRMS (ESI) m/z: [M + H]+ Calcd for $\mathrm{C}_{23} \mathrm{H}_{45} \mathrm{~B}_{2} \mathrm{O}_{5}$ 423.3453; Found 423.3449 .

TIPSO Bpin (21) According to General Procedure B, [(JessePhos)Pdl $]_{2}$ (82 mg, $5.0 \mathrm{~mol} \%)$, trifluorotoluene $(5 \mathrm{~mL}, \quad 0.1 \mathrm{M}), \quad \mathrm{N}, \mathrm{N}-$ dicyclohexylmethylamine $(1.1 \mathrm{~mL}, 5.0 \mathrm{mmol})$, catBBr $(5 \mathrm{~mL}, 4.0$ $\mathrm{mmol}$ ) and alkene $(257 \mathrm{mg}, 1.0 \mathrm{mmol})$ were combined under $\mathrm{N}_{2}$ and stirred at $70{ }^{\circ} \mathrm{C}$ for $24 \mathrm{~h}$. The reaction was worked up according to the General Procedure. The product was purified on boric acid impregnated silica gel ( $50 \mathrm{~mL}, 1$ : 99 ethyl acetate : hexanes) to afford 21 as a yellow oil (281 $\mathrm{mg}, 55 \%)$.

${ }^{1} \mathrm{H}$ NMR $\left(600 \mathrm{MHz}, \mathrm{CDCl}_{3}\right) \delta 6.92(\mathrm{t}, J=7.2 \mathrm{~Hz}, 1 \mathrm{H}), 3.66(\mathrm{t}, J=6.4 \mathrm{~Hz}, 2 \mathrm{H}), 2.28(\mathrm{q}, J=7.3 \mathrm{~Hz}$, $2 \mathrm{H}), 1.58-1.53(\mathrm{~m}, 2 \mathrm{H}), 1.51-1.46(\mathrm{~m}, 2 \mathrm{H}), 1.28(\mathrm{~s}, 12 \mathrm{H}), 1.23(\mathrm{~s}, 12 \mathrm{H}), 1.05(\mathrm{~s}, 21 \mathrm{H})$.

${ }^{13} \mathrm{C} \mathrm{NMR}\left(151 \mathrm{MHz}, \mathrm{CDCl}_{3}\right) \delta$ 162.4, 83.2, 82.9, 63.4, 35.3, 32.9, 25.5, 25.0, 24.9, 18.2, 12.2.

${ }^{11} \mathrm{~B}$ NMR $\left(193 \mathrm{MHz}, \mathrm{CDCl}_{3}\right) \delta 30.9$.

${ }^{29} \mathrm{Si} \mathrm{NMR}\left(119 \mathrm{MHz}, \mathrm{CDCl}_{3}\right) \delta 12.0$.

FTIR $\left(\mathrm{cm}^{-1}\right)$ 2977, 2942, 2866, 1612, 1464, 1371, 1345, 1302, 1264, 1144, 1108, 1011.

HRMS (ESI) m/z: [M + H]+ Calcd for $\mathrm{C}_{27} \mathrm{H}_{55} \mathrm{~B}_{2} \mathrm{O}_{5} \mathrm{Si}$ 509.4005; Found 509.3992.

Bpin (22) A modified version of General Procedure B was used. [(JessePhos) $\left.\mathrm{Pdl}_{2}\right]_{2}$ (123 mg, $7.5 \mathrm{~mol} \%$ ), trifluorotoluene $(5 \mathrm{~mL}, 0.1$ $\begin{array}{ll}\text { Bpin } & \mathrm{M}), \mathrm{N}, \mathrm{N} \text {-dicyclohexylmethylamine }(1.1 \mathrm{~mL}, 5.0 \mathrm{mmol}), \text { catBBr }(5 \mathrm{~mL} \text {, } \\ 22 & 4.0 \mathrm{mmol}) \text { and alkene }(184 \mathrm{mg}, 1.0 \mathrm{mmol}) \text { were combined under } \mathrm{N}_{2}\end{array}$ and stirred at $70^{\circ} \mathrm{C}$ for $24 \mathrm{~h}$. The reaction was worked up according to the General Procedure. The product was purified on boric acid impregnated silica gel $(20 \mathrm{~mL}$, $2: 98$ ethyl acetate : hexanes) to afford 22 as a yellow oil (199 $\mathrm{mg}, 46 \%$ ). 
${ }^{1} \mathrm{H}$ NMR $\left(600 \mathrm{MHz}, \mathrm{CDCl}_{3}\right) \delta 6.89(\mathrm{t}, J=7.2 \mathrm{~Hz}, 1 \mathrm{H}), 4.03(\mathrm{t}, J=6.5 \mathrm{~Hz}, 2 \mathrm{H}), 2.29$ (q, J = $7.4 \mathrm{~Hz}$, $2 \mathrm{H}), 1.62(\mathrm{p}, \mathrm{J}=7.7 \mathrm{~Hz}, 2 \mathrm{H}), 1.48(\mathrm{p}, J=7.6 \mathrm{~Hz}, 2 \mathrm{H}), 1.27(\mathrm{~s}, 12 \mathrm{H}), 1.22(\mathrm{~s}, 12 \mathrm{H}), 1.17(\mathrm{~s}, 9 \mathrm{H})$.

${ }^{13} \mathrm{C}$ NMR $\left(151 \mathrm{MHz}, \mathrm{CDCl}_{3}\right) \delta 178.7,161.6,83.3,83.0,64.4,38.9,34.9,28.4,27.4,25.5,24.9$, 24.9.

${ }^{11} \mathrm{~B}$ NMR $\left(193 \mathrm{MHz}, \mathrm{CDCl}_{3}\right) \delta 31.1$.

FTIR $\left(\mathrm{cm}^{-1}\right)$ 2977, 2933, 1729, 1611, 1481. 1461, 1389, 1371, 1344, 1294, 1215, 1147, 1102 , 1033, 1010, 987, 968.

HRMS (ESI) m/z: [M + H] Calcd for $\mathrm{C}_{23} \mathrm{H}_{43} \mathrm{~B}_{2} \mathrm{O}_{6}$ 437.3246; Found 437.3237.



(23) According to General Procedure B, [(JessePhos)Pdl $]_{2}$ (82 mg, $5.0 \mathrm{~mol}$ $\%)$, trifluorotoluene $(5 \mathrm{~mL}, 0.1 \mathrm{M}), N, N$-dicyclohexylmethylamine $(1.1 \mathrm{~mL}$, $5.0 \mathrm{mmol})$, catBBr $(5 \mathrm{~mL}, 4.0 \mathrm{mmol})$ and alkene $(110 \mathrm{mg}, 1.0 \mathrm{mmol})$ were combined under $\mathrm{N}_{2}$ and stirred at $70{ }^{\circ} \mathrm{C}$ for $24 \mathrm{~h}$. The reaction was worked up according to the General Procedure. The crude product was analyzed by ${ }^{1} \mathrm{H}$ NMR by integrating against a trimethoxybenzene internal standard, resulting in a $70 \%$ assay yield. The NMR of the crude material matches the product that was prepared by our previous method. ${ }^{8}$

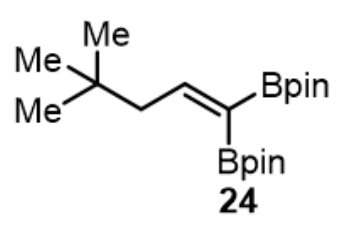

(24) According to General Procedure B, [(JessePhos)Pdl $\left.{ }_{2}\right]_{2}(82 \mathrm{mg}, 5.0 \mathrm{~mol}$ $\%)$, trifluorotoluene $(5 \mathrm{~mL}, 0.1 \mathrm{M}), N, N$-dicyclohexylmethylamine $(1.1 \mathrm{~mL}$, $5.0 \mathrm{mmol})$, catBBr $(5 \mathrm{~mL}, 4.0 \mathrm{mmol})$ and alkene $(98 \mathrm{mg}, 1.0 \mathrm{mmol})$ were combined under $\mathrm{N}_{2}$ and stirred at $70{ }^{\circ} \mathrm{C}$ for $24 \mathrm{~h}$. The reaction was worked up according to the General Procedure. The product was purified on boric acid impregnated silica gel $(30 \mathrm{~mL}, 1: 9$ then $3: 7$ then $1: 1$ dichloromethane : hexanes) to afford 24 as a white solid (232 $\mathrm{mg}, 66 \%$ ).

${ }^{1} \mathrm{H}$ NMR $\left(600 \mathrm{MHz}, \mathrm{CDCl}_{3}\right) \delta 6.97(\mathrm{t}, J=7.6 \mathrm{~Hz}, 1 \mathrm{H}), 2.17(\mathrm{~d}, J=7.6 \mathrm{~Hz}, 2 \mathrm{H}), 1.29(\mathrm{~s}, 12 \mathrm{H})$, $1.23(\mathrm{~s}, 12 \mathrm{H}), 0.92(\mathrm{~s}, 9 \mathrm{H})$.

${ }^{13} \mathrm{C}$ NMR $\left(151 \mathrm{MHz}, \mathrm{CDCl}_{3}\right) \delta$ 159.2, 83.2, 82.9, 49.7, 31.4, 29.7, 24.9, 24.8.

${ }^{11} \mathrm{~B}$ NMR $\left(193 \mathrm{MHz}, \mathrm{CDCl}_{3}\right) \delta 31.72,30.34$.

FTIR $\left(\mathrm{cm}^{-1}\right)$ 2977, 2958, 2867, 1610, 1467, 1347, 1262, 1237, 1214, 1142, 1110, 1077, 1009, 988, 966. m.p. $=56-58{ }^{\circ} \mathrm{C}$.

HRMS (ESI) m/z: [M + H] Calcd for $\mathrm{C}_{19} \mathrm{H}_{37} \mathrm{~B}_{2} \mathrm{O}_{4}$ 351.2878; Found 351.2865. 


\section{Decomposition Studies Of CatBBr:}

\section{Stability of catBBr in Toluene and Trifluorotoluene}

Experiments were run using a 1-dram vial in a nitrogen filled glovebox. 198mg of catBBr was weighed into a 1-dram vial equipped with a magnetic stir bar. $1 \mathrm{~mL}$ of solvent was added using a micropipette. The vial was capped with a septa cap and placed in an aluminum heating block and was heated at the noted temperature for 12 hours. After 12 hours, the resulting solution was transferred into a NMR tube under $\mathrm{N}_{2}$, capped and sealed with parafilm. ${ }^{11} \mathrm{~B}$ NMR was obtained using $\mathrm{CDCl}_{3}$ as the solvent. The catBBr peak was observed around $27.5 \mathrm{ppm}, \mathrm{B}_{2} \mathrm{Cat}_{3}$ was observed around $22.5 \mathrm{ppm}$, and an unidentifiable decomposition product was observed at 20.9 ppm.



Figure S1. Decomposition Studies of catBBr. 


\section{General Procedure for the Time Study Experiment:}

\section{Experimental Procedure:}

All experiments are completed in a nitrogen-filled glovebox. A 2-dram vial was charged with [(JessePhos) $\left.\mathrm{Pdl}_{2}\right]_{2}(10.3 \mathrm{mg}, 2.5 \mathrm{~mol} \%$ ) and trifluorotoluene $(1.25 \mathrm{~mL})$. The resultant solution was stirred at room temperature for 10 minutes. Thereafter, $N, N$-dicyclohexylmethylamine (226 $\mu \mathrm{L}, 5.0$ equiv), 4-tert-butylstyrene (.25 mmol, 1.0 equiv), catBBr (198 mg, 4.0 equiv), and toluene $(1.25 \mathrm{~mL})$ were added sequentially. The vial was capped, sealed with tape, and placed into a preheated aluminum heating block at $70{ }^{\circ} \mathrm{C}$, where it was allowed to stir for $4 \mathrm{~h}$. Every 30 minutes, a $20 \mu \mathrm{L}$ aliquot of the reaction mixture was taken and quenched with pinacol. The crude mixture was dissolved in $500 \mu \mathrm{L}$ of $\mathrm{CDCl}_{3}$, was transferred to an NMR tube and was used for ${ }^{1} \mathrm{H}$ NMR analysis. Starting material and product peaks were integrated, and the resulting concentrations reported are a direct comparison of the integral ratio of reactant and product materials present in the crude NMR mixture.

\section{Studies Towards the Borylation of Trans-Monoboryl Alkenes:}

\section{Experimental Procedure:}

In a nitrogen filled glovebox, a 2-dram vial was charged with [(JessePhos) $\left.\mathrm{Pdl}_{2}\right]_{2}$ (10.3 mg, $2.5 \mathrm{~mol}$ $\%)$ and trifluorotoluene $(1.25 \mathrm{~mL})$. The resultant solution was stirred at room temperature for 10 minutes. $N, N$-dicyclohexylmethylamine ( $226 \mu \mathrm{L}, 5.0$ equiv), boryl-styrene $(0.25 \mathrm{mmol}, 1.0$ equiv), catBBr (99 mg, 2.0 equiv), and toluene $(1.25 \mathrm{~mL}$ ) were added sequentially. The vial was capped, sealed with tape, and placed into a pre-heated aluminum heating block at $70{ }^{\circ} \mathrm{C}$, where it was allowed to stir for $4 \mathrm{~h}$. At that time, the vial was removed from the heating block and allowed to cool to room temperature. Trimethoxybenzene was added as an internal standard, then the solvent was removed under reduced pressure. The crude mixture was then analyzed by NMR. ${ }^{1} \mathrm{H}$ NMR indicted that presence of a single major product in $\sim 95 \%$ yield verses the internal standard and is consistent with structure $\mathbf{S 1}$ shown below. Unfortunately, attempts to isolate the product for definitive characterization failed due to instability on silica gel, presumably due to the sensitive nature of the Bcat group. NMR spectra of the crude mixture are provided below.

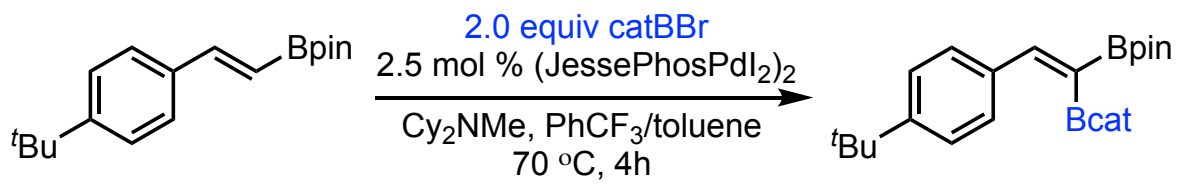

S1

(tentative structure, 95\%) 


\section{References:}

(1) Pangborn, A. B.; Giardello, M. A.; Grubbs, R. H.; Rosen, R. K.; Timmers, F. J. Organometallics 1996, 15, 1518.

(2) Reid, W. B.; Watson, D. A. Org. Lett. 2018, 20, 6832.

(3) McAtee, J. R.; Yap, G. P. A.; Watson, D. A. J. Am. Chem. Soc. 2014, 136, 1016.

(4) Krause, S. B.; McAtee, J. R.; Yap, G. P. A.; Watson, D. A. Org. Lett. 2017, 19, 564.

(5) Martin, S. E. S.; Watson, D. A. J. Am. Chem. Soc. 2013, 135, 13330.

(6) Dong, X-Y.; Cheng, J-T.; Zhang, Y-F.; Li, Z-L.; Zhan, T-Y.; Chen, J-J.; Wang, F-L.; Yang, N-Y.; Ye, L.; Gu, Q-S.; Liu, X-Y. J. Am. Chem. Soc. 2020, 142, 9501-9509.

(7) Waser, J.; Gaspar, B.; Nambu, H.; Carreira, E. M. J. Am. Chem. Soc. 2006, 128, 11693.

(8) Reid, W. B.; Spillane, J. J.; Krause, S. B.; Watson, D. A. J. Am. Chem. Soc. 2016, 138, 5539.

(9) Wrackmeyer, B. Prog. Nucl. Magn. Reson. Spectrosc. 1979, 12, 227.

(10) Hitosugi, S.; Tanimoto, D.; Nakanishi, W.; Isobe, H. Chem. Lett. 2012, 41, 972.

(11) Gallagher, W. P.; Vo, A. Org. Process Res. Dev. 2015, 19, 1369.

(12) As noted in the general experimental details, in some cases we repeated reactions multiple times to insure reproducibility. In these cases, we have reported the average yield in the main body of the paper, and a representative experimental run in the Supporting Information.

\section{Spectral Data:}



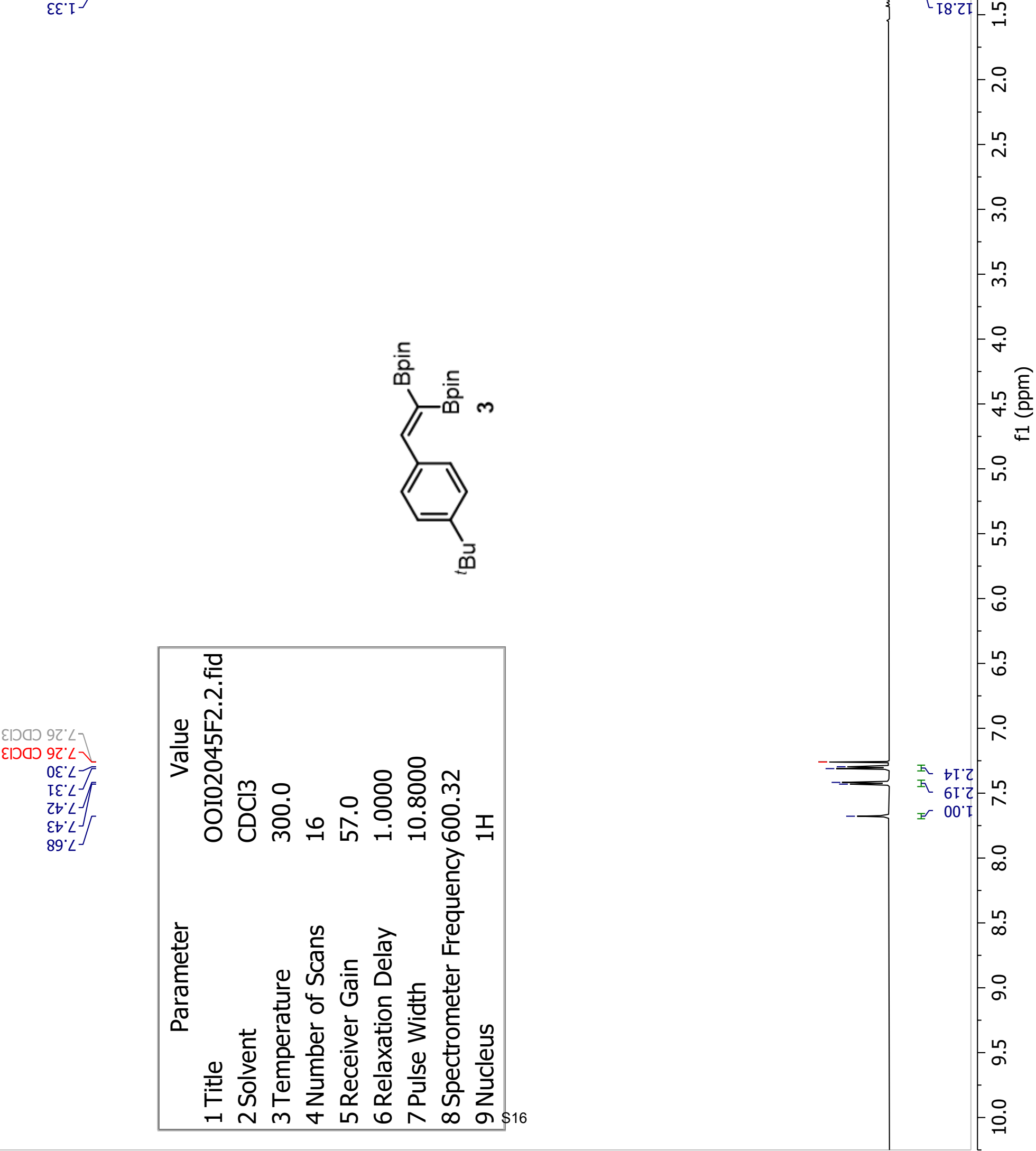
$\downarrow 8^{\circ} \downarrow \tau$
$\varepsilon 0^{\circ} s \zeta$

乙ち. TE-

$6 L^{\circ} \dagger \varepsilon-$

عادم $\varsigma 6^{\circ} 9 \angle>$

हागव 9ा" $\angle L]$

हागح 9I' $L L \frac{1}{]}$

५ ' $\varepsilon 8$

$\angle 9^{\circ} \varepsilon 8$
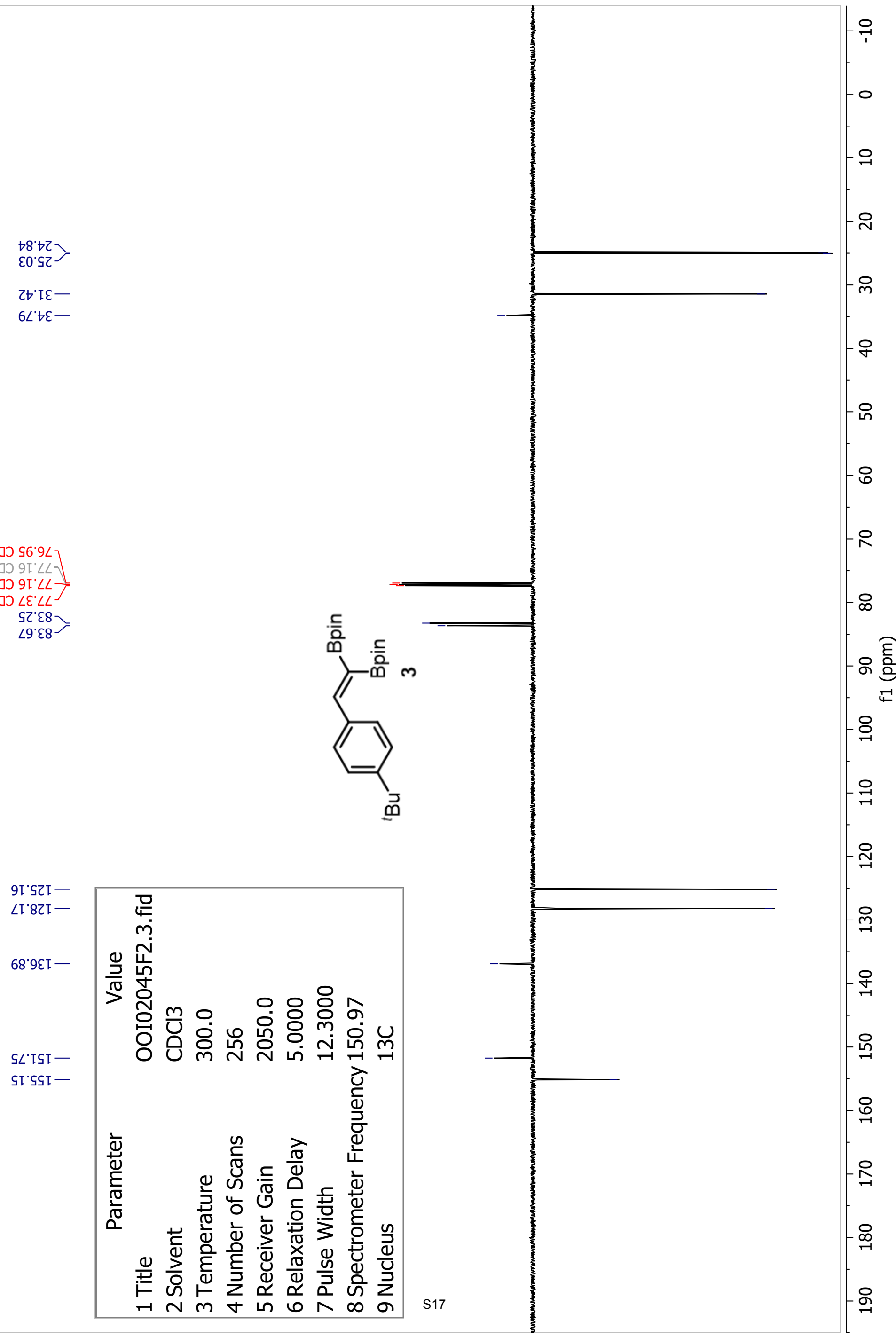

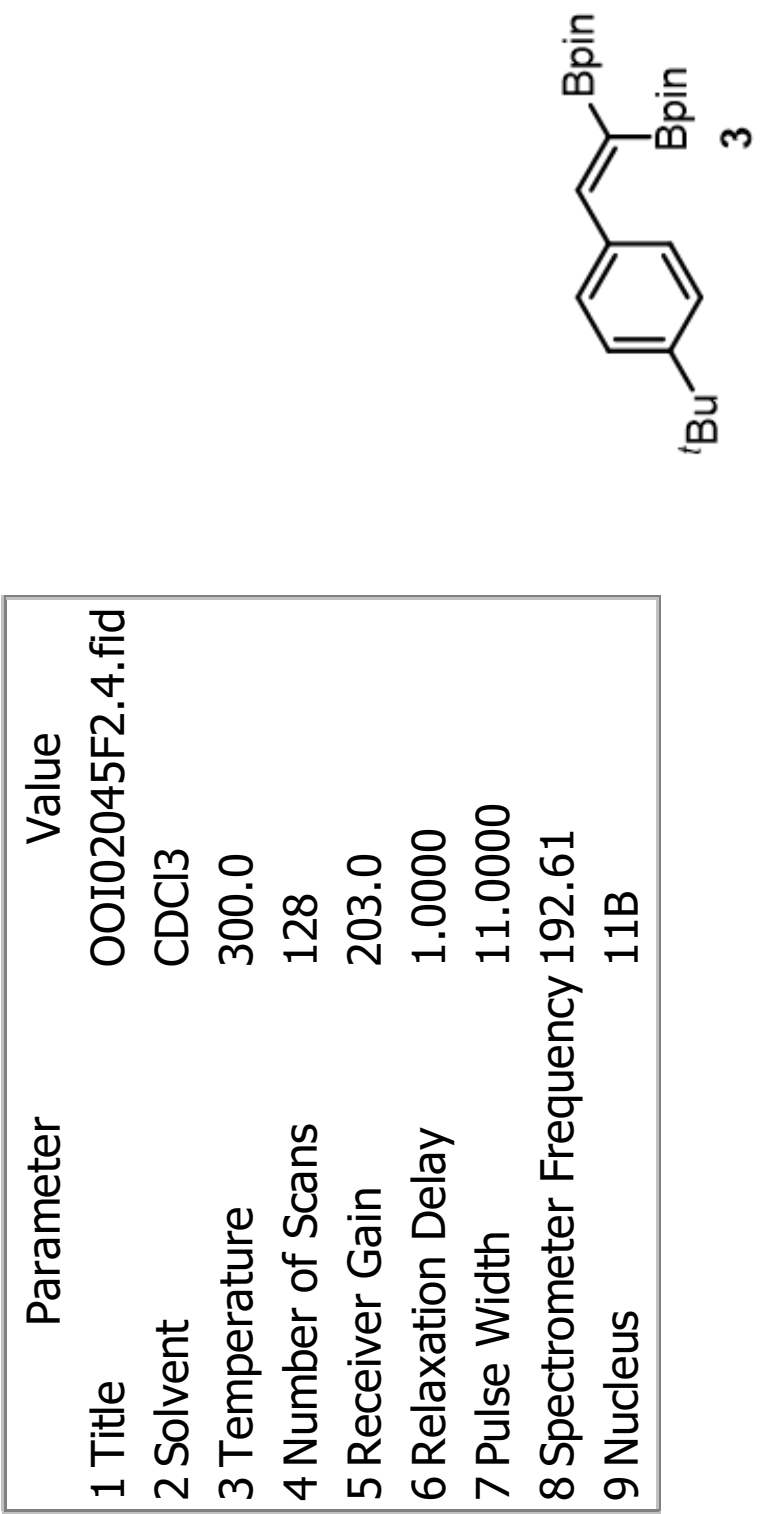

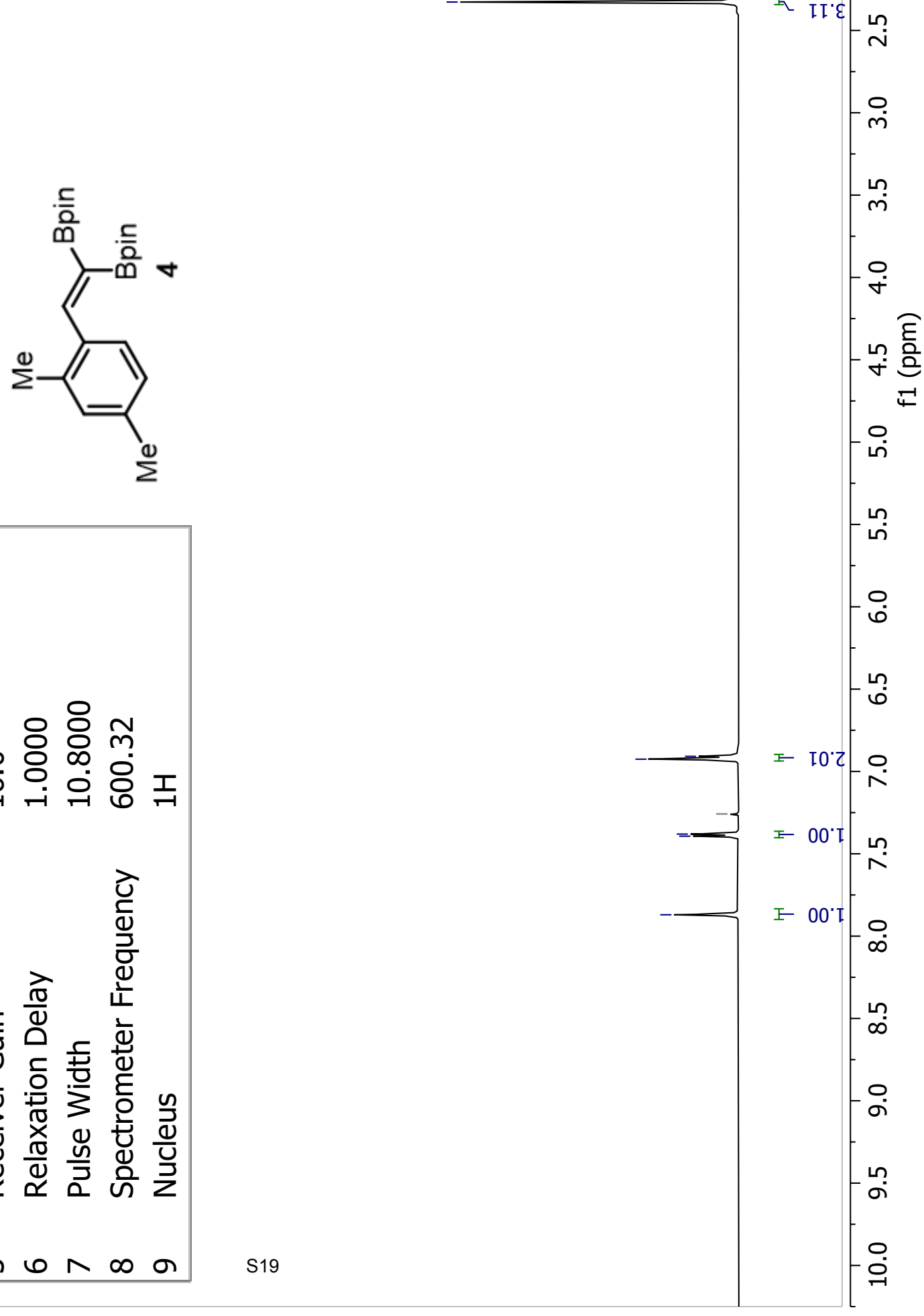


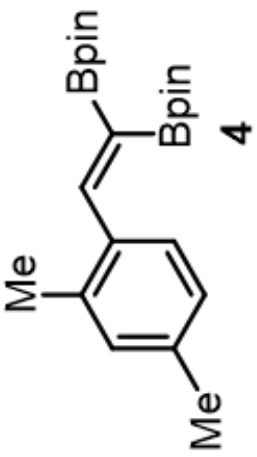

$\varepsilon I^{\prime} \varepsilon 8$
ح' ${ }^{\prime} \varepsilon 8^{-}$

0t.9ZI $\varepsilon L ' \angle Z I-$ 乙8.0हI॰ ${ }^{\prime} 9 \varepsilon[$ โ $9 \varepsilon$ I

IZ'8EI
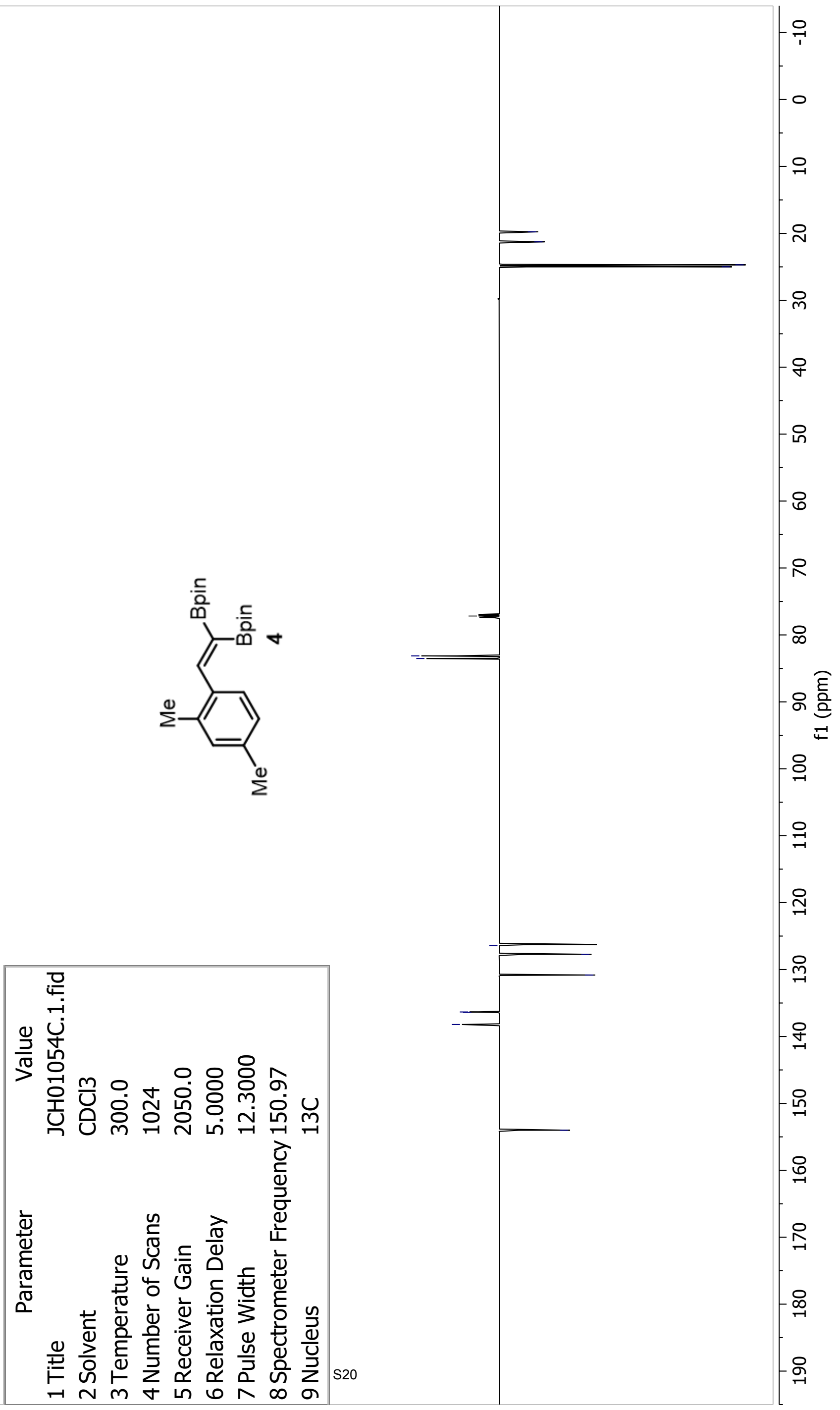


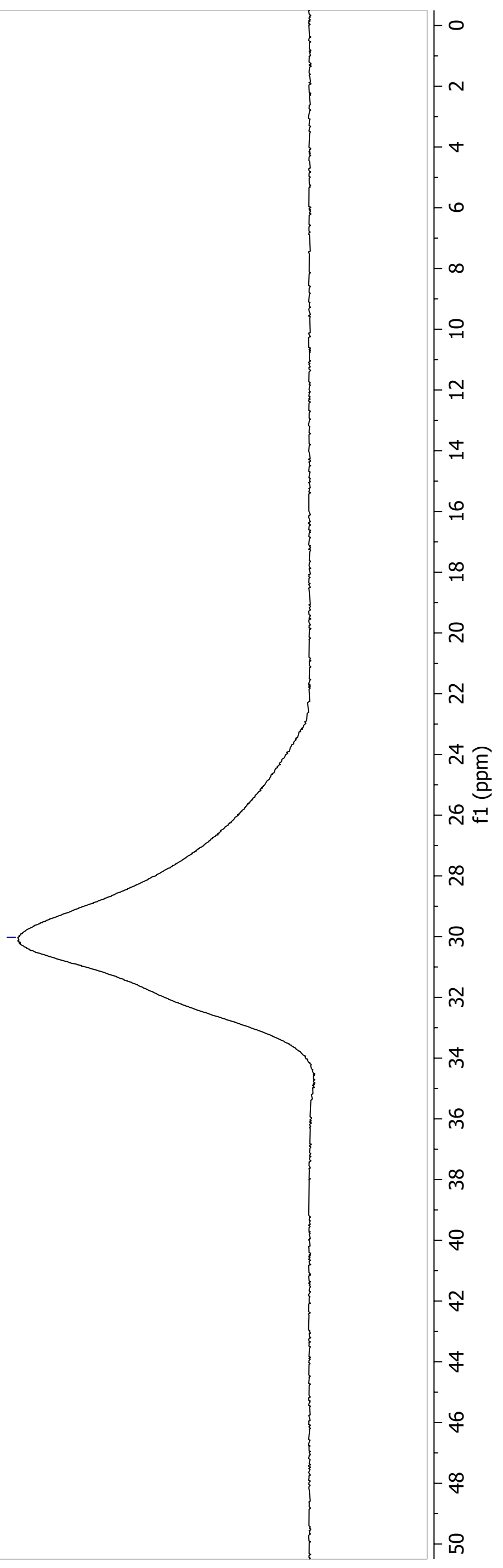




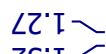

乙E' $\mathrm{I}$

$08^{\circ} \varepsilon-$

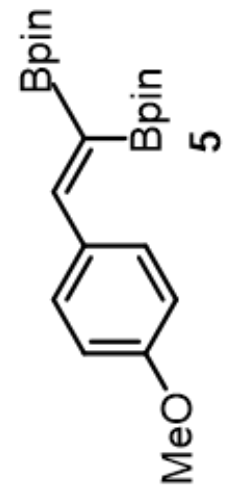

हाวดว 92: $\angle$ ᄀ

\section{>}

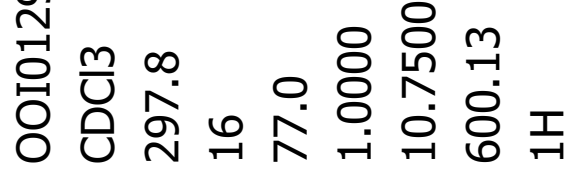

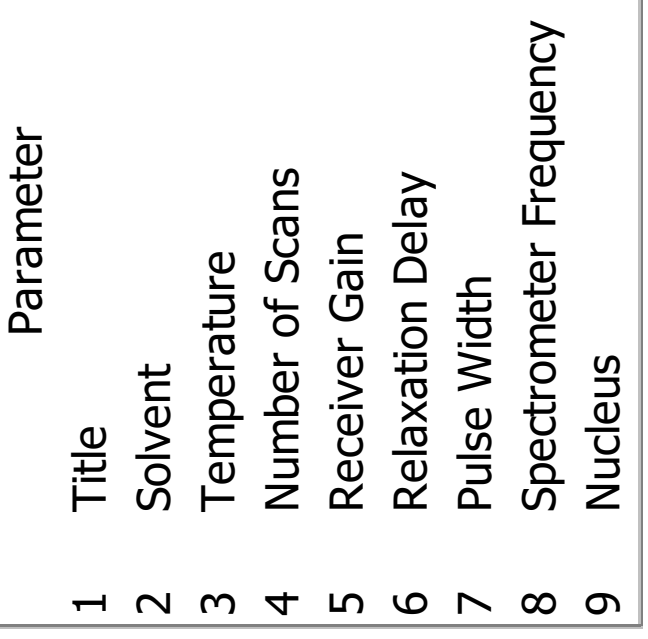


$\downarrow 8^{\circ} \downarrow 乙$
$20 . \varsigma 2$

Ot'ss-
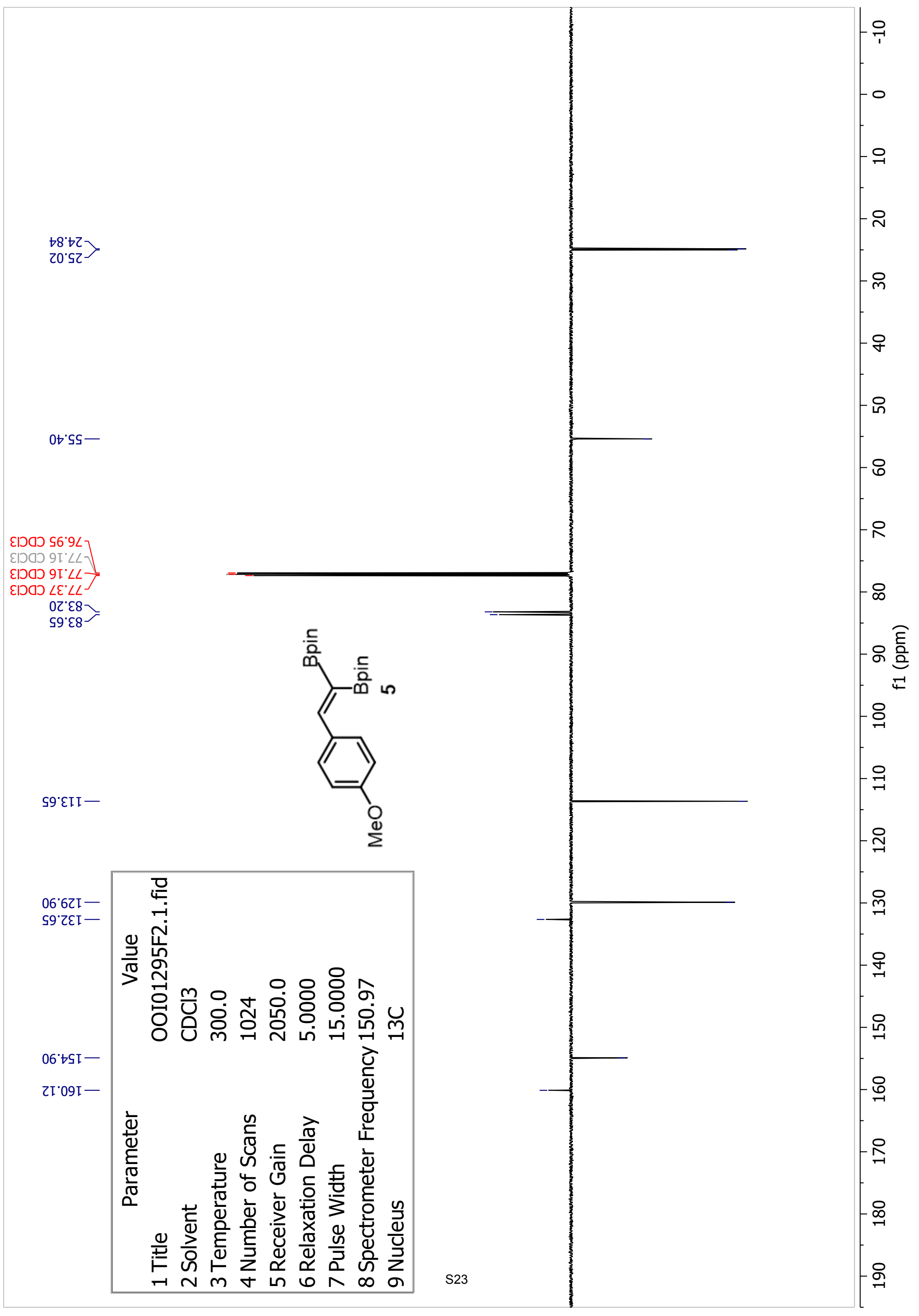



$\frac{\frac{1}{d}}{\frac{d}{d}} \frac{\frac{1}{0}}{\frac{0}{\sigma}}$
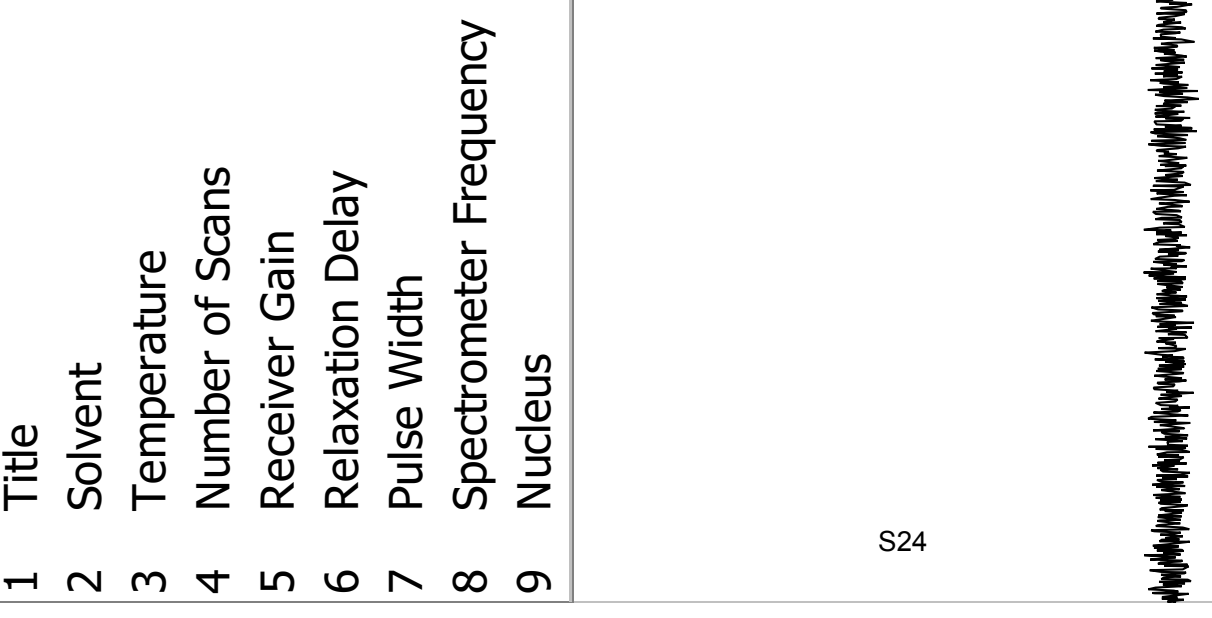


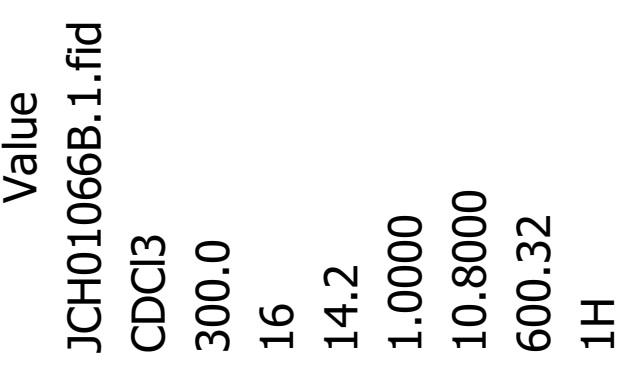

$29^{\circ} \mathrm{L}$



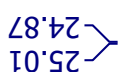

등

S6'28

รด'ย8

S9.'II -

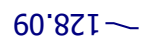

ع6.6ZI-

89.

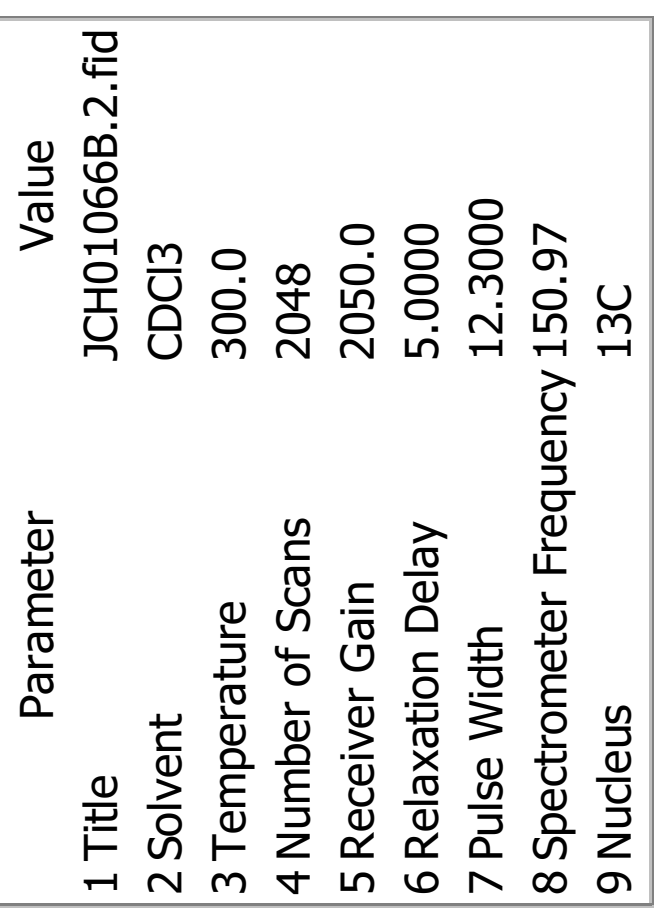

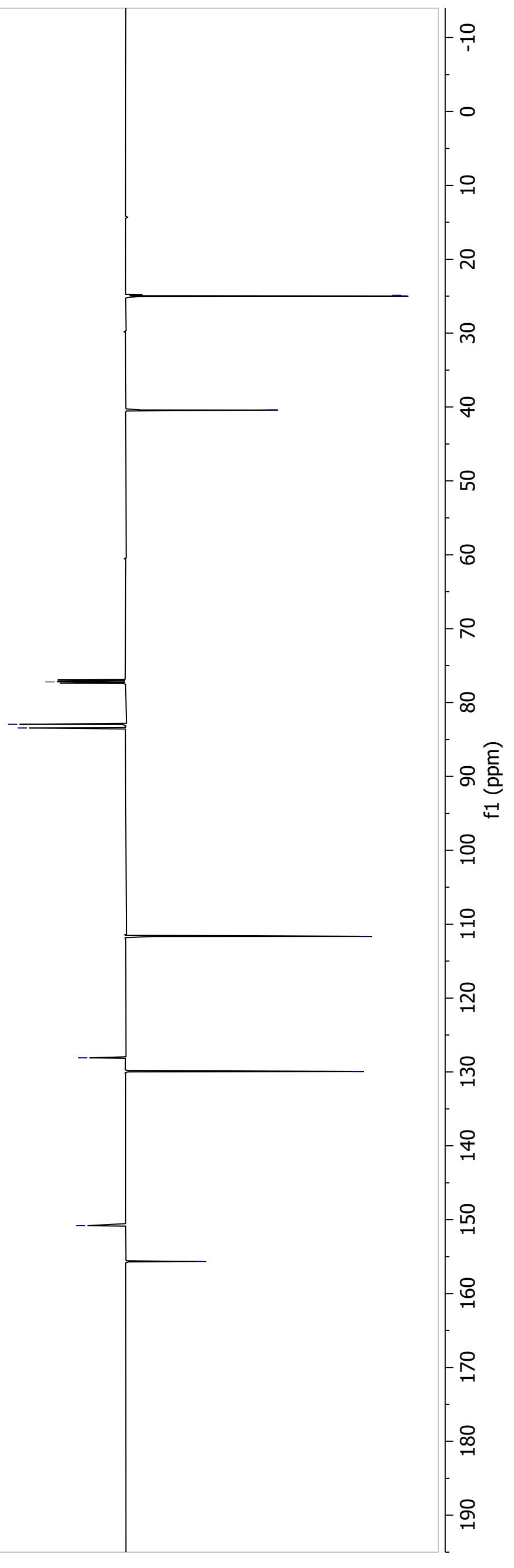




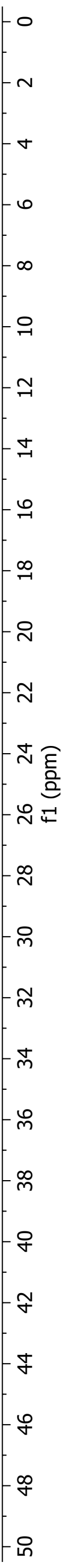






โย 'โ
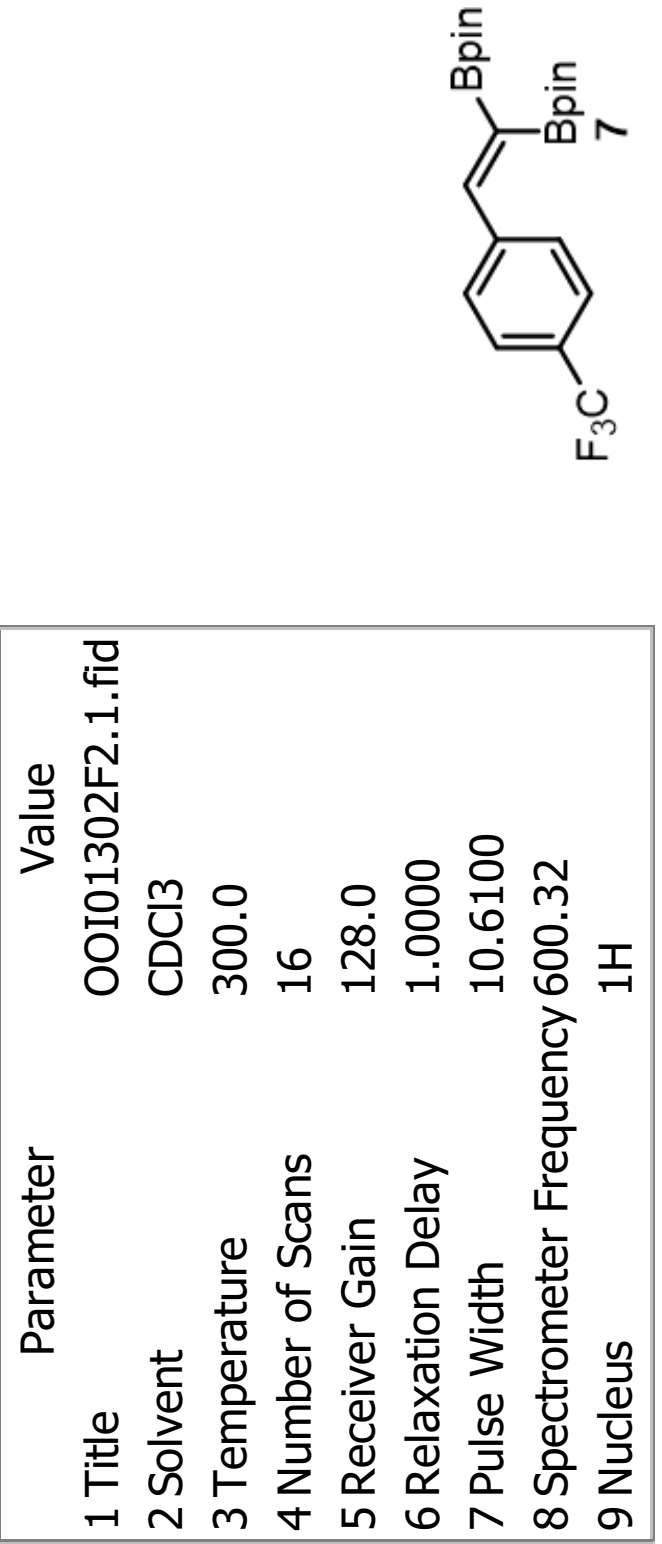




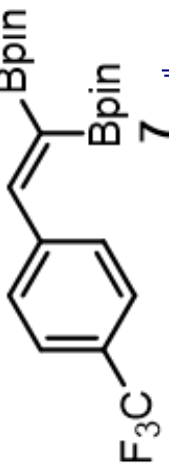



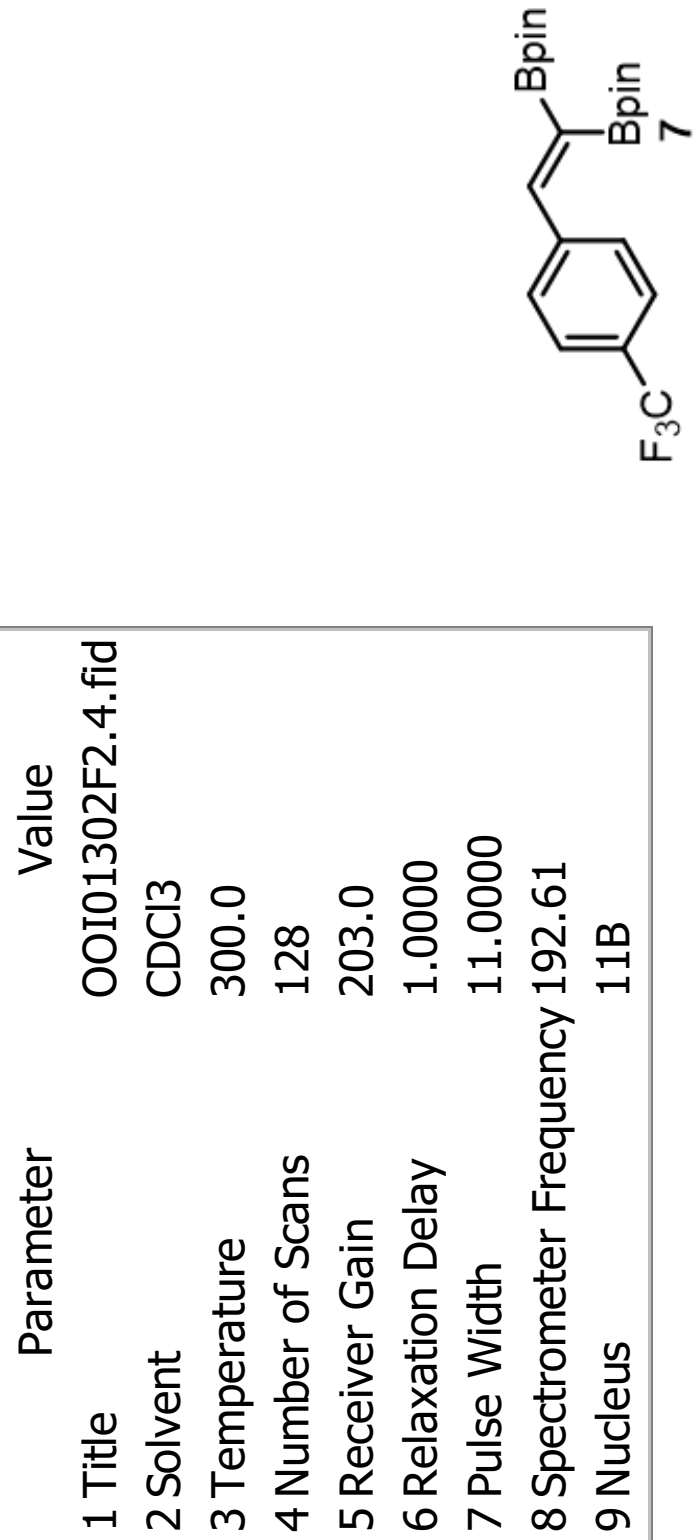


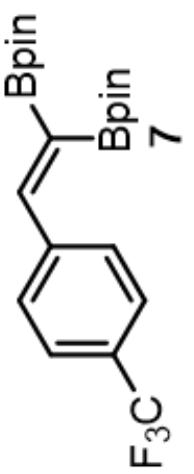

95'29--

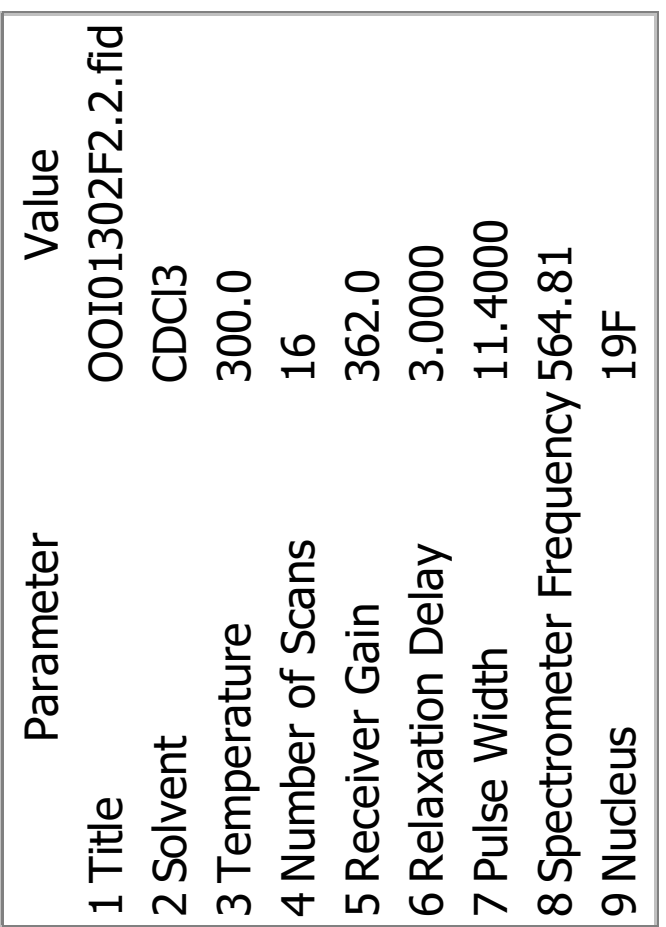


sE'

$9 \varepsilon \cdot b]$

$\angle \varepsilon^{\prime} \circ-$

$8 \varepsilon^{\prime} \circ$

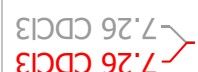
$\varepsilon S^{\circ} L$ $\downarrow S^{\prime} L$ I $\angle L^{\prime} L$ $\angle 6^{\circ} \angle>$ $86^{\circ} L^{\prime}$

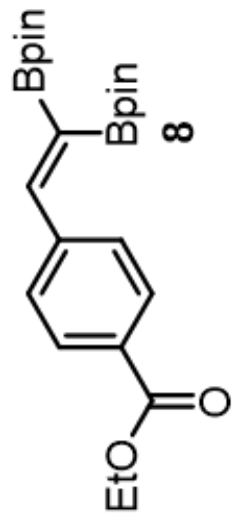


$8 b^{\circ}+5$

$28 \cdot \bullet \tau$
20.52

80.19-

عાગم ૬ $6^{\circ} 9 \angle$

हागव 9.' $\angle L]$



$95^{\circ} \varepsilon 8$

86. $\varepsilon 8$
\[ I^{\circ} 8 \mathrm{LI} \]
I9.6ZI

I9.6ZI
IZ'0EI

$86^{\circ} \varepsilon \triangleright I-$

LL'ESI -

SS'99I -





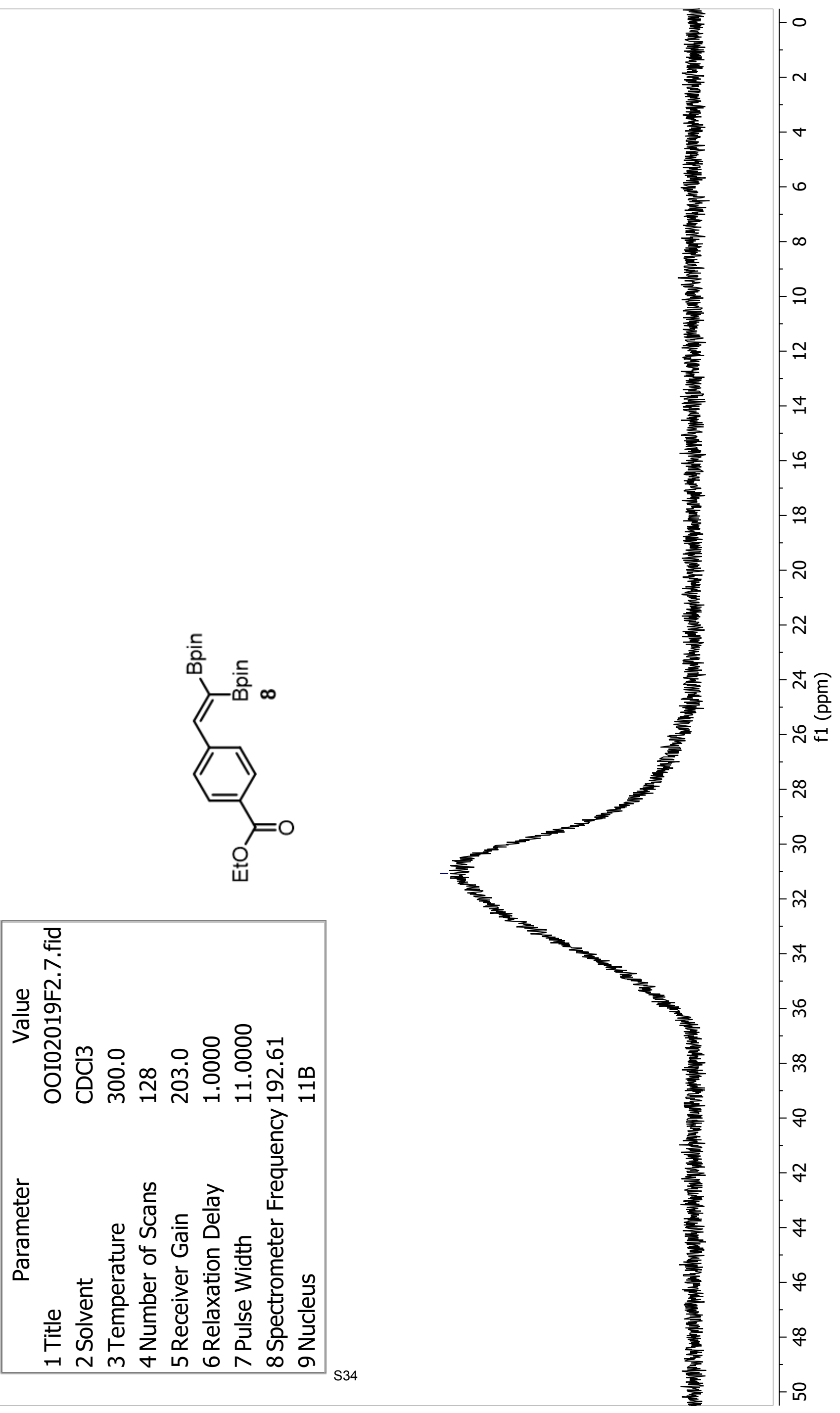
$\angle Z^{\prime} I>$

IE

$\left.\begin{array}{l}96^{\circ} 9 \\ 96^{\circ} 9 \\ \angle 6^{\circ} 9 \\ \angle 6^{\circ} 9 \\ \angle 6^{\circ} 9 \\ 86^{\circ} 9 \\ 86^{\circ} 9 \\ 86^{\circ} 9 \\ 66^{\circ} 9 \\ 66^{\circ} 9 \\ 00^{\circ} \angle\end{array}\right]$

हागم $00^{\circ} \angle$

हागऽ $\left.92^{\circ} /\right]$



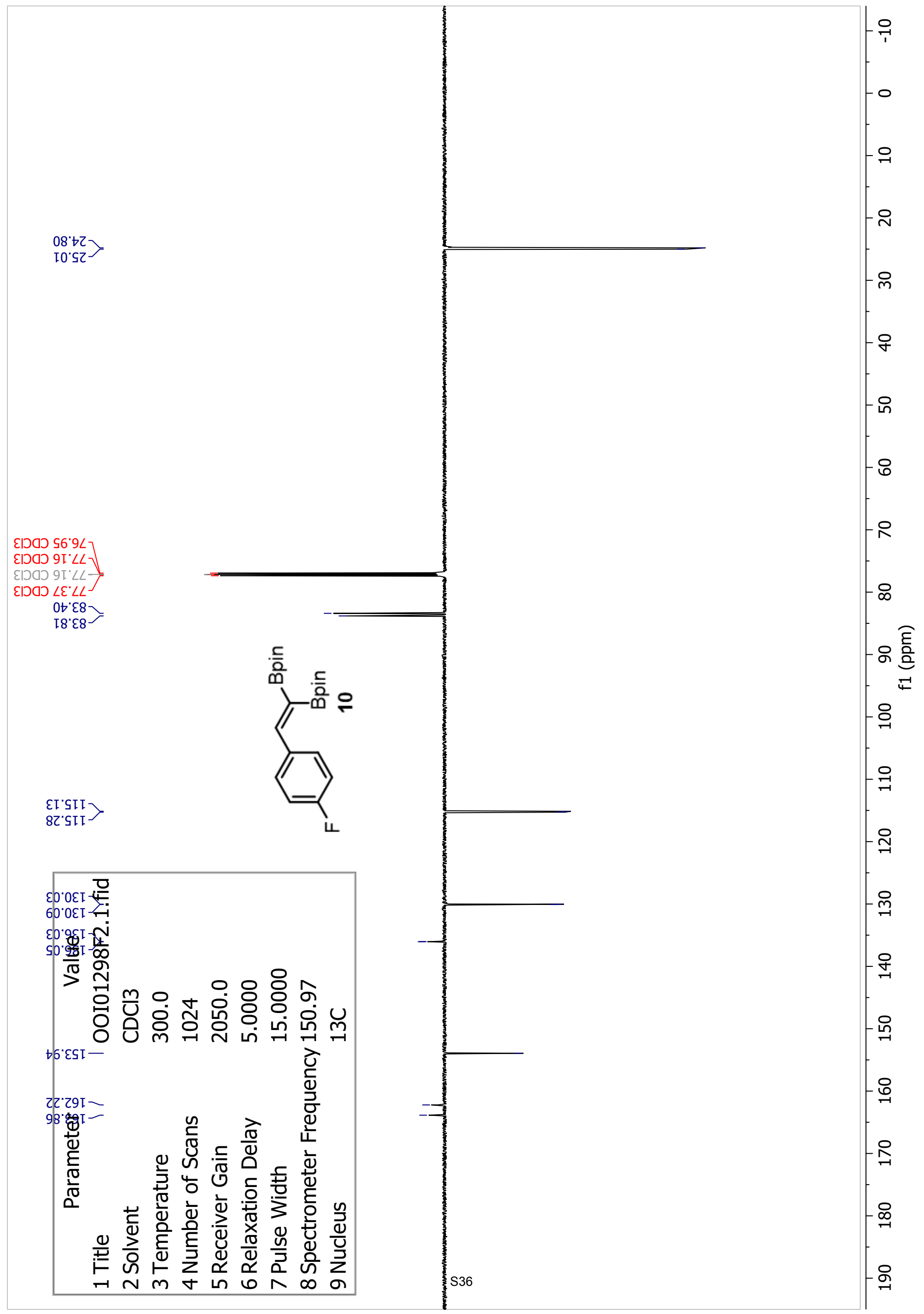



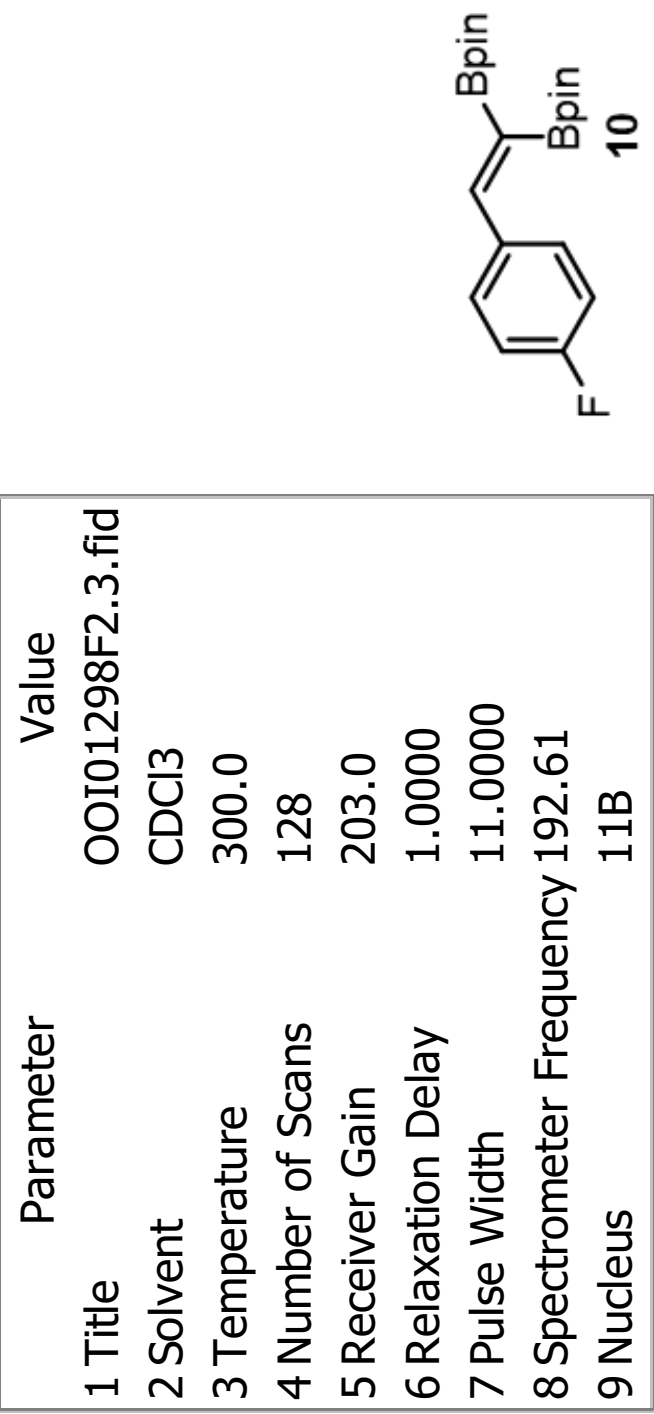





$82^{\prime} \mathrm{I}$

โE '
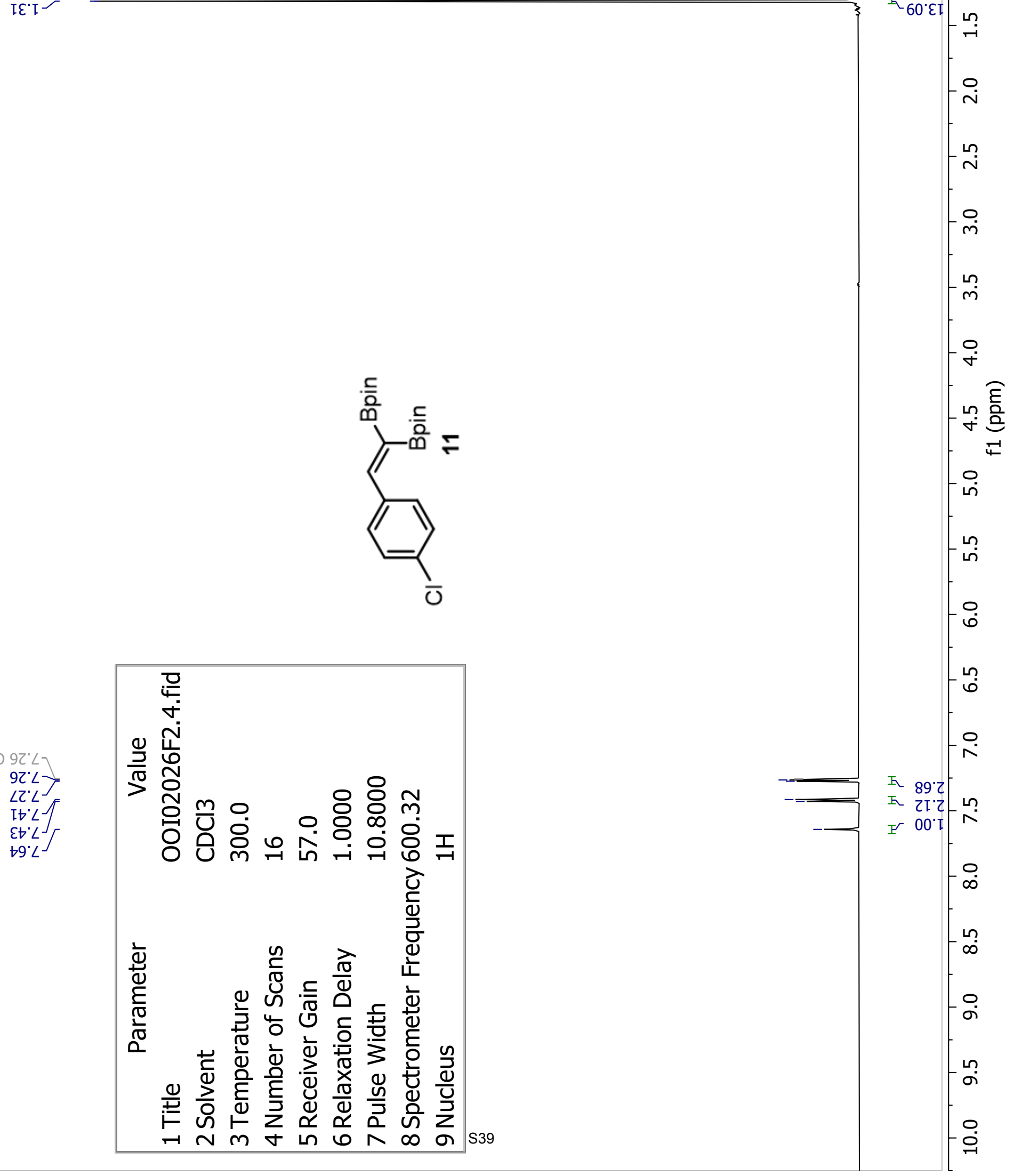
$08^{\circ} \downarrow 乙$
$00^{\circ} \varsigma$

हागه 56.9L

हागح 9ा' $\angle L]$

$\left.\begin{array}{l}\text { हाग } 9 T^{*} \angle L \\ \text { हाग } \angle \varepsilon^{\circ} \angle L\end{array}\right]$

$\angle t^{\circ} \varepsilon 8$

$88^{\circ} \varepsilon 8^{\prime}$
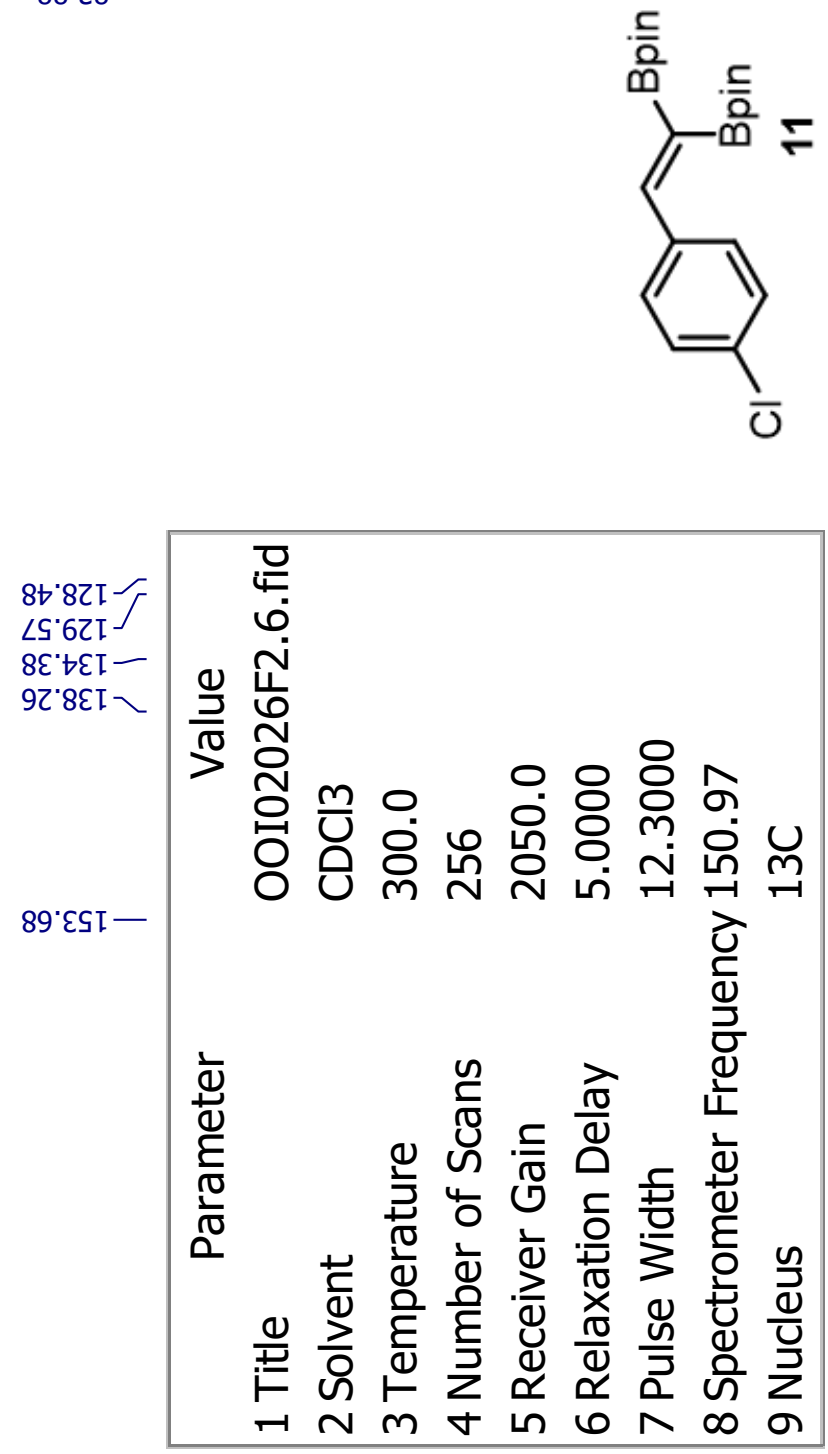

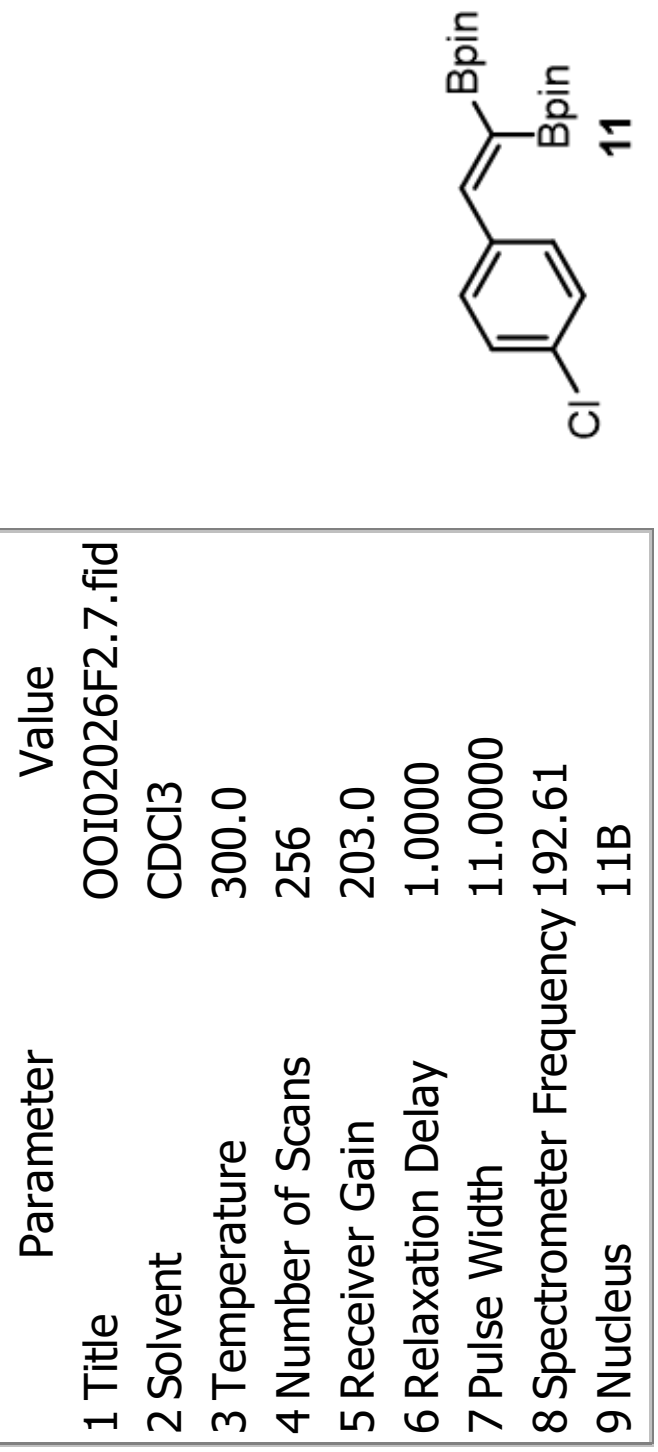
$\angle Z^{\prime} I=$
$I E^{\prime} I$





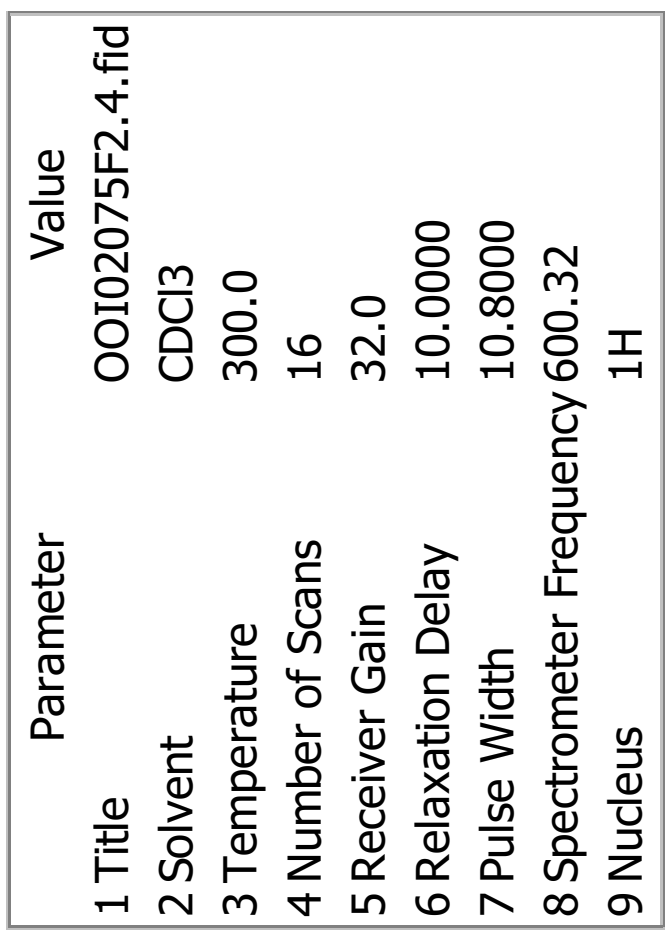




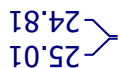

हागه 56.9L

हागه 9I' $\angle L]$

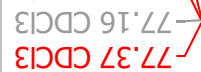

$86^{\circ} \varepsilon 8$

$68^{\circ} \varepsilon 8^{\prime}$



89.2ZI-

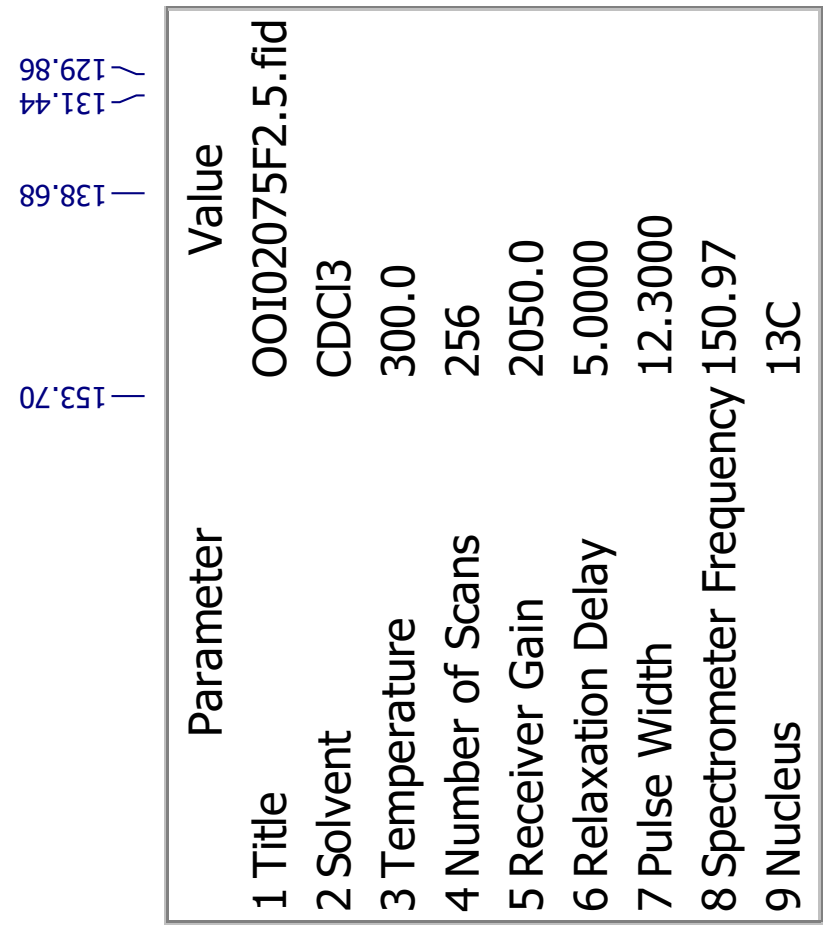




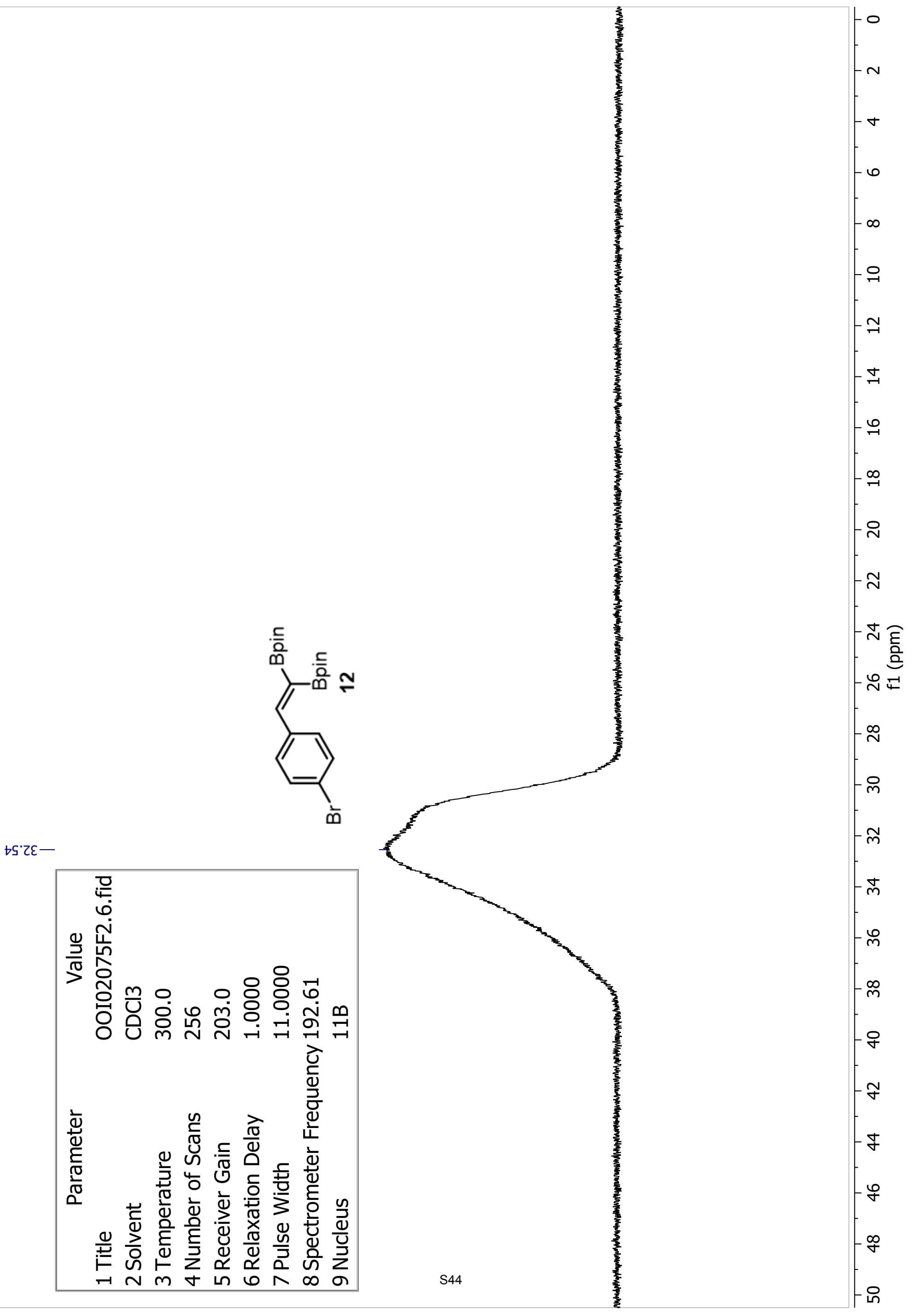


$\angle Z^{\prime} I \simeq$

$\varepsilon \varepsilon^{\prime} \mathrm{T}-$

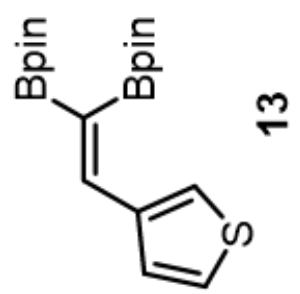

IZ'L]

$\left.2 Z^{\circ} \angle\right]$

$\left.2 Z^{\circ} \angle\right]$

हागه $\left.92^{\circ} \angle\right]$

हाग๐ 92" $\angle$ ]

IE $\angle$

乙ع: $\measuredangle]$

乙E' $\angle]$

$t t^{\circ} L$

$t t^{\circ} L$

$t t^{\circ} L$

$S t^{\circ} L$
$59^{\circ} L$

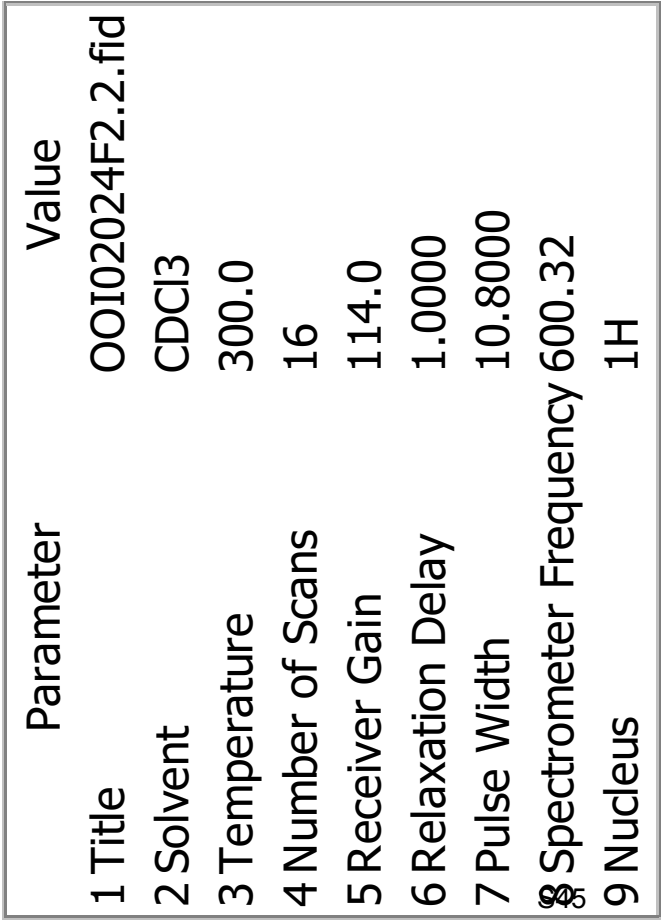


$\sqcup 6^{\circ} \bullet 乙$
10.52

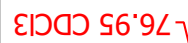

हाग๐ 9[" $\angle L]$

हागح 9I' $\angle L \frac{1}{J}$

$6 Z^{\prime} \varepsilon 8$

$9 L^{\prime} \varepsilon 8$

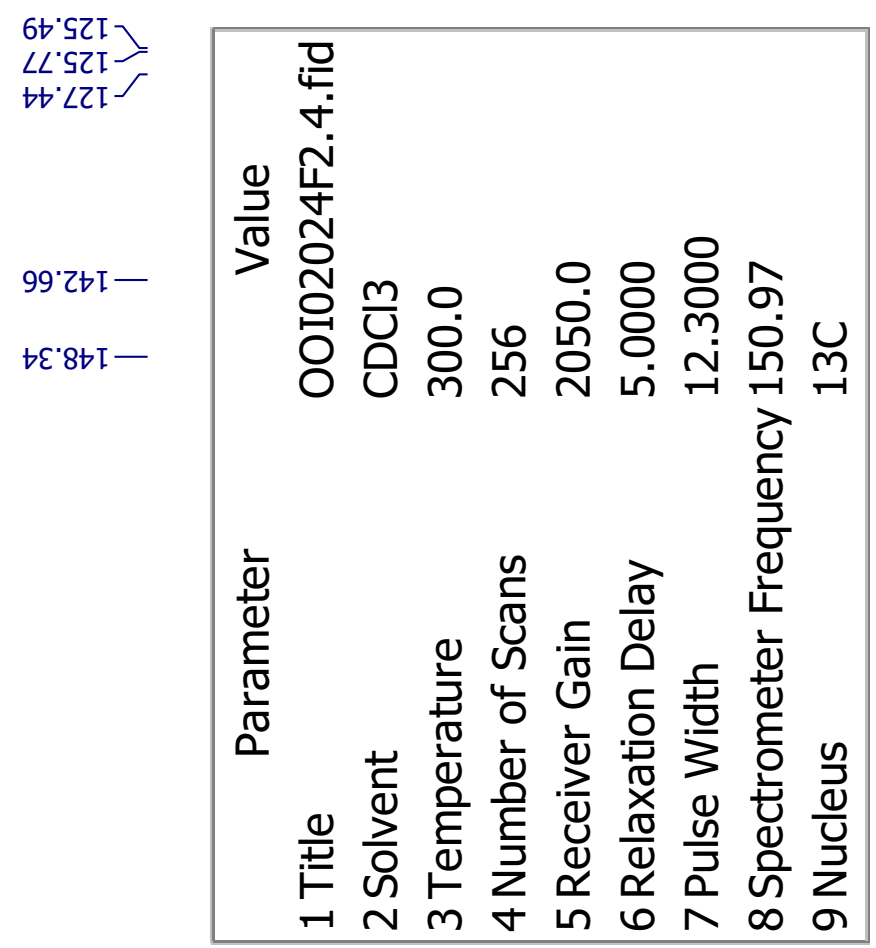

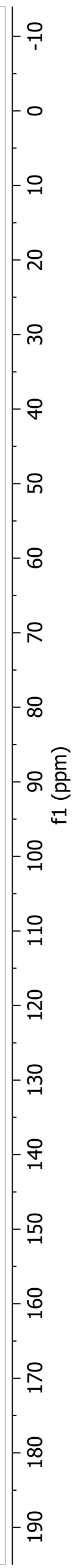



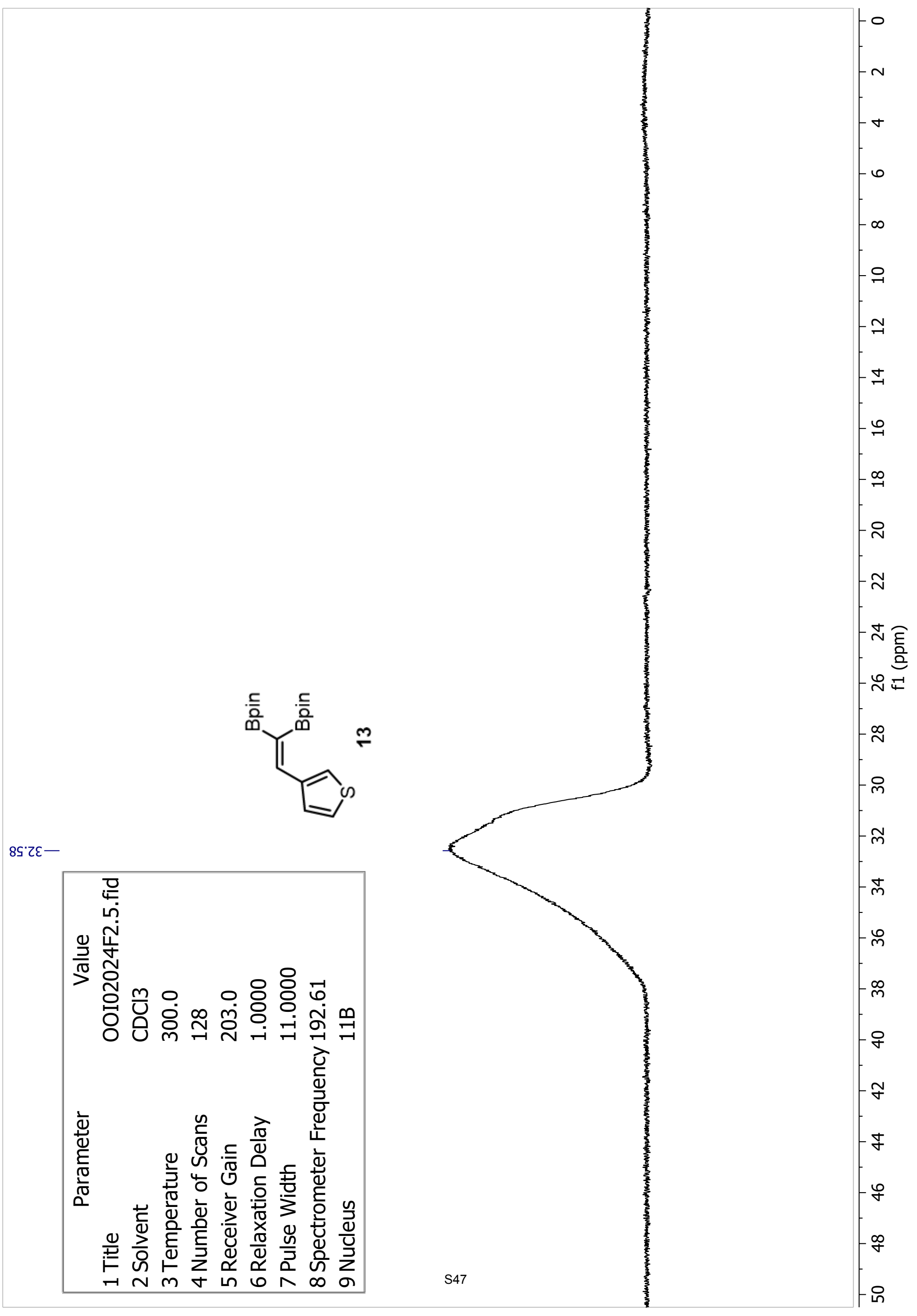
$82^{\circ} \mathrm{T}-$

$\angle \mathcal{E}^{\prime} T-$

ฉะ'z-

$8{ }^{2} \cdot \mathrm{L}$

$0 Z^{\circ} \angle$
$\mathrm{ZZ} L$

$Z Z^{\circ} \angle$

$\varepsilon Z^{\circ} L$

$\Delta Z^{\circ} \angle$

$\Delta Z^{\prime} \angle$

हागه $9 Z^{\circ} \angle$

हागऽ $9 z^{\circ} \angle$

$\left.87^{\circ} \angle\right]$

$\left.6 Z^{\prime} \angle\right]$

$\left.62^{\circ} \angle\right]$

$0 \varepsilon:$

โE $\angle$ J

$\left.\angle 9^{\circ} \angle\right]$

$89^{\circ} \mathrm{L}$

$Z \angle L$

$\left.\varepsilon L^{\circ} L\right][$

$t \angle L^{\circ} L$

$26^{\circ} \mathrm{L}$

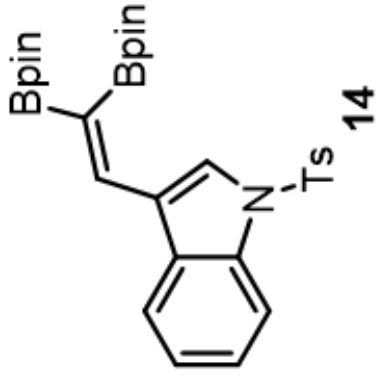

눈

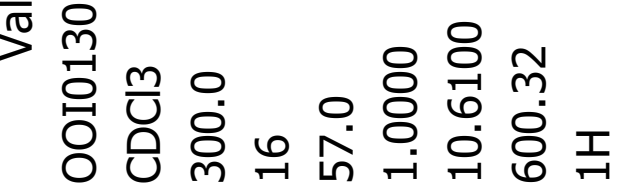

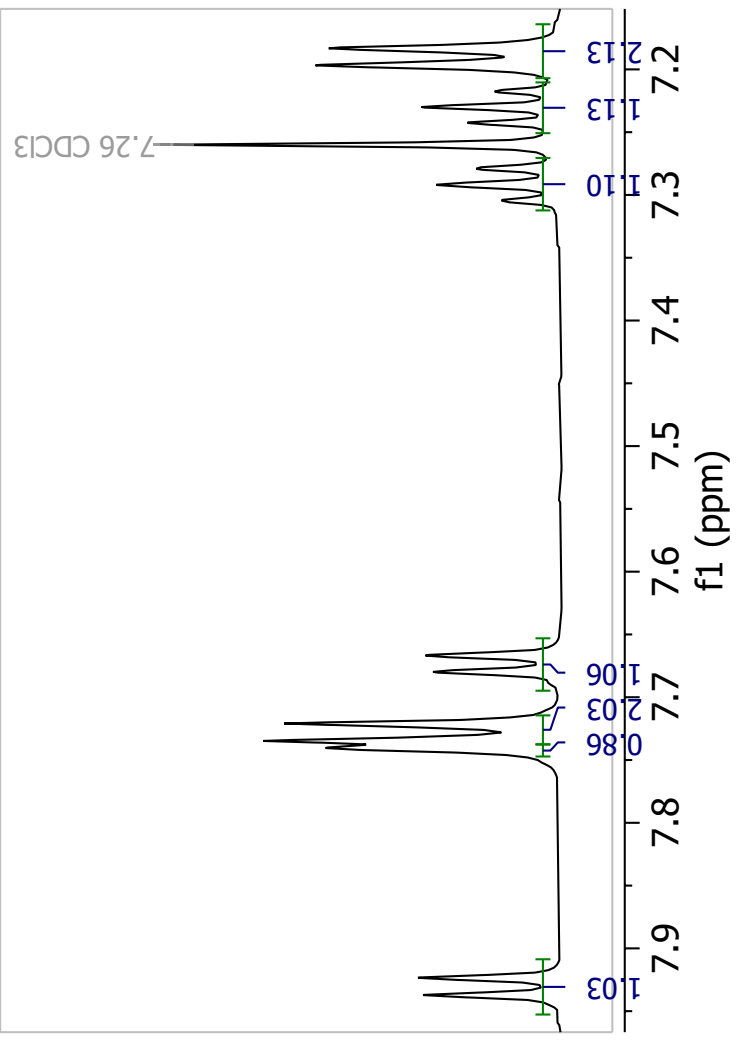

II 8

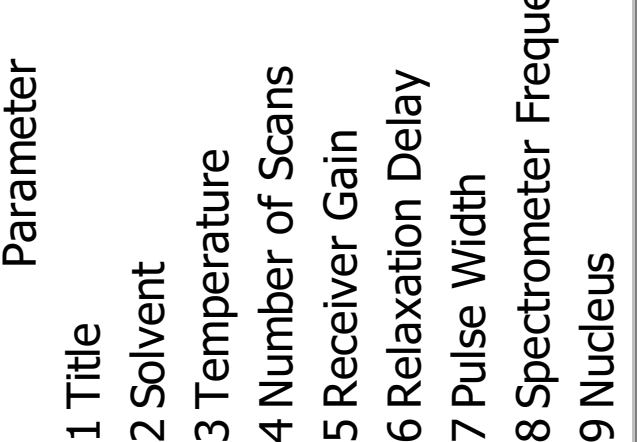


$\angle 9^{\circ} \mathrm{IZ}$

I0'SZ

EO'sZ

ع|วه ऽ $6^{\circ} 9<$

हागCว 9I' $\angle L]$

हाગه 9I: $\angle L \frac{1}{J}$

$8 \varepsilon^{\prime} \varepsilon 8$

โ0 $\vdash 8$

09'ยII -

66.6II]

Z8'ZZI

$\angle b^{\prime} \varepsilon Z I-$

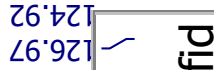

๑8.6Z1

ธ6เฉI ए

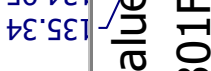

$99^{\circ} \varepsilon+1 \geqslant$

60 st

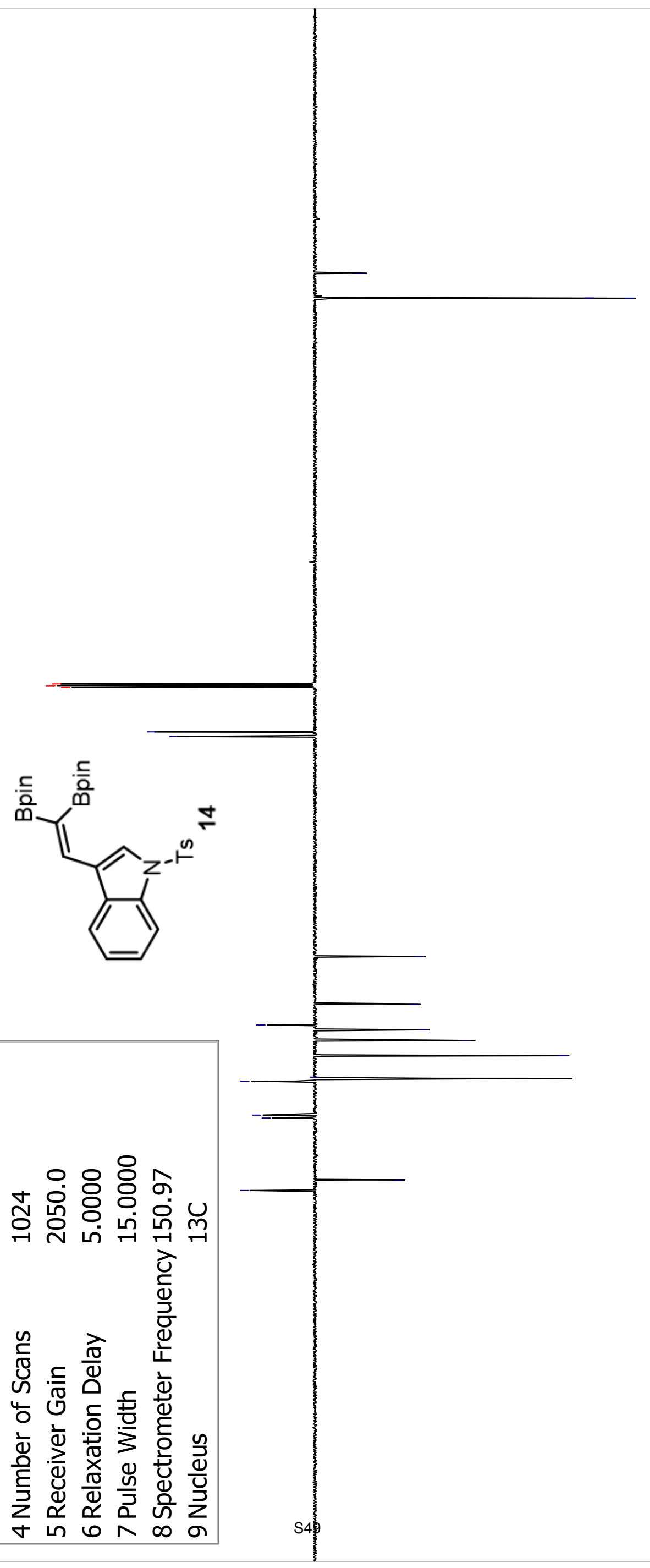

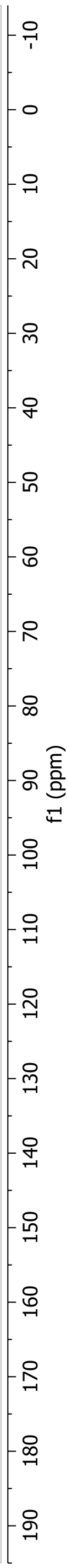



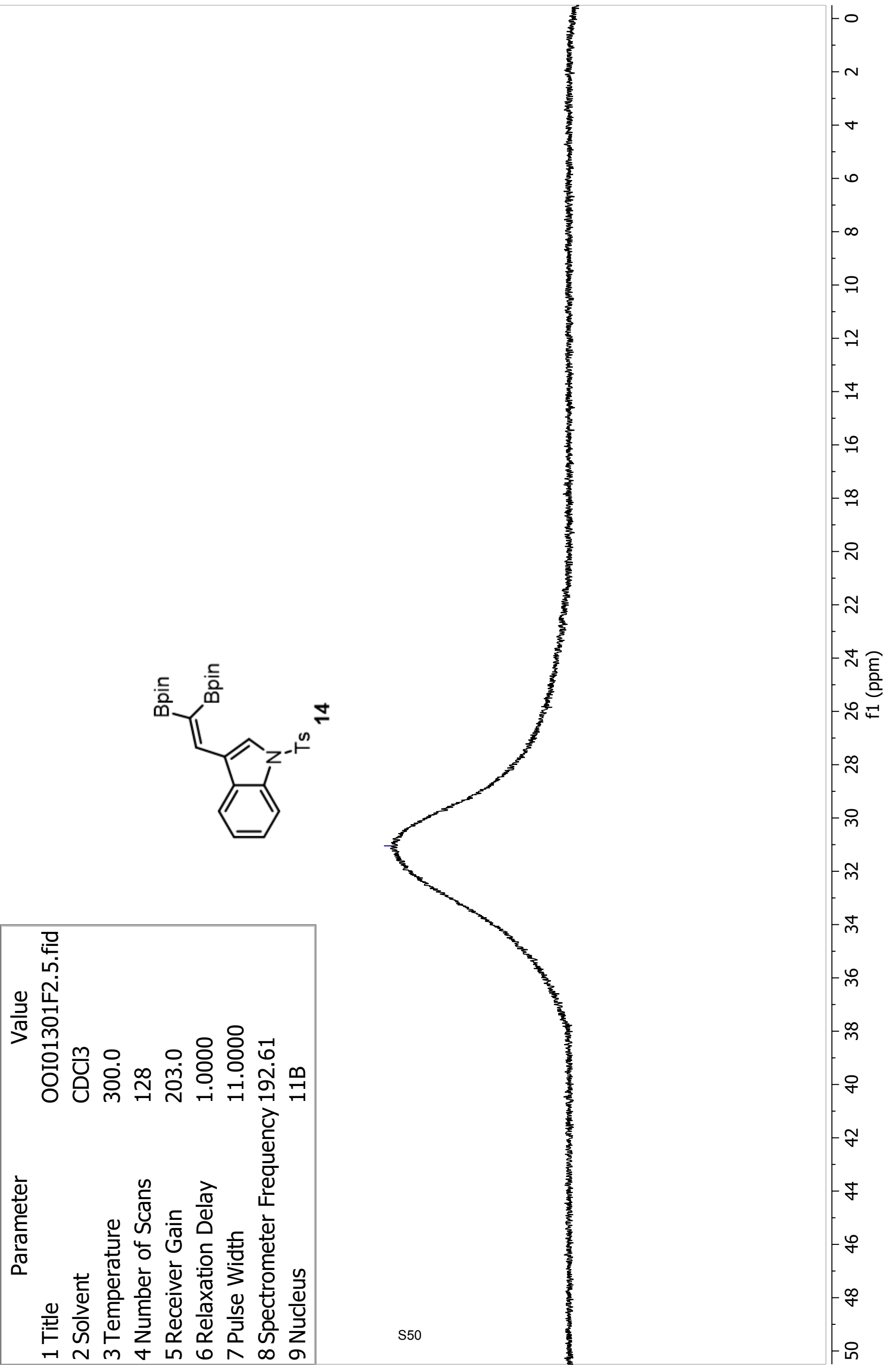

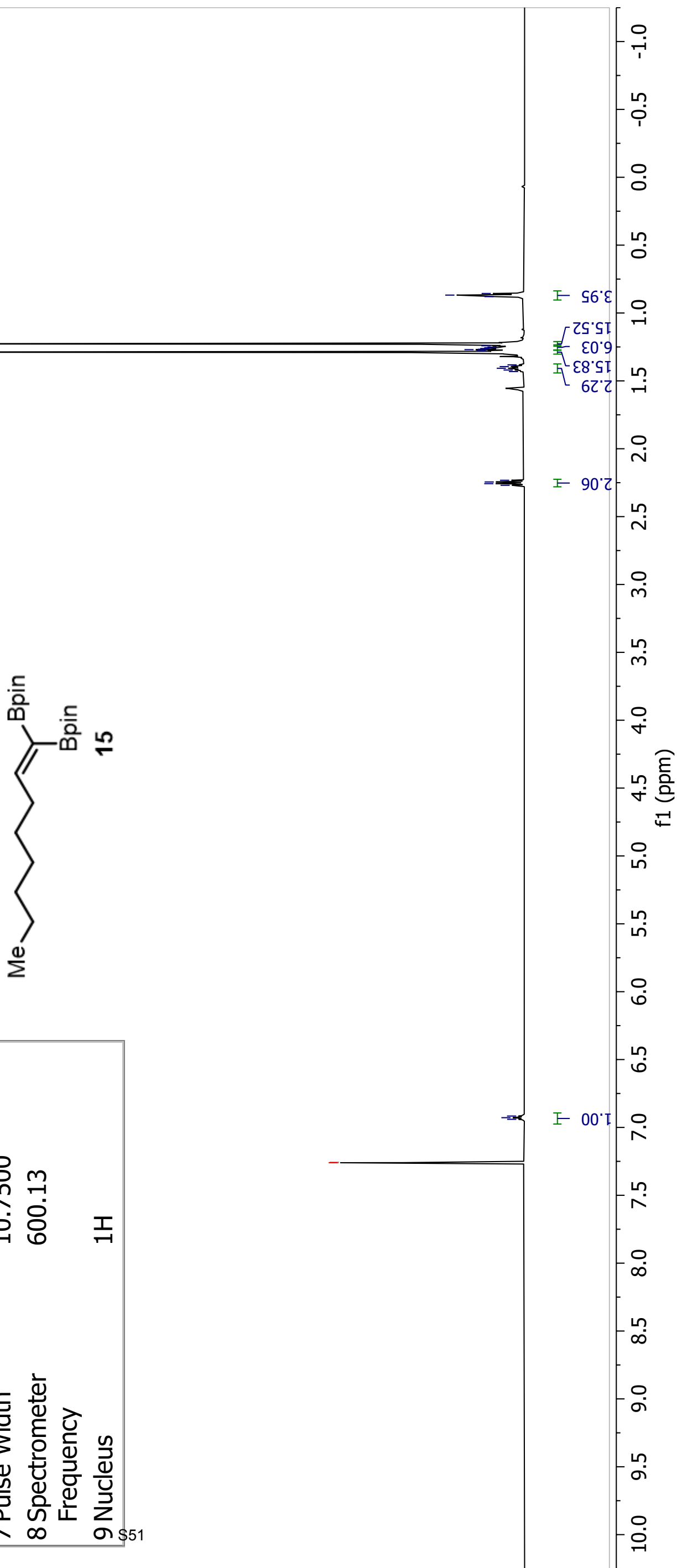
$\varepsilon \tau^{*} \downarrow I-$

69.2 ح

$\left.\angle 8^{\circ}+2\right\rangle$

$96^{\circ} \circ z^{\prime}$

हโ'6乙ד

8I'6乙

Z8. IE

$8 \varsigma^{\circ} \mathrm{S} \varepsilon^{\prime}$
عاده ૬ $6^{\circ} 9 L$

हागव 9I' $\angle L$

हाગ $9 I^{\prime} \angle L$

हागهગ $\angle \varepsilon^{\prime \prime} \angle L$

$26.28>$

乙乙' $\varepsilon 8^{\prime}$

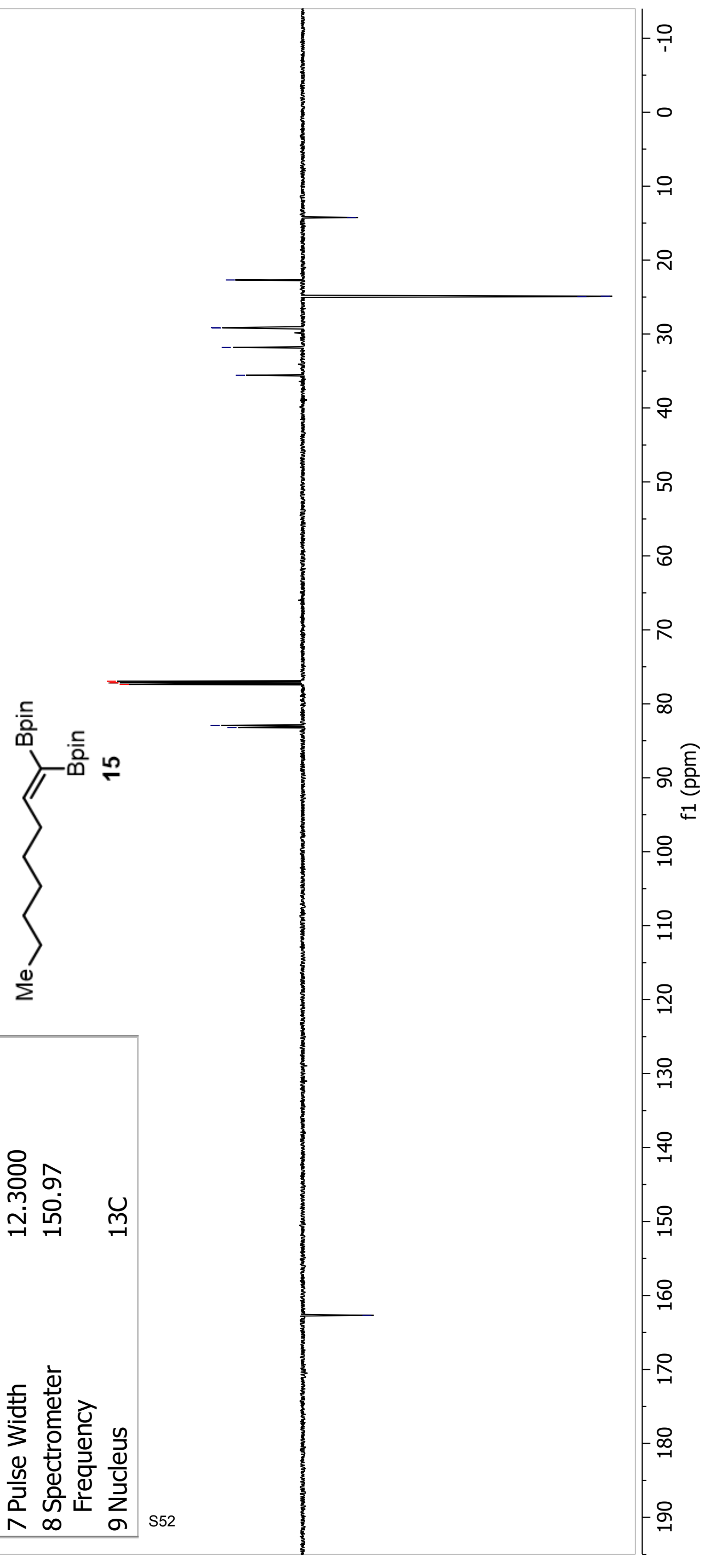



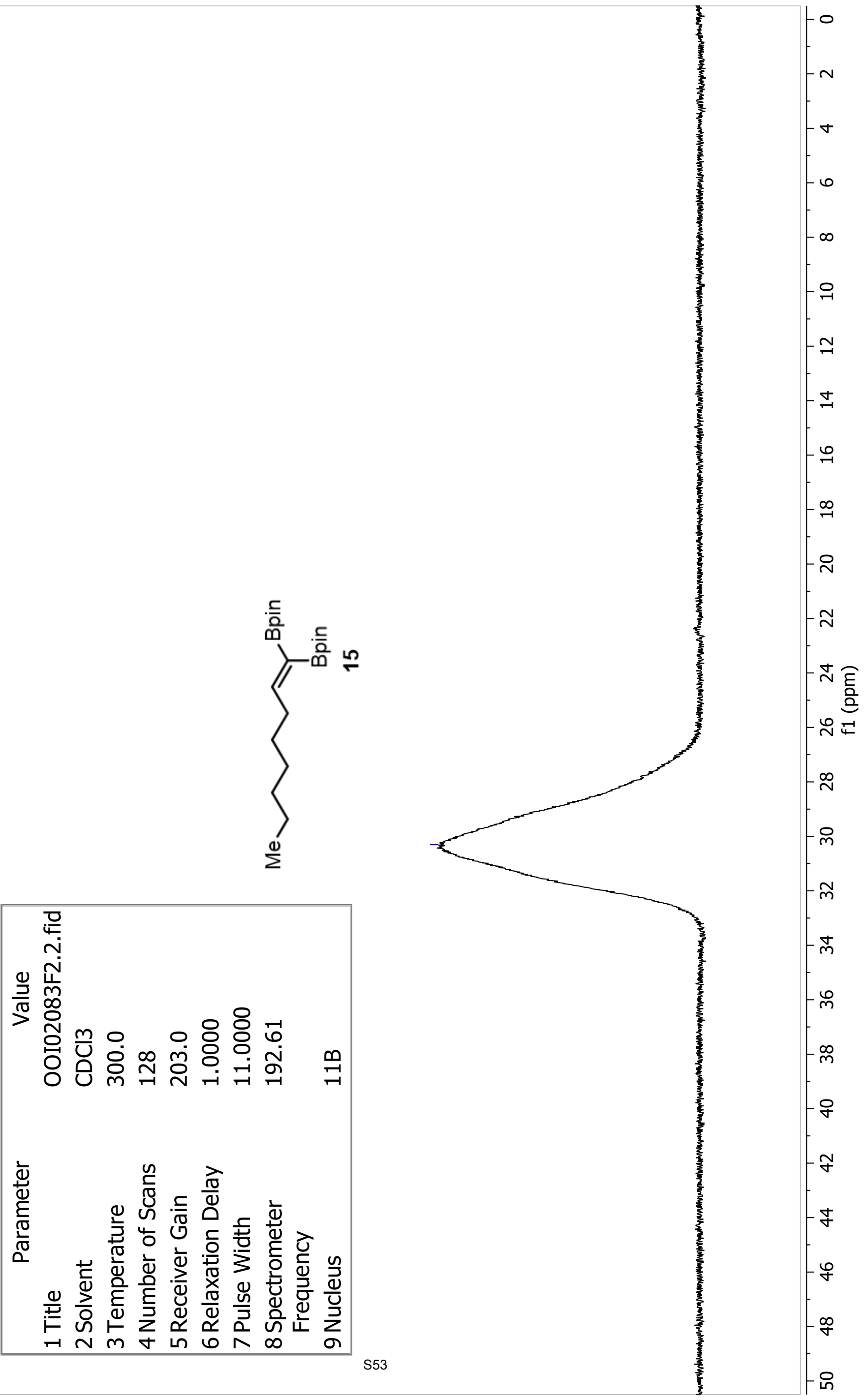

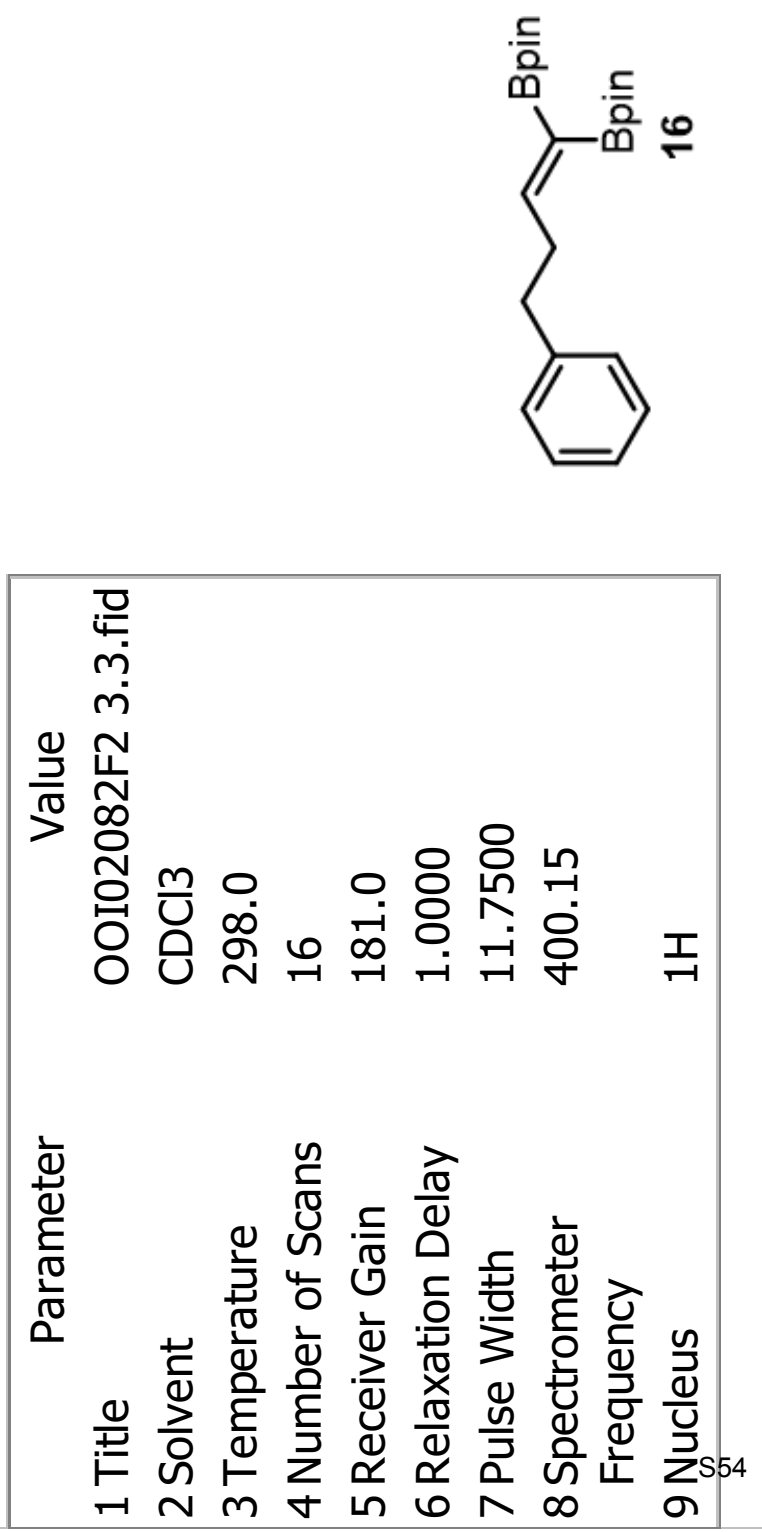
$\left.\begin{array}{l}16 \circ z \\ 56 \circ z\end{array}\right\rangle$

$\varepsilon L ' S \varepsilon-$

$6 t^{\circ} \angle \varepsilon-$


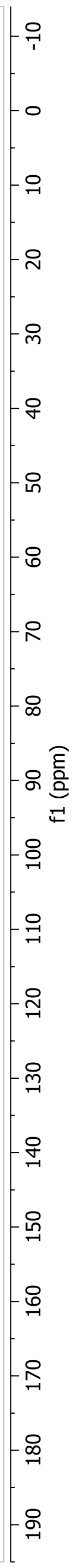




$\left.\varepsilon \mathcal{S}^{\prime} \varepsilon\right]$

\begin{tabular}{l}
$\dagger \varsigma^{\prime} \varepsilon$ \\
$\varsigma^{\prime} \varepsilon$ \\
\hline
\end{tabular}

$\angle 8^{\circ} 9$

$68.9 \frac{1}{5}$

06.9

हाગ๐ $92^{\circ} \angle$ -

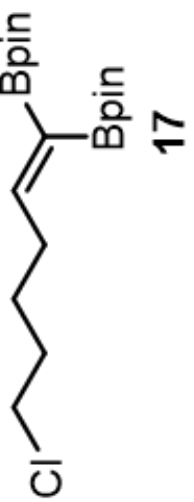




$06 \circ \mathrm{b}$

$96 . b 2 \frac{1}{02.92}$

0 ' $Z \varepsilon-$

It $\sqcup \varepsilon-$

$00^{\circ} \varsigma b-$

हाગव ऽ6.9L




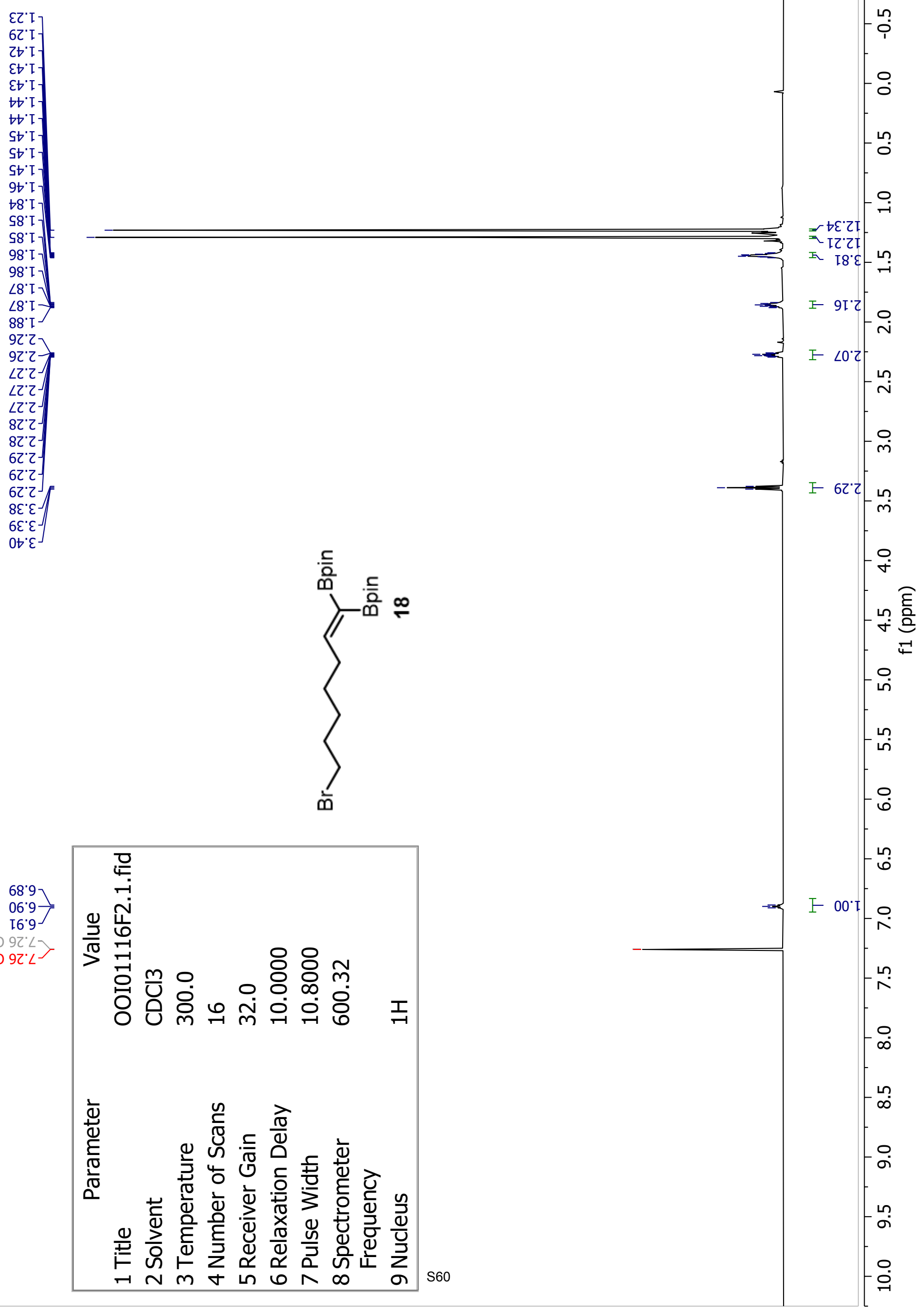







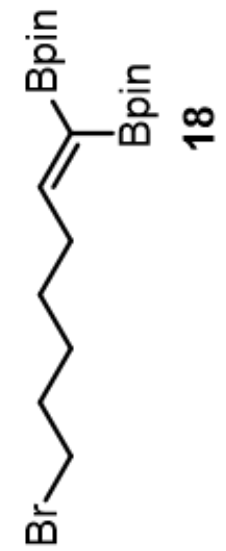

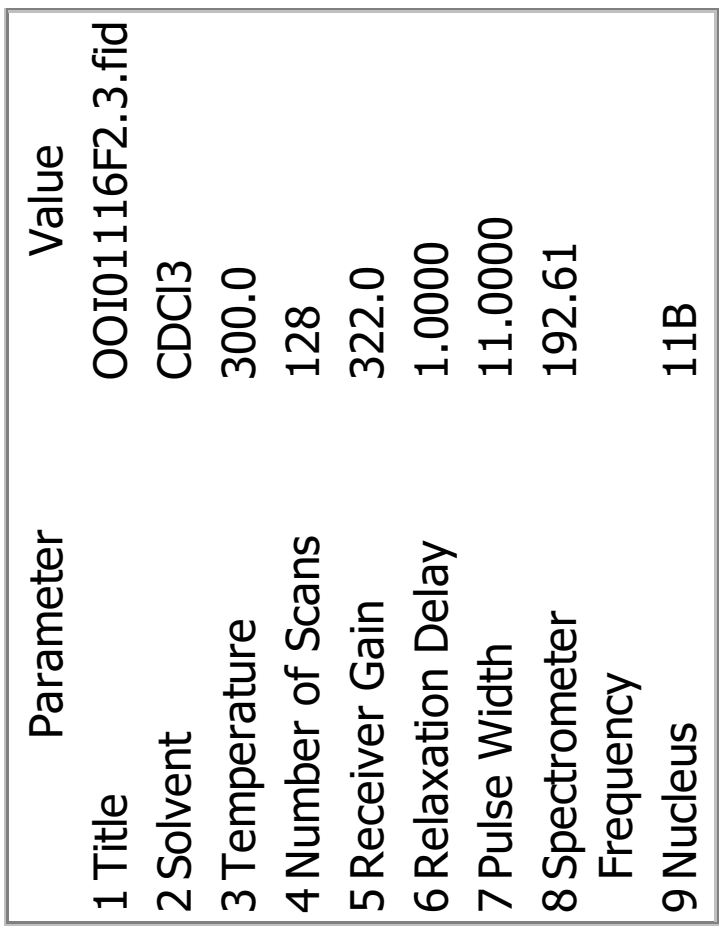

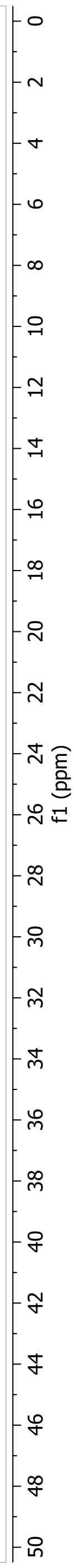



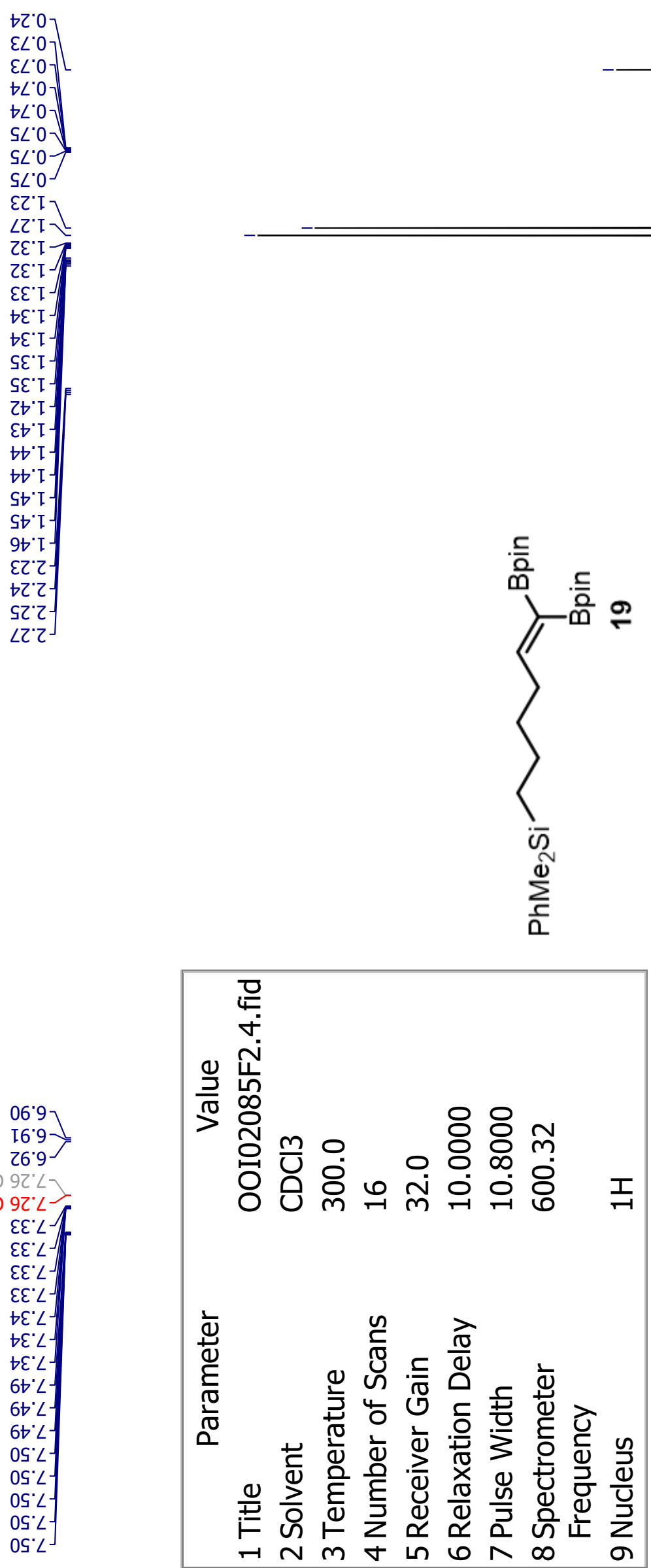
Z9'SI-

98' $\varepsilon 乙 7$

$88^{\circ} \circ \tau \succ$



$8 乙^{\circ} \varsigma \varepsilon-$

عાગه ५6.9L

हागه 9.' $\angle L]$

हाग 9T. $\angle L]$

โ6.28


0乙'ย8

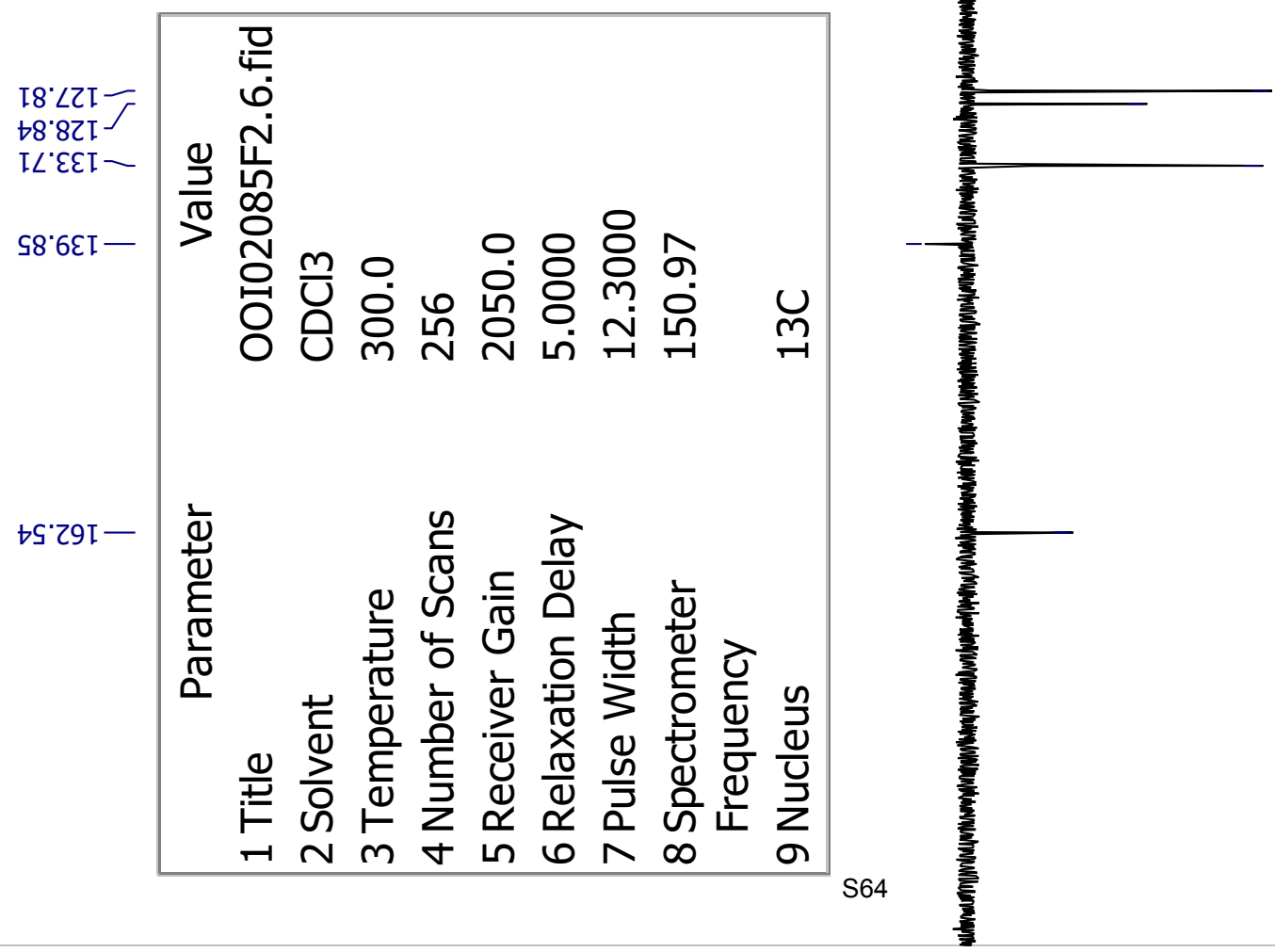




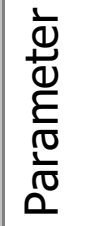

ข

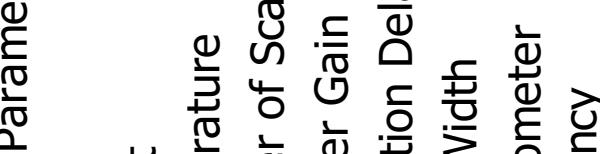

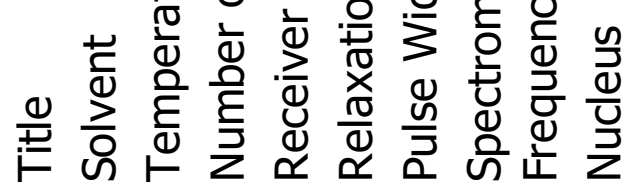






II'ย--

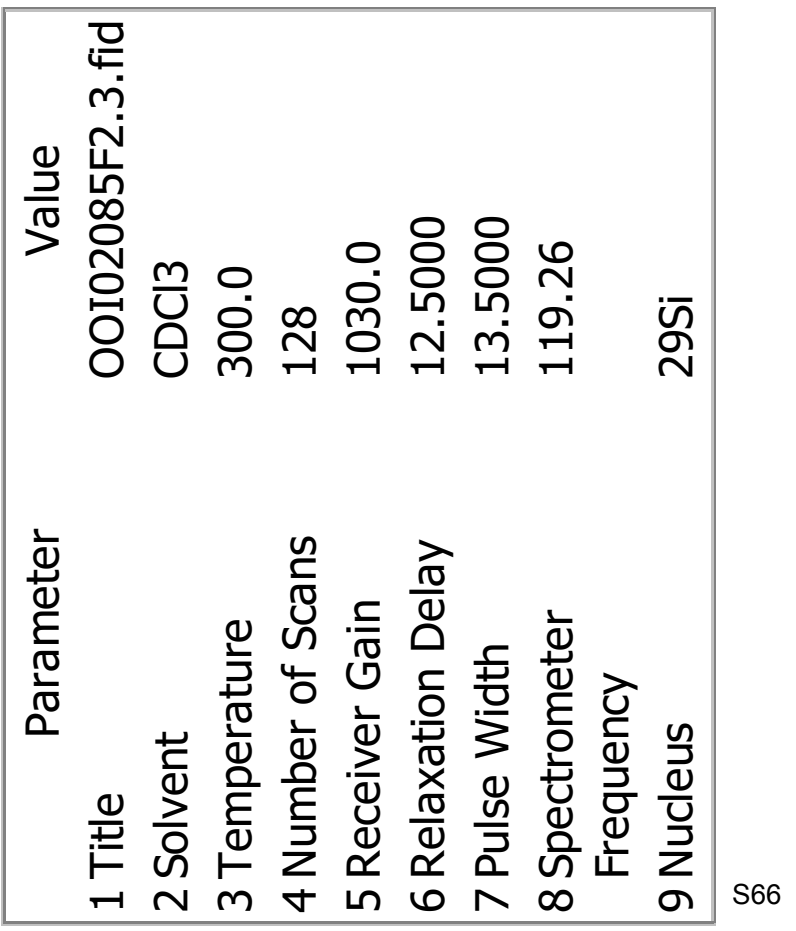







$60^{\circ}+1-$

ยร'6I-

$88^{\circ} \mathrm{tz}$

$96 \circ z$

60.925

$\angle 0^{\circ} 62-5$

$8 \angle 26 \mathrm{Z}^{\circ}$

เ0'حह

โS' $\subseteq \varepsilon$

$08^{\circ} 0<>$

$86^{\circ} 0 L^{\prime}$

हાગه $56^{\circ} 9 \angle>$

हाग๐ 9. $\angle L \frac{\searrow}{T}$

हाગه 9T" $\angle L J]$

हાગ $\angle \varepsilon^{\prime} \angle L$

$\rightarrow 6.28-$

$\rightarrow Z^{\prime} \varepsilon 8$
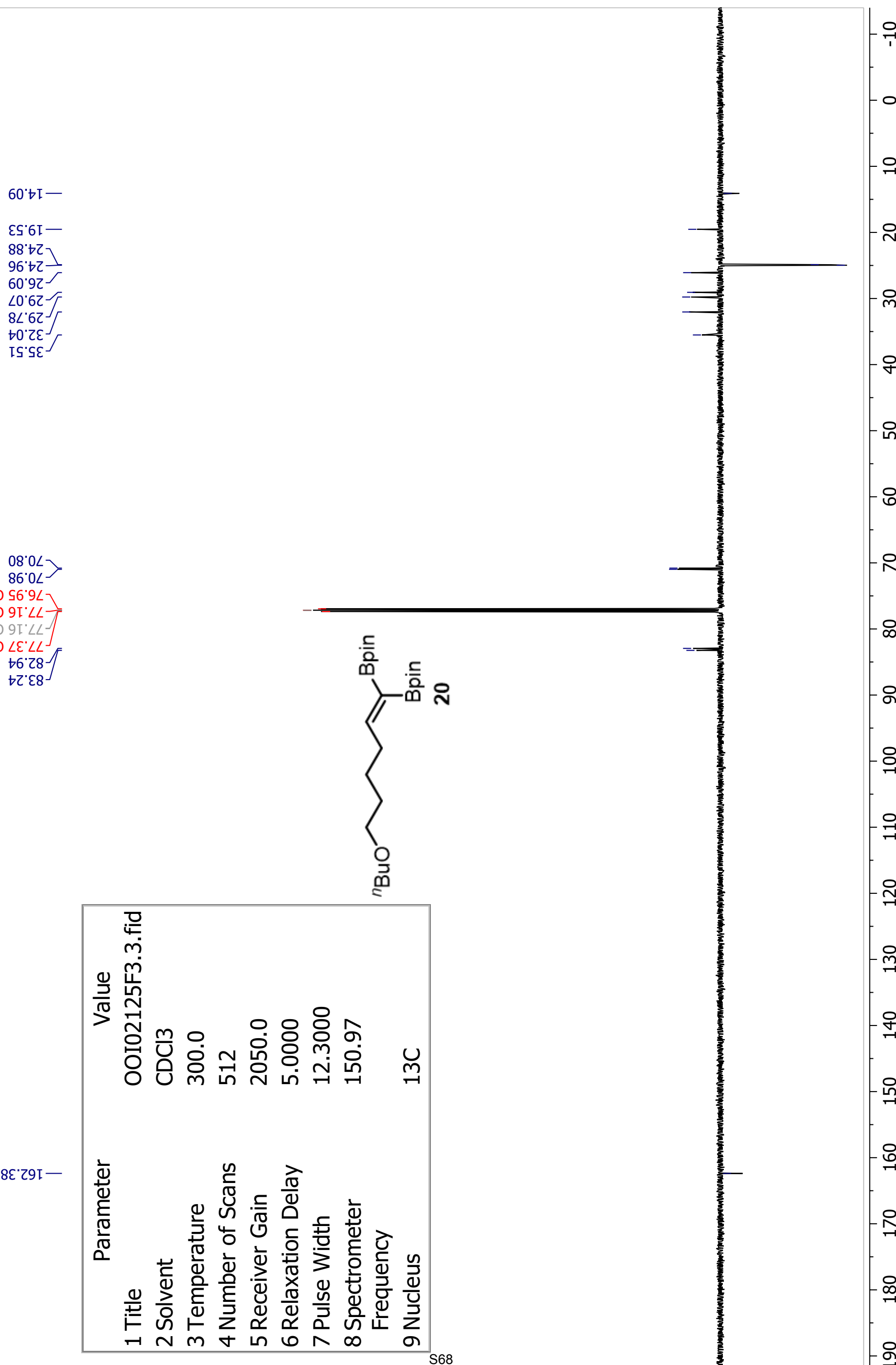

응 을

욱

윽

ํำ

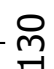

욱

눈

8

욕

$\stackrel{\infty}{-\infty}$ 

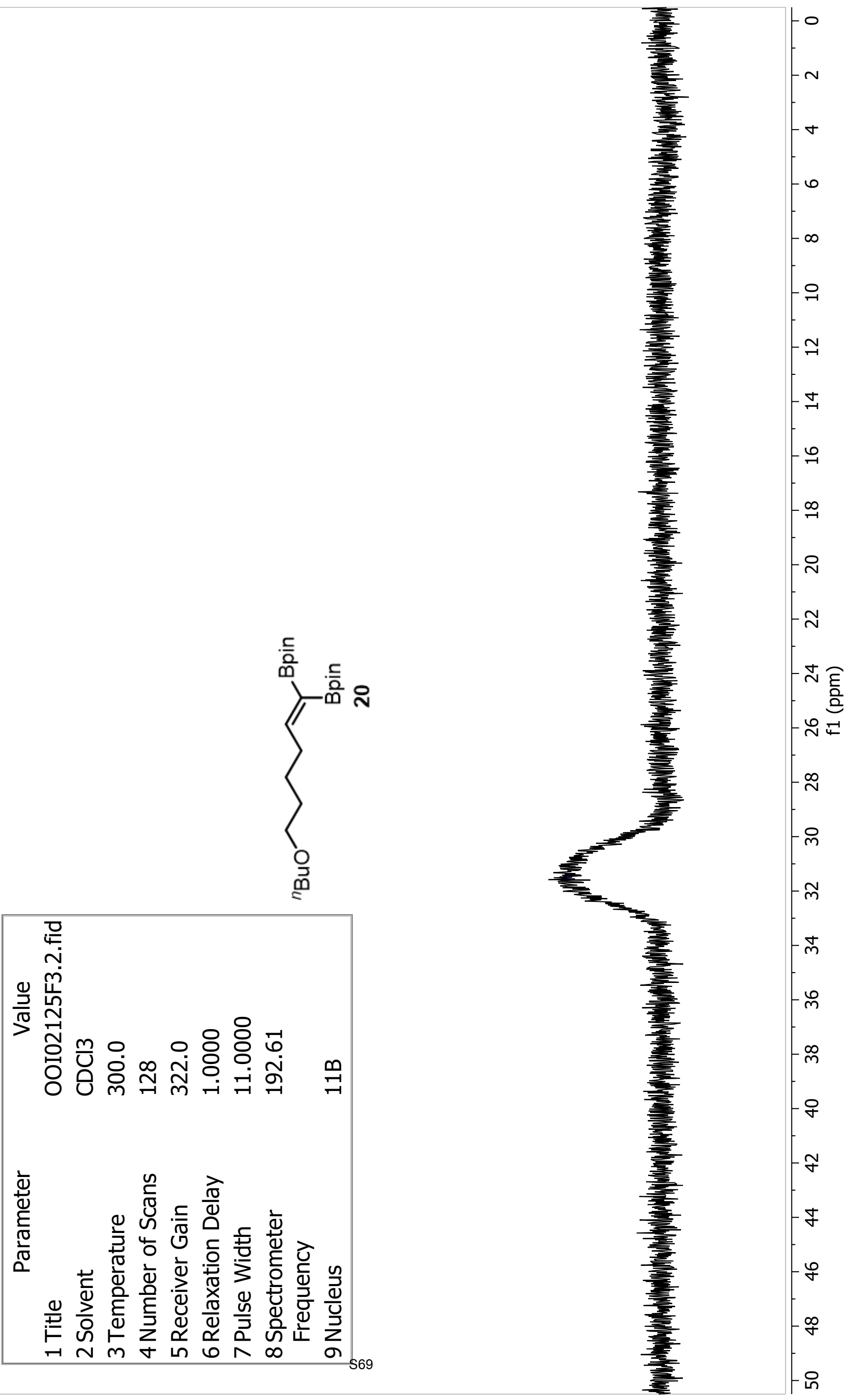


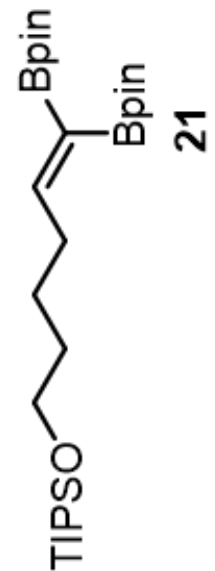

6.9
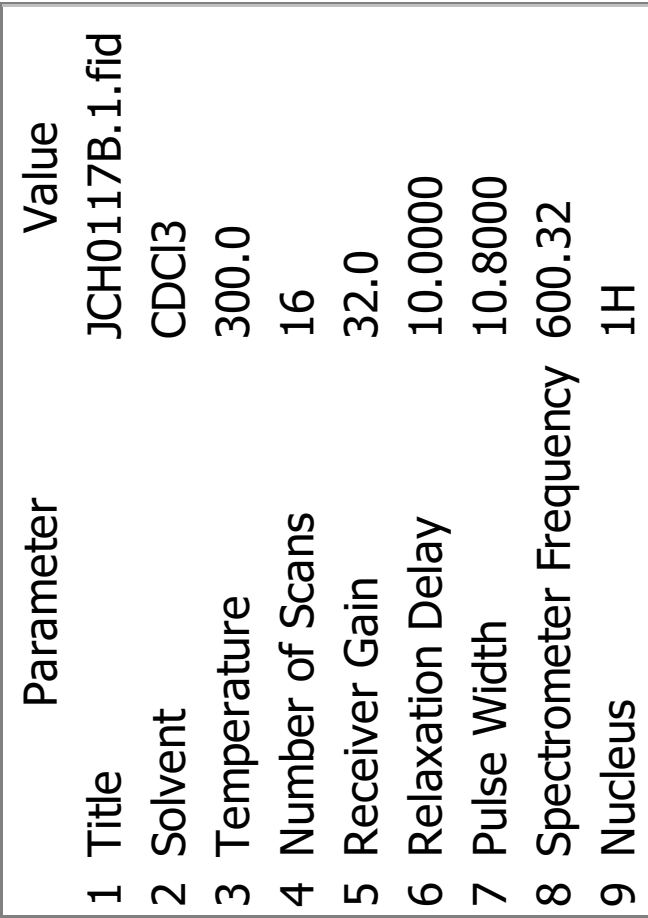

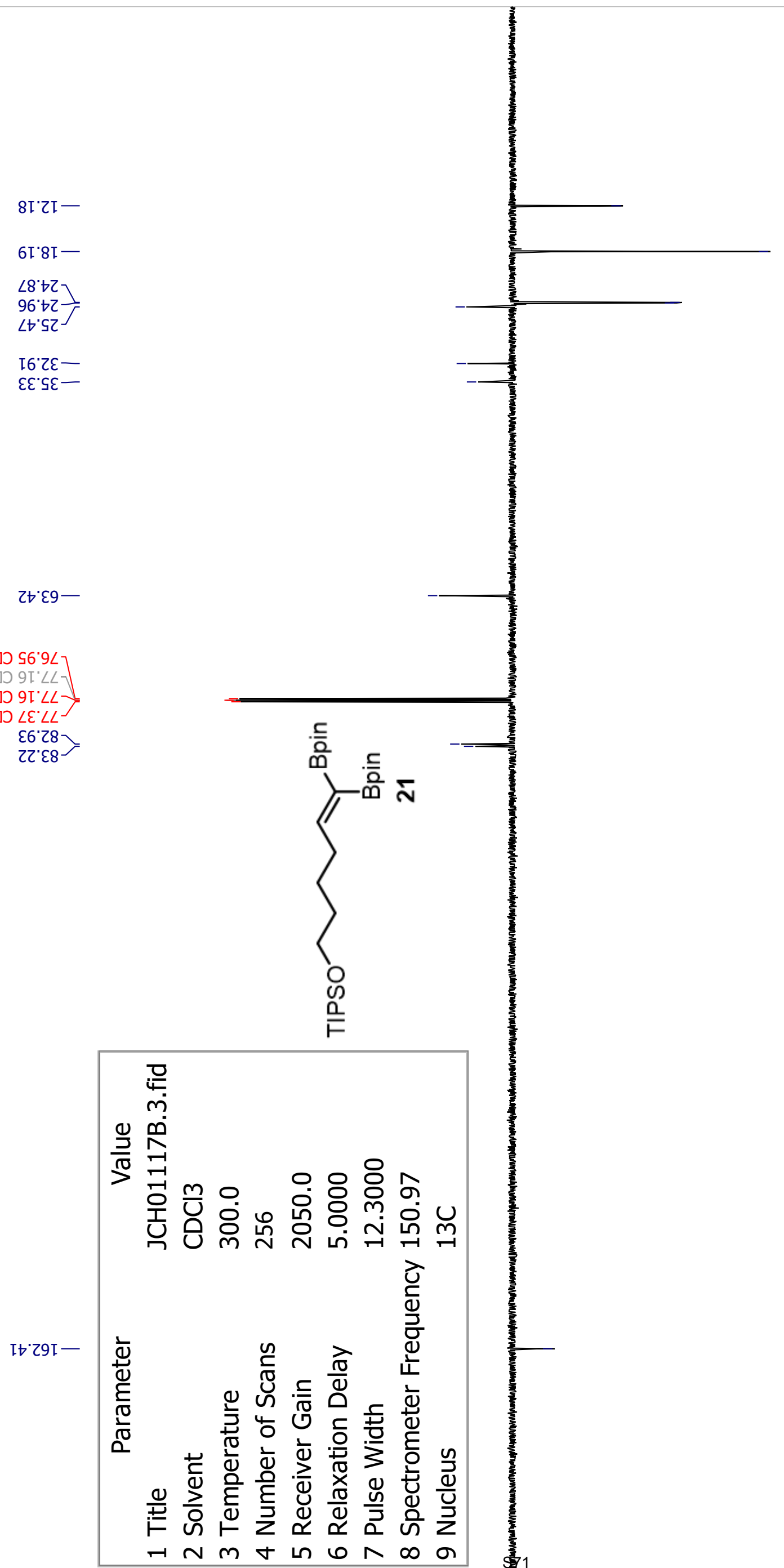


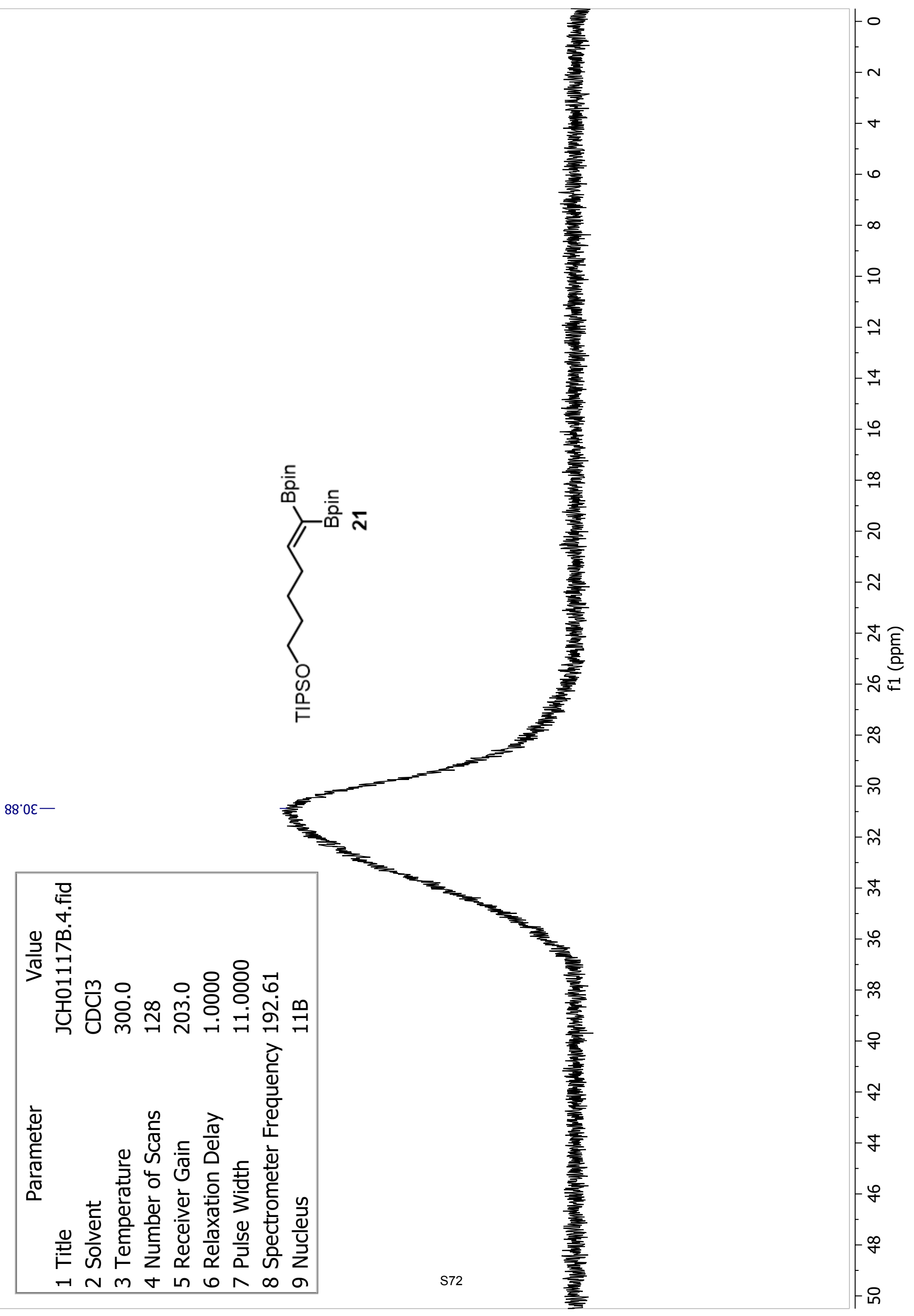



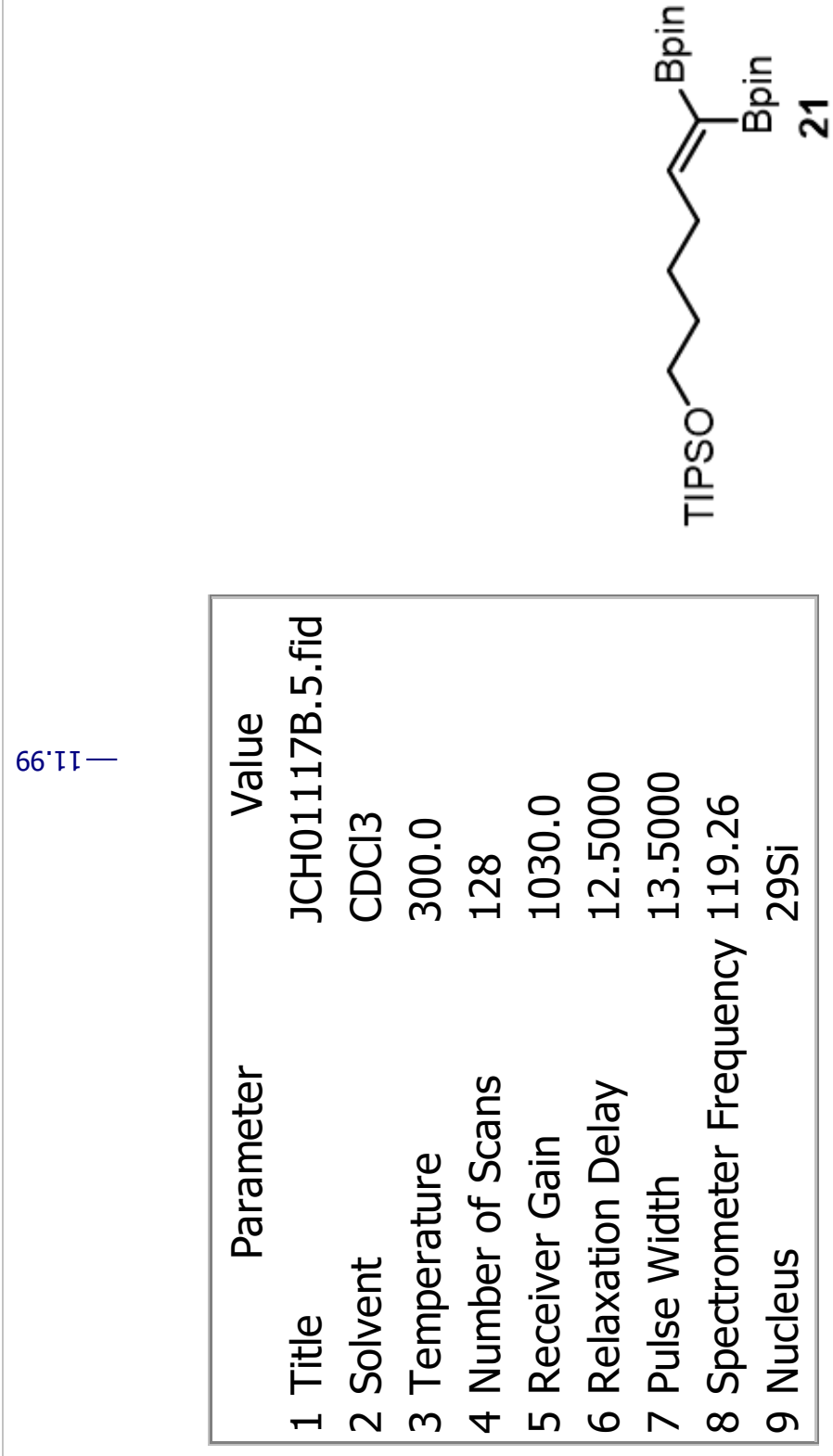


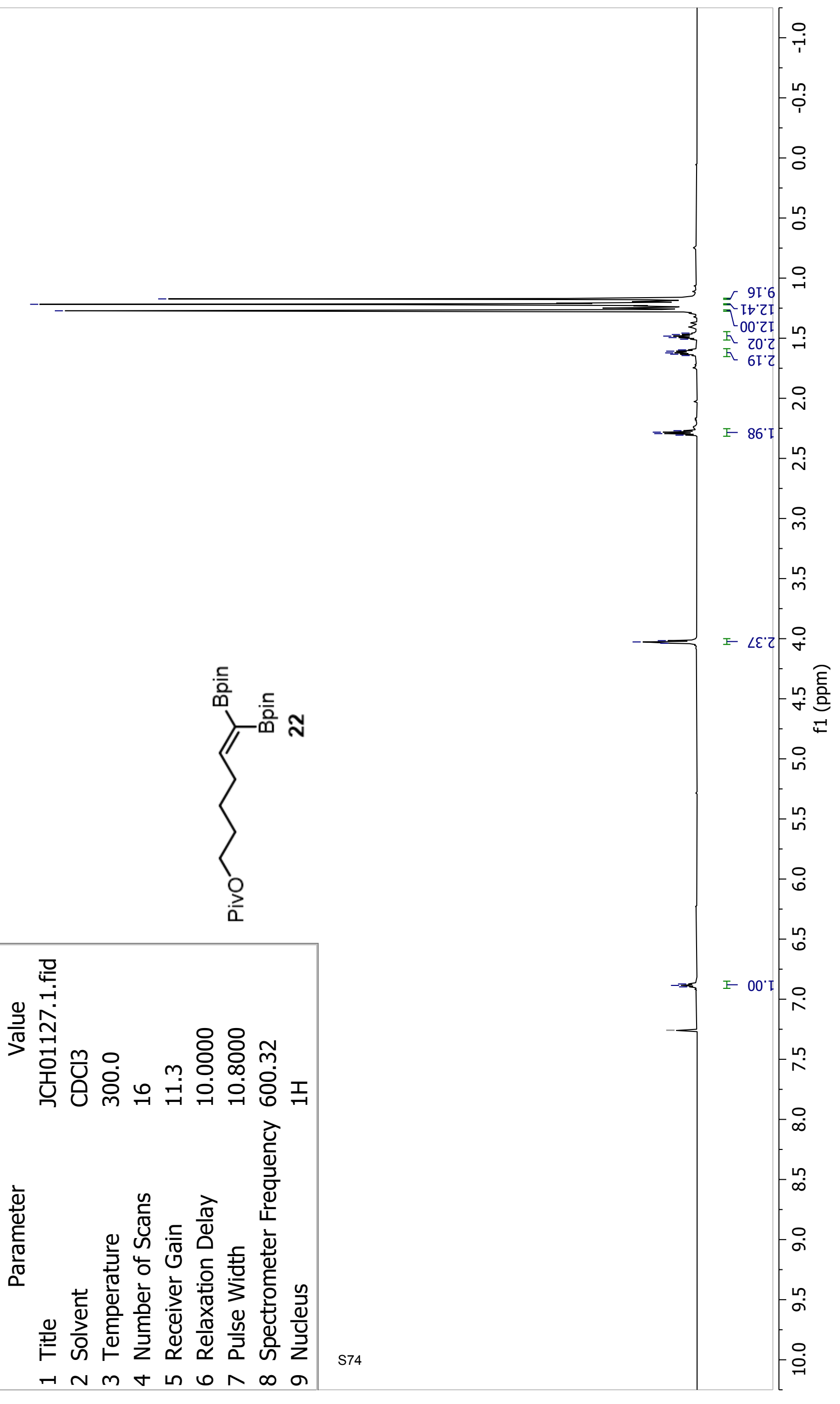

$\angle 8^{\circ} 9$ $68^{\circ}$ 06.9

हाวดว $92^{\circ} L$ 
$\angle 8^{\circ} \circ 2$

$\rightarrow 6 \circ 2$

$\angle b \cdot S Z$

$\angle \varepsilon^{\circ} \angle Z^{-}$

$6 \varepsilon^{\prime} 8 \tau^{\circ}$

$26^{\circ} \triangleright \varepsilon-$

$98.8 \varepsilon-$

$\varsigma \varepsilon^{\circ} \downarrow 9-$

\section{عاده $56^{\circ} 9 L$}

हाગव 9I' $\angle L$

हाग 9T' $\angle L J$

00'ع8

$87^{\prime} \varepsilon 8$



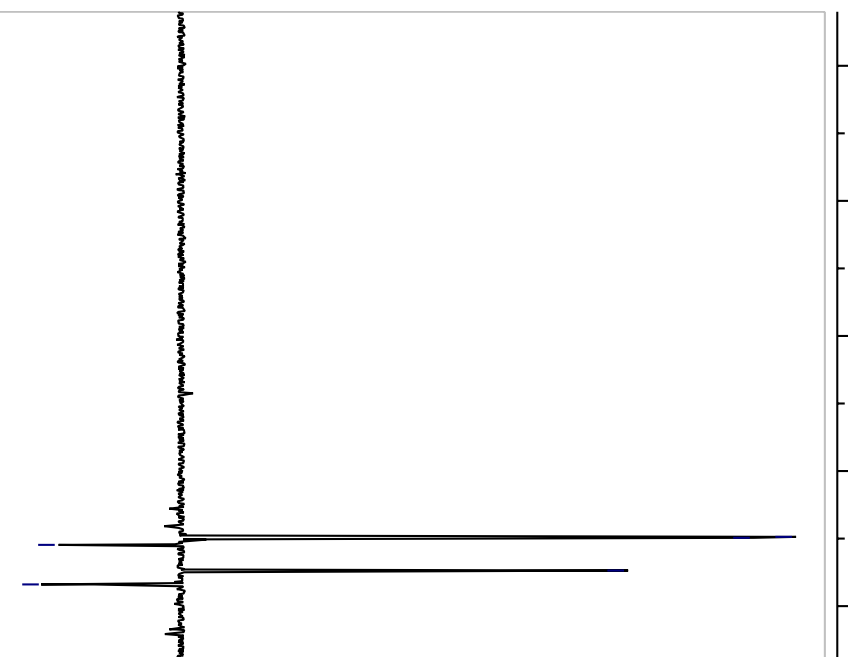




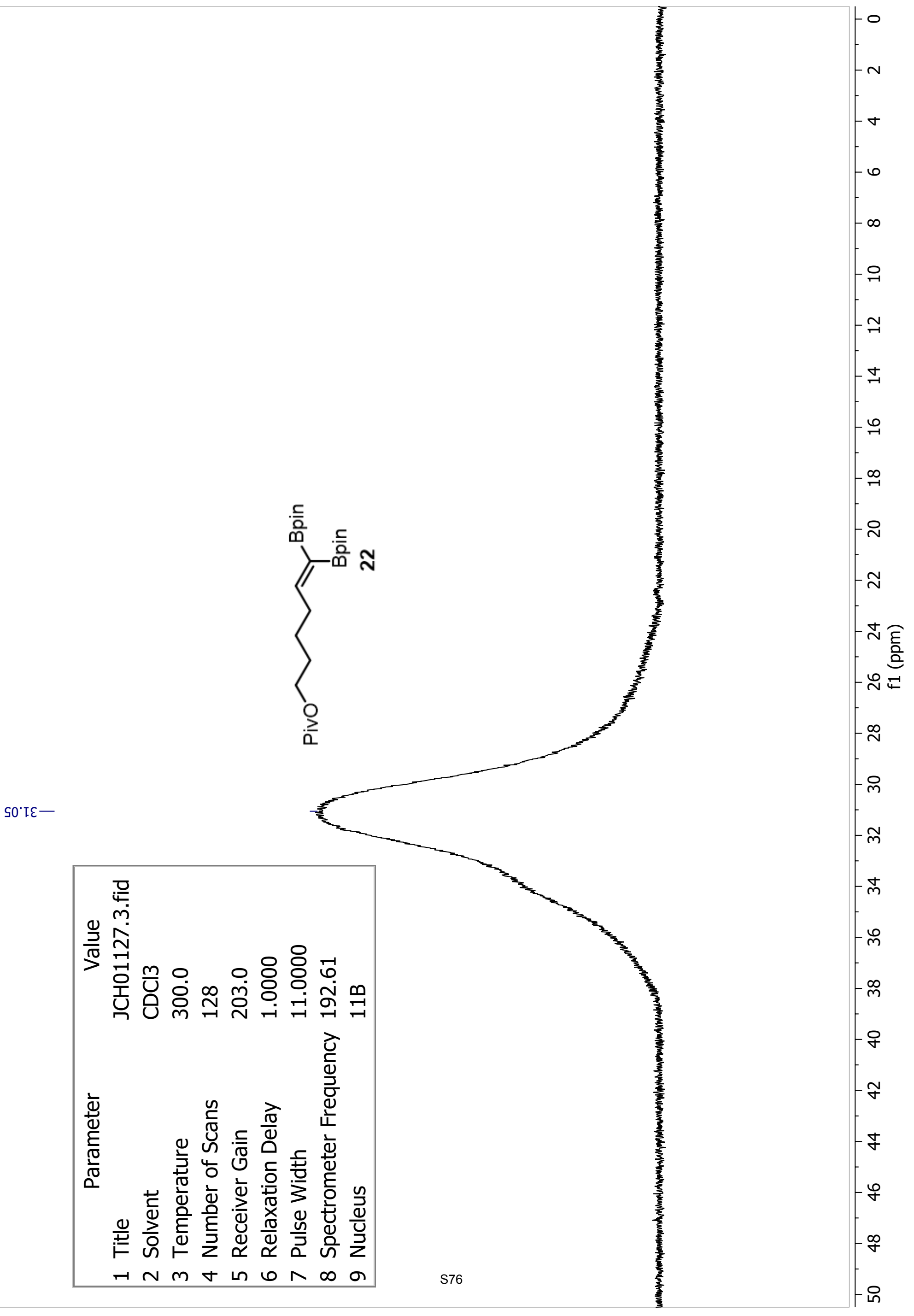


26.0

EZ' $T$

tट.1

$8 \mathrm{C}^{\circ} \mathrm{I}-$

$6 \mathrm{C}^{\circ} \mathrm{I}$

OE' I

9I' 2

$8 \mathrm{I}^{\prime} \mathrm{\gamma}$




$88^{\circ} \downarrow \tau$
$66^{\circ} \triangleright \tau$

$\downarrow L ' 6 Z=$

$0 t^{\circ} T \varepsilon-$

$\varepsilon L^{\prime} 6 t-$

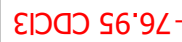

हागه 9I" $\angle L$

हाગ $9 I^{\prime} \angle L$

हाग๐ว $\angle \varepsilon^{\prime} \angle L$

$26.28>$

$9 乙^{\prime} \varepsilon 8^{\prime}$
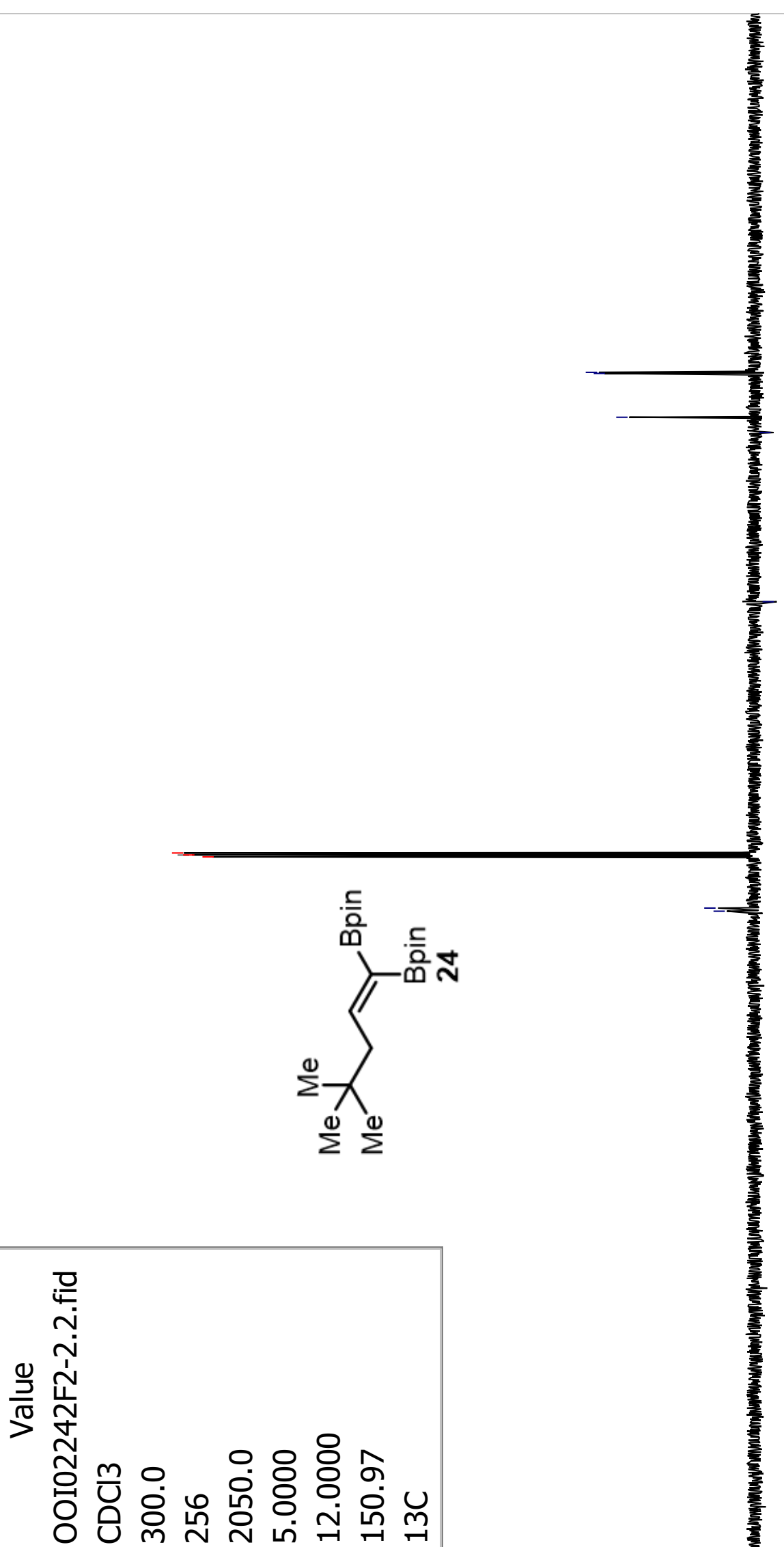

$\frac{\frac{1}{0}}{\frac{1}{0}}$ 


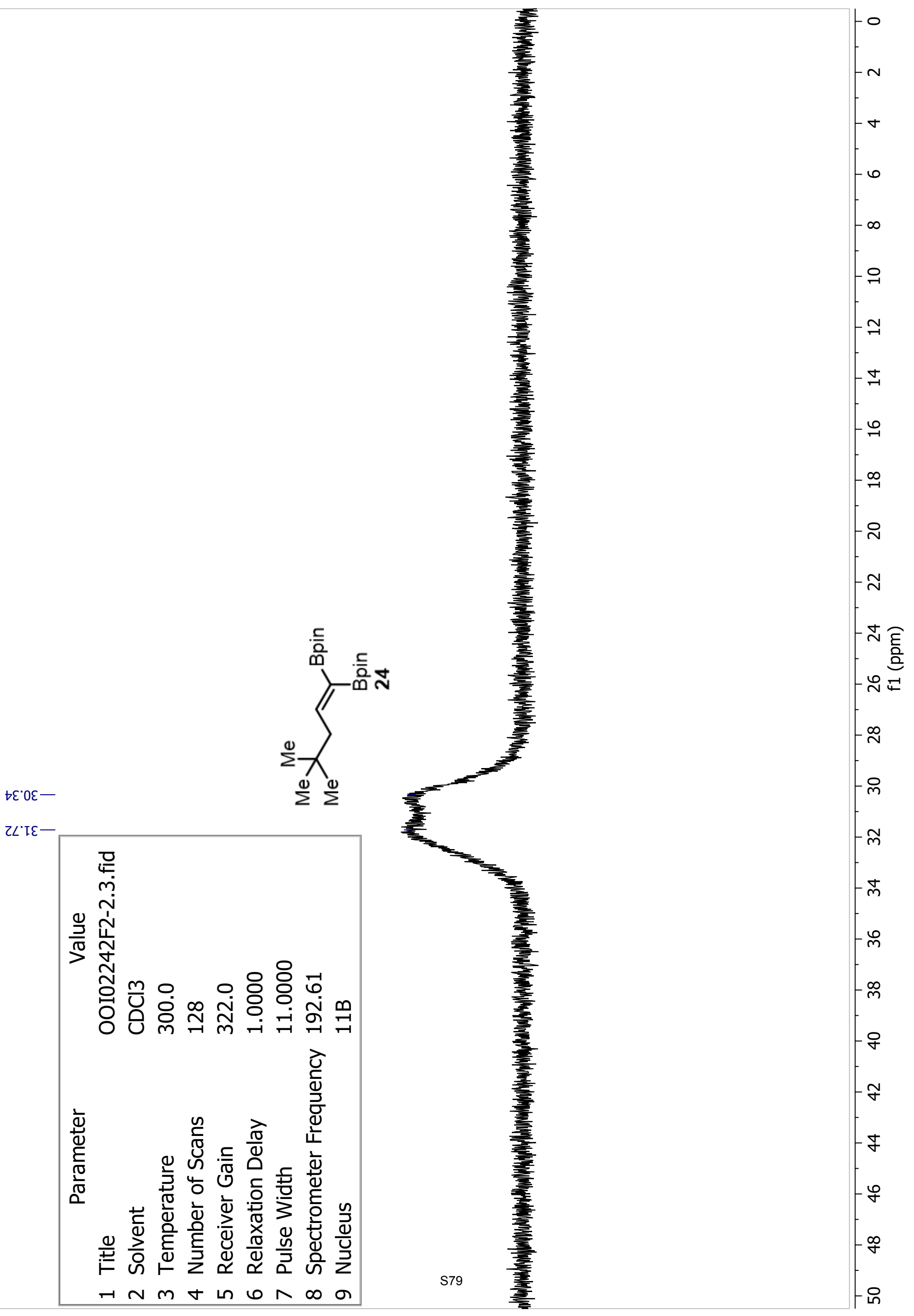




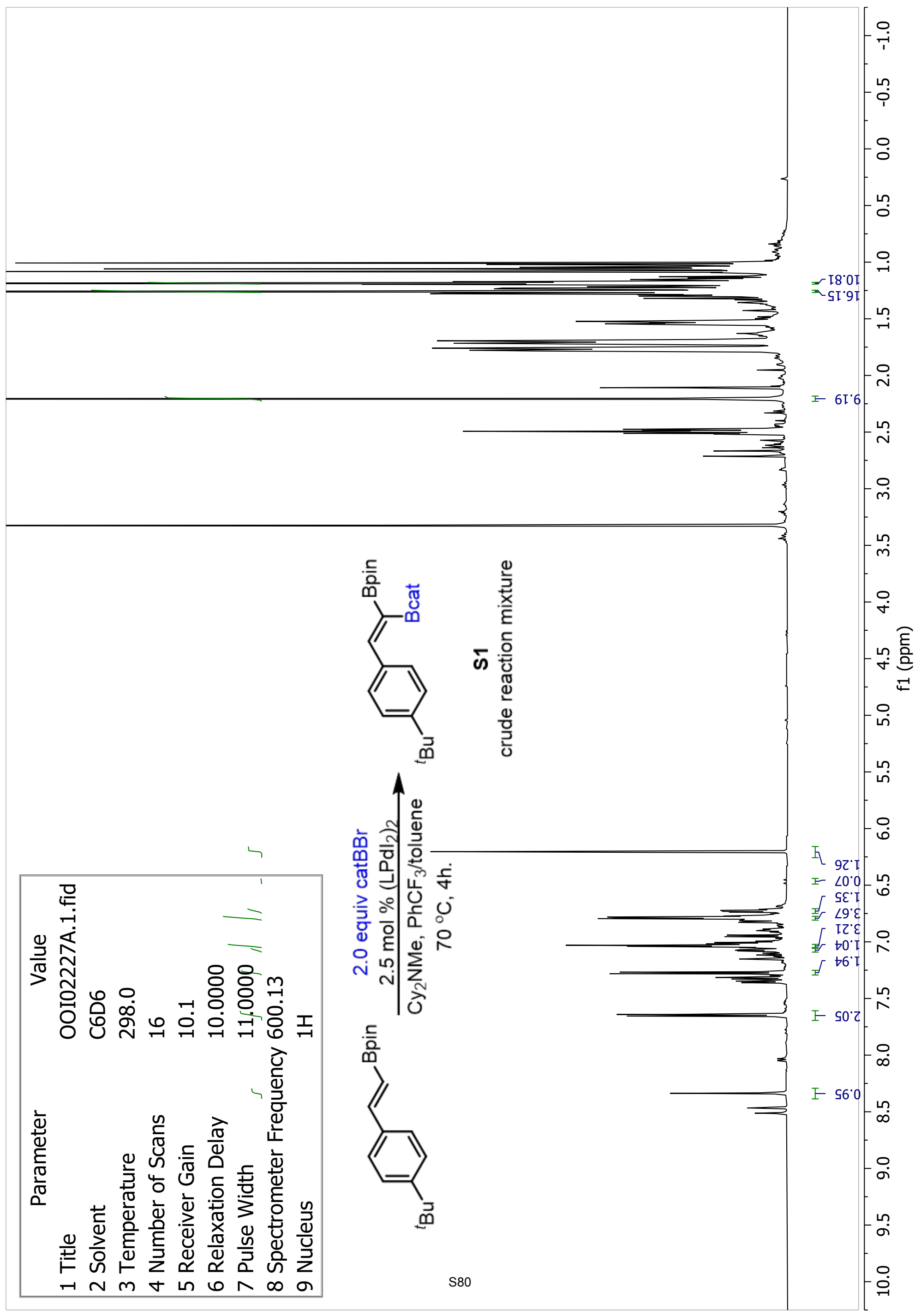

